\title{
Catalytic Enantioselective Ring-Opening Reactions of Cyclopropanes
}

\author{
Vincent Pirenne, ${ }^{\dagger}$ Bastian Muriel ${ }^{\dagger}$ and Jerome Waser* \\ †These authors contributed equally to this work. \\ Laboratory of Catalysis and Organic Synthesis, Institute of Chemical Sciences and Engineering, Ecole Polytechnique \\ Fédérale de Lausanne, EPFL SB ISIC LCSO, BCH 4306, 1015 Lausanne, Switzerland.
}

\begin{abstract}
This review describes the development of enantioselective methods for the ringopening of cyclopropanes. Both approaches based on reaction of non-chiral cyclopropanes and (dynamic) kinetic resolutions and asymmetric transformations of chiral substrates are presented. The review is organized according to substrate classes, starting by the more mature field of DonorAcceptor cyclopropanes. Emerging methods for enantioselective ring-opening of Acceptor- or Donor-only cyclopropanes are then presented. The last part of the review describes the ringopening of more reactive three-membered rings substituted with unsaturations, with a particular focus on vinylcyclopropanes, alkylidenecyclopropanes and vinylidenecyclopropanes. In the last two decades, the field has grown from a proof of concept stage to a broad range of methods for accessing enantio-enriched building blocks, and further extensive developments can be expected in the future.
\end{abstract}

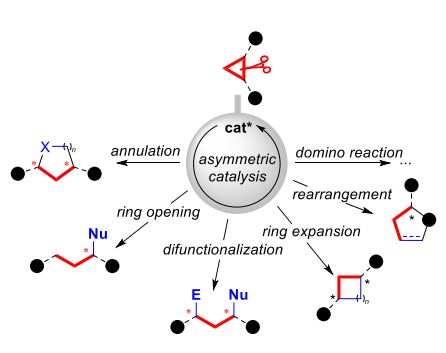

\section{Contents}

1. INTRODUCTION 1

2. DONOR-ACCEPTOR CYCLOPROPANES …............... 2

2.1. ANNULATION REACTIONS .................................. 2

2.1.1. (3+2) Annulations ................................... 2

2.1.2. (3+3) Annulations ................................. 6

2.1.3. Other annulations..................................... 8

2.2. RING-OPENING REACTIONS.................................. 9

2.2.1. Addition of heteroatom nucleophiles ........ 9

2.2.2. Friedel-Crafts alkylations .......................... 10

2.2.3. Base-mediated fragmentation................. 11

2.3. OTHER RING-OPENING/CLOSING PROCESSES ........... 12

2.3.1. Cloke-Wilson rearrangement................... 12

2.3.2. $\quad$ Ring opening/cyclization with primary

amines .......................................................... 12

2.3.3. Via enamine catalysis ............................ 13

2.3.4. Other.............................................. 13

3. ACCEPTOR-ACTIVATED CYCLOPROPANES ......14

3.1. ANNULATION REACTIONS .................................. 14

3.2. RING-OPENING REACTIONS.............................. 16

3.2.1. Via nucleophile/base catalysis................. 16

3.2.2. Via enamine catalysis ............................. 16

4. DONOR-ACTIVATED CYCLOPROPANES................17

5. VINYLCYCLOPROPANES_.......................................18

5.1. $(3+2)$ ANNULATIONS....................................... 18

5.1.1. Palladium catalysis ................................ 19
5.1.2. Synergistic palladium-amine catalysis..... 24

5.1.3. Rhodium catalysis................................ 25

5.1.4. Thiyl radical catalysis............................. 26

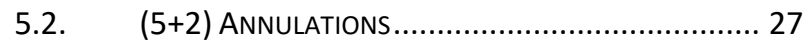

5.3. (4+3) ANNULATIONS...................................... 28

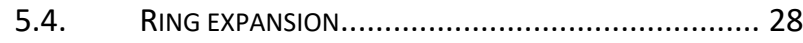

5.5. RING-OPENING REACTIONS............................. 29

6. ALKYLIDENECYCLOPROPANES..............................30

6.1. ANNULATION REACTIONS.................................. 30

6.2. RING EXPANSIONS ...................................... 31

6.3. RING-OPENING / DIFUNCTIONALIZATION................ 33

7. VINYLIDENECYCLOPROPANES .............................34

8. CONCLUSION AND OUTLOOK .................................36

\section{Introduction}

Cyclopropanes, as smallest carbocycles, have always attracted the attention of chemists. The presence of tortional and angle strain for a total of about $115 \mathrm{KJ} \mathrm{mol}^{-1}$ sets the stage for ringopening reactions to access functionalized chiral building blocks. ${ }^{1}$ Even with the presence of ring-strain, the carboncarbon bonds remain kinetically stable, so that catalysts were developed to achieve ring-opening under mild conditions. This synthetic strategy is attractive, as many methods are now available for accessing cyclopropanes stereoselectively. Therefore, the most common approach for accessing enantioenriched building blocks from cyclopropanes is through their stereospecific (enantio- and/or diastereospecific) ringopening. ${ }^{2}$ An alternative approach is the enantioselective ring- 
opening of cyclopropanes, which has been extensively investigated since 2005, and is having therefore an increasing impact in synthetic chemistry. Nevertheless, to the best of our knowledge, this topic has never been described in a single dedicated review. Only the specific case of enantioselective ring-opening of Donor-Acceptor cyclopropanes has been recently covered. ${ }^{3}$

From the point of view of enantioinduction, two strategies can be followed (Figure 1A):

1) Starting from non-chiral cyclopropane (Class I), either simple substrates with substituents on a single carbon or more complex meso-compounds.

2) Using more complex multi-substituted cyclopropanes, which are themselves chiral (Class II). In this case, kinetic resolutions or asymmetric transformations can be used to access enantiopure ring-opening products starting from racemic cyclopropanes. Nevertheless, the theoretical maximal yield of such transformations is only 50\%. Major efforts have been consequently invested in the last decades to develop dynamic processes, allowing to convert the starting material completely to the desired product.

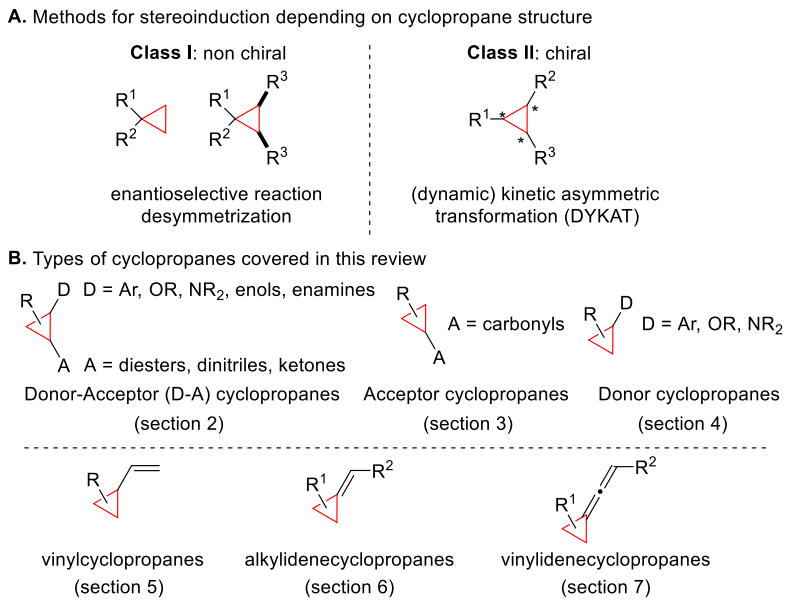

Figure 1. Methods for stereoinduction (A) and types of cyclopropanes covered in this review $(B)$.

The choice of chiral catalyst and activation principle is highly dependent on the structure of the cyclopropanes. ${ }^{4}$ Therefore, this review has been organized along the most important classes of three-membered rings (Figure 1B). It will start with DonorAcceptor (D-A) cyclopropanes (section 2). The inherent pushpull system in vicinal D-A cyclopropanes polarizes the C-C bond and strongly facilitates the ring-opening process. Upon activation, a formal 1,3-zwitterion is formed and can react with a nucleophile, an electrophile or a multiple bond system leading to acyclic or cyclic products. This exceptional reactivity has been widely used in synthetic chemistry and has been covered by numerous reviews, ${ }^{5-16}$ with only one dedicated to enantioselective transformations. ${ }^{3}$ The most frequently used acceptor is by far a diester group, although ketones, nitriles and nitro groups have been used in some instance. The donors are most frequently (hetero)aryls and heteroatoms, with some examples of enolates or enamines. Alkyl groups are only weakly electron-donating and have not been considered as donors for this review. Section 3 and 4 will be dedicated to Acceptor- and Donor-only-activated cyclopropanes respectively. These classes of substrates are more difficult to activate and have been less investigated, but recent exciting progress has been realized, especially based on transition metal catalysis $^{17-23}$ or the formation of reactive intermediates, such as radicals ${ }^{24}$ or carbocations. Enantioselective methods have just started to emerge in this case. Again, carbonyl groups were most frequently used as acceptors, whereas heteroatoms dominate as donors. Finally, the last three sections will discuss special classes of cyclopropanes substituted with C-C unsaturations: Vinylcyclopropanes (section 5), alkylidenecyclopropanes (section 6) and vinylidenecyclopropanes (section 7). The presence of the more reactive $\pi$ systems opens the way for other types of transition metal-based activations, which has been described in dedicated reviews, ${ }^{25-36}$ none of them specific to asymmetric transformations however. Cyclopropenes have been only rarely used in asymmetric ring-opening transformations and are not covered in this review. ${ }^{37}$

\section{Donor-Acceptor cyclopropanes}

Due to the presence of a highly polarized bond, the catalytic activation of D-A cyclopropanes is easy and has been extensively investigated. ${ }^{3-16}$ Ring-opening is usually triggered by a LUMO-lowering Lewis acid catalyst through coordination of the electron-withdrawing substituent, very often via chelation to a diester, facilitating the attack by a nucleophile. In contrast, the HOMO-raising approach (activation of the electron-donating group) is less frequent and has been mostly achieved via enamine/enolate formation or Umpolung of carbonyls with a carbene catalyst. In this section, enantioselective reactions using D-A cyclopropanes will be discussed, classified according to the chemical transformation: annulations, ring-opening reactions, other ring-opening/closing processes and rearrangements.

\subsection{Annulation reactions}

D-A cyclopropanes have been widely used in annulation reactions for which enantioselective versions were first developed as compared to ring-opening reactions. Due to the importance of five-membered carbocycles and heterocycles, studies have focused on enantioselective $(3+2)$ annulations with different $\pi$-systems such as silyl enol ethers, carbonyl compounds or imines. Enantioselective $(3+3)$ annulations have also been investigated with nitrones or azomethine imines. Finally, less common annulations, such as $(4+3)$ or $(2+2)$ processes, were also reported.

\subsection{1. (3+2) Annulations}

Enantioselective annulation reactions with an achiral cyclopropane system (acceptor-activated cyclopropane) were first described in 2005 by Sibi and co-workers (vide infra). Following this pioneering work, an important breakthrough was achieved by the group of Johnson with chiral D-A cyclopropanes. They reported in 2009 the enantioselective synthesis of tetrahydrofurans derivatives through a dynamic kinetic asymmetric $(3+2)$ annulation of racemic D-A cyclopropanes and aldehydes (Scheme 1). ${ }^{38}$ The development of this DYKAT (DYnamic Kinetic Asymmetric Transformation) strategy was later highlighted in a Perspective. ${ }^{39}$ Initial studies revealed that the $p$-methoxyphenyl (PMP) substituted diester cyclopropane was best suitable for a DYKAT as it undergoes fast ring opening under Lewis acid coordination. After examination of several pyBOX 
(bis(oxazolinyl)pyridyl) ligands with $\mathrm{MgI}_{2}$ as catalyst, 1 provided the tetrahydrofuran derivatives with the highest enantiopurity. Electron-rich, cinnamyl and aliphatic aldehydes undergo the annulation reaction with activated cyclopropanes such as $p$-methoxyphenyl and 2-thienyl substituted cyclopropanes furnishing cycloadducts such as 2-4 in good yields and enantioselectivities.

Scheme 1. Dynamic kinetic asymmetric $(3+2)$ annulation of racemic D-A cyclopropanes and aldehydes reported by Johnson and co-workers. ${ }^{38}$

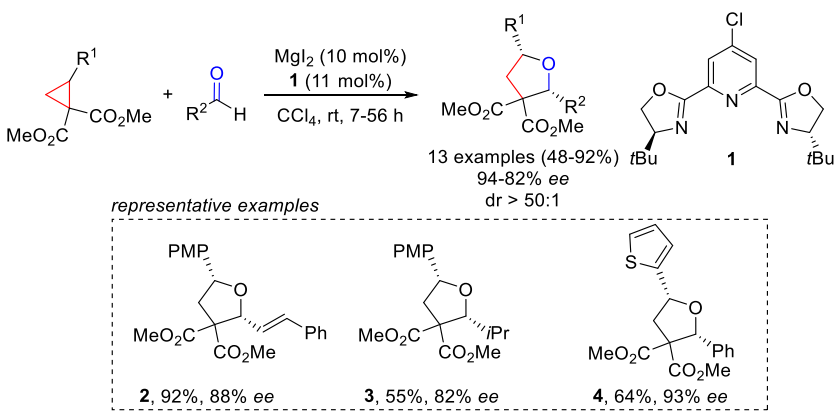

Racemization/epimerization of the starting material or a reaction intermediate is needed for a DYKAT process. The phenyl-substituted D-A cyclopropane $\left(\mathrm{R}^{1}=\mathrm{Ph}\right)$ is not reactive enough to allow racemization and is therefore suitable only for a kinetic resolution. Reaction on the racemic phenyl-substituted D-A cyclopropane revealed that the $(S)$-enantiomer reacts faster. Based on this observation and the stereochemistry of the product, the authors proposed a stereochemical model involving a magnesium-cyclopropane chelate complex displaying octahedral geometry (Figure 2). Even though steric interactions might occur between the $t \mathrm{Bu}$ and the aryl group in the complex formed by the $S$ enantiomer (complex I), the aldehyde's approach suffers from unfavorable steric interactions with the aryl group in the complex formed by the $R$ substrate (complex II), leading to slower reaction.
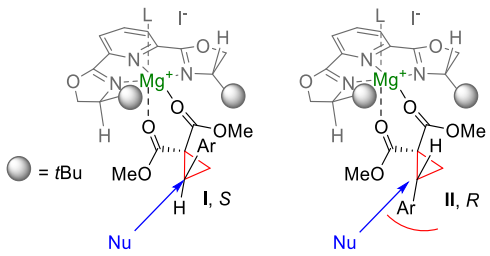

Figure 2. Proposed stereochemical model for the addition of aldehydes to activated D-A cyclopropanes.

The same group applied the DYKAT to the synthesis of 2,5-cissubstituted pyrrolidines from racemic cyclopropanes and $(E)$ aldimines (Scheme 2). ${ }^{40}$ Previous observations by Kerr and Tang showed that an $N$-benzyl protecting group for aldimines favors a cis selectivity. ${ }^{41,42}$ Using $\mathrm{MgI}_{2}$ with pyBOX 1 and alkoxy-substituted benzyl protecting groups, good yields and selectivities could be already obtained. By screening various 4$\mathrm{X}-t$ BupyBOX ligands, 4-Br- $t$ BupyBOX (5) was finally selected, as it furnished the pyrrolidine from challenging electron-rich aldimines with the highest yield and selectivity. Yields and enantioselectivities were high with both electronrich and -neutral aryl aldimines to give pyrrolidines such as 68. However, electron-poor aryl, alkenyl and aliphatic aldimines were not successful in this transformation.
Scheme 2. Asymmetric (3+2) annulation of D-A cyclopropanes with aldimines reported by Johnson and coworkers. $^{40}$

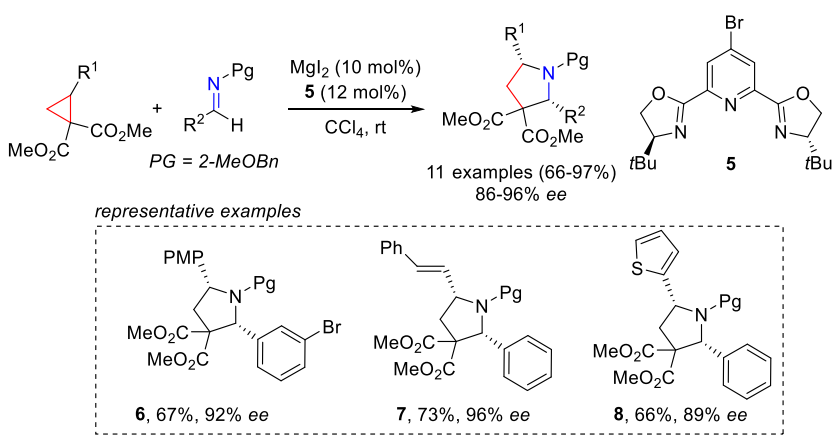

The breakthrough of Johnson's work served as inspiration for many other reports. The initially developed Mg-PyBox catalytic system was improved and other metals as well as other BOX ligands have been used. Tang and co-workers developed a copper-catalyzed enantioselective and diastereoselective annulation reaction of cyclic enol ethers with racemic D-A cyclopropanes allowing the synthesis of [n.3.0] carbocycles (Scheme 3). ${ }^{43}$ This reaction was exemplified with five-, six-, and seven-membered silyl enol ethers with paramethoxyphenyl- (PMP), 2-thiophenyl-, 3,4,5trimethoxyphenyl- (TMP), and alkenyl-substituted D-A cyclopropanes to give carbocycles such as 10-12. This work was also extended to benzene-fused silyl enol ethers furnishing cyclic products such as $\mathbf{1 3}$ with high yield and diastereoselectivities (>99:1). In addition to set the bicyclic skeleton, this method installs a tertiary alcohol at the ring junction and at least two stereocenters in one-step with good to excellent yield and high enantiomeric excess. Key for high enantiomeric excess was the introduction of a bulky aryl sidearm group (R) at the bridging carbon atom of the BOX (bis(oxazoline)) ligand (9) combined with adamantyl ester groups on the cyclopropanes. It is worth mentioning that a kinetic resolution could be applied to less reactive cyclopropanes (e.g. phenyl-substituted).

Scheme 3. Copper-catalyzed $(3+2)$ annulation of cyclic silyl enol ethers with D-A cyclopropanes reported by Tang and co-workers. ${ }^{43}$
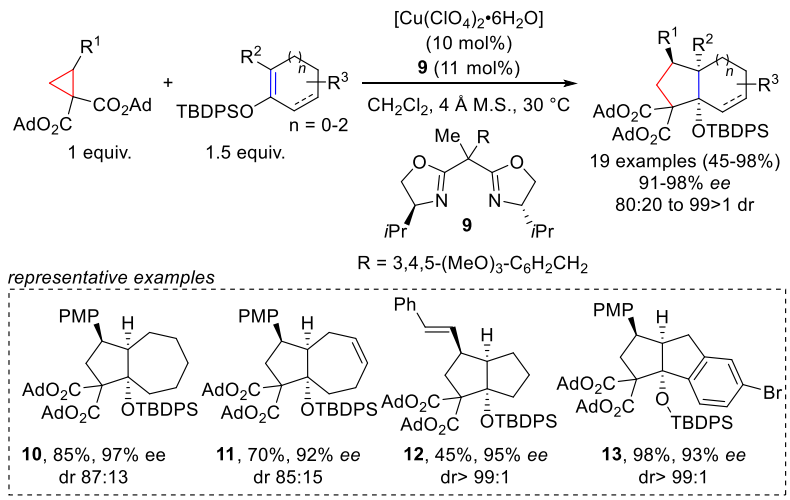

Our group described the first example of a copper-catalyzed $(3+2)$ annulation reaction of aminocyclopropane 14 with enol ethers and aldehydes through a DYKAT process (Scheme 4). ${ }^{44}$ The same copper catalyst bearing a $t \mathrm{BuBOX}$ ligand (15), is used 
both for the synthesis of cyclopentanes and tetrahydrofurans. The stereoselectivity and the efficiency of the reaction were improved by modifying the substituent on the nitrogen of the cyclopropane, by increasing the steric hindrance of the substituent on the ligand and finally by changing the counterion of the metal catalyst. Thus, the combination of succinimide, $t \mathrm{BuBOX}$ ligand and $\mathrm{Cu}\left(\mathrm{ClO}_{4}\right)_{2}$ as the copper source furnished cyclopentylamines, such as $\mathbf{1 6}$ or $\mathbf{1 7}$, and tetrahydrofuran derivatives, such as $\mathbf{1 8}$ or $\mathbf{1 9}$, with excellent yields and good enantio- and diastereoselectivities.

Scheme 4. Dynamic kinetic asymmetric $(3+2)$ annulation of aminocyclopropane 14 with enol ethers and aldehydes reported by Waser and co-workers. ${ }^{44}$

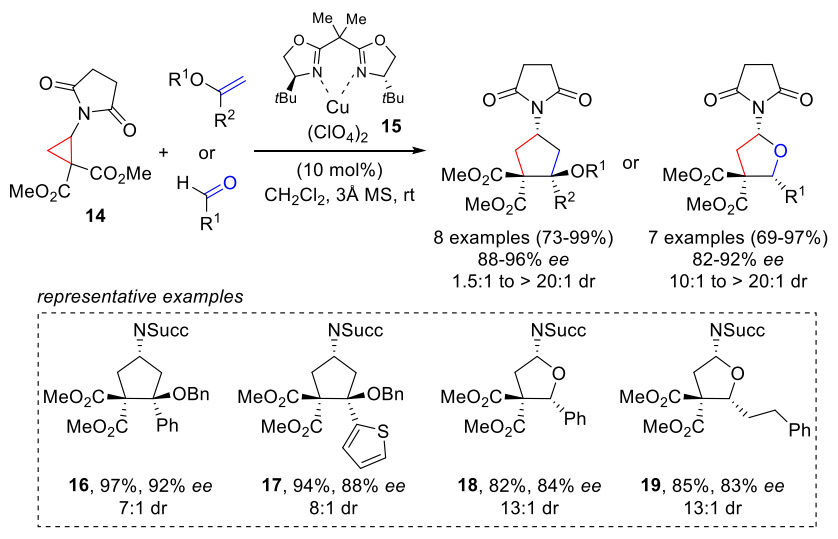

A speculative stereochemical model, disclosed in Figure 3, was also proposed: the nucleophile attack is faster in the complex formed by the $R$ enantiomer of cyclopropane 14 (complex I) whereas attack of the nucleophile is blocked by the tert-butyl substituents of the ligand in the complex formed by the $S$ enantiomer (complex II).
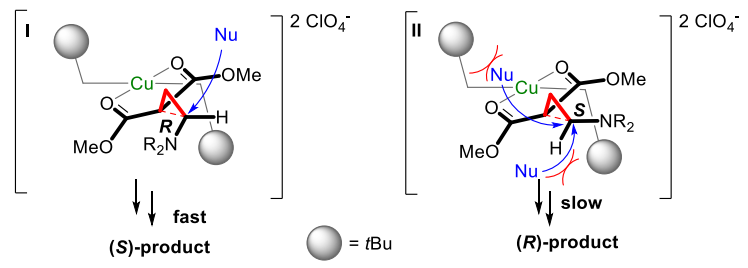

Figure 3. Proposed speculative stereochemical model for the $(3+2)$ annulation reaction of aminocyclopropanes. Reproduced with minor adaptation from Ref. 44, Copyright 2014, American Chemical Society.

As illustrated above classical $\pi$-systems (carbonyl, imines and olefins) were successfully used in enantioselective $(3+2)$ annulation reactions with D-A cyclopropanes. More challenging dearomatization reactions were also studied with indoles and benzazoles. The group of Tang described a coppercatalyzed asymmetric $(3+2)$ annulation of indoles with D-A cyclopropanes leading to enantioenriched C2,C3-fused indoline products such as 21-23 with excellent yields and diastereoselectivities (Scheme 5) ${ }^{45}$ Again, the modification of the side-arm group $\left(\mathrm{R}=\mathrm{Me}\right.$ and $\mathrm{R}^{\prime}=$ benzyl) allowed them to improve the catalyst activity as well as the enantioselectivity. The cagelike BOX ligand $\mathbf{2 0}$ with two tert-butyl groups at the meta position of the pendant benzyl groups was identified as the best ligand. The reaction tolerated substitutions on the indole motif and functionalized alkyl chains at the $\mathrm{C} 3$ positions $\left(\mathrm{R}^{1}\right)$ without major erosion of the enantioselectivity. The scope was extended to heteroaryl-, alkenyl-, and vinyl-substituted D-A cyclopropanes with good yields and enantioselectivities.

Scheme 5. Copper-catalyzed asymmetric $(3+2)$ annulation of indoles with D-A cyclopropanes reported by Tang and coworkers. $^{45}$

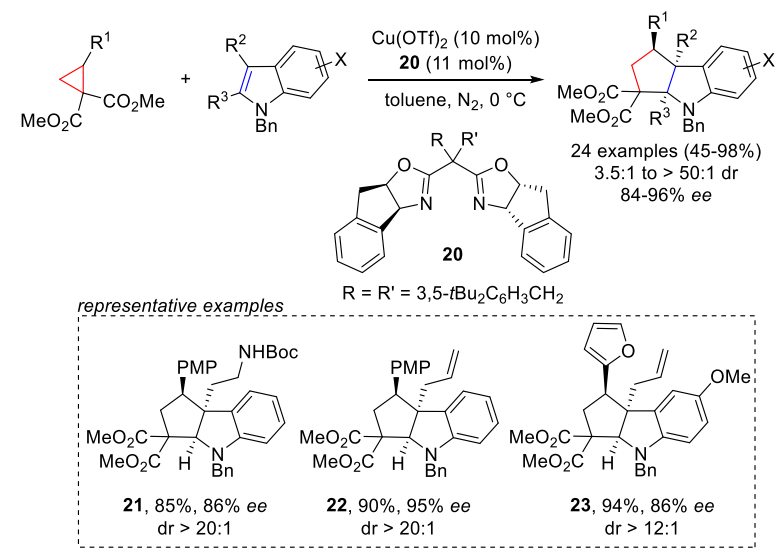

The authors also proposed a tentative stereochemical model based on the square-planar geometry of bisoxazoline copper complexes (Figure 4). The enantioinduction was explained by the favored approach of the $S i$ face of the indole to the $(R)$ cyclopropane in complex $\mathbf{I}$, thus avoiding steric interactions present in the complex II formed by the $S$ enantiomer.
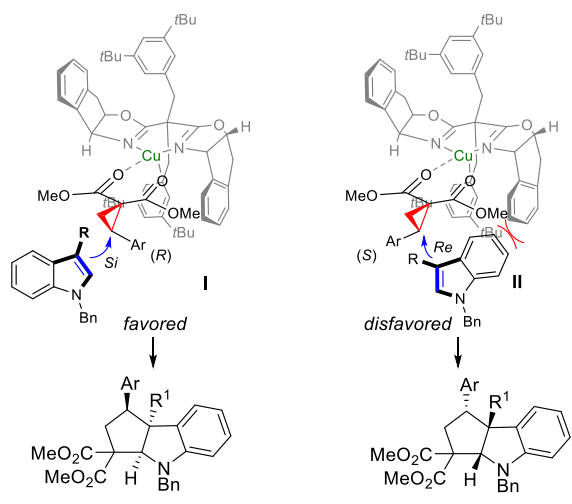

Figure 4. Proposed stereochemical model for the addition of indoles to activated D-A cyclopropanes.

Enantioselective dearomative $(3+2)$ annulations of benzothiazoles with D-A cyclopropanes were described by You and co-workers (Scheme 6). ${ }^{46}$ Here also, the DYKAT strategy was successfully applied leading to enantioenriched hydropyrrolo-thiazoles. The $t \mathrm{Bu}-\mathrm{PYBOX}$ ligand combined with $\mathrm{MgI}_{2}$ gave the best enantioselectivity when the reaction was performed at $0{ }^{\circ} \mathrm{C}$ in chlorobenzene. Overall, excellent yields and good $e e$ values were obtained for electron-rich or electron-deficient aryl cyclopropanes and benzothiazoles to give products such as 24-26. The authors also applied the same catalytic system in a kinetic resolution process. 
Scheme 6. Dearomative (3+2) annulations of D-A cyclopropanes with benzothiazoles reported by You and coworkers. $^{46}$
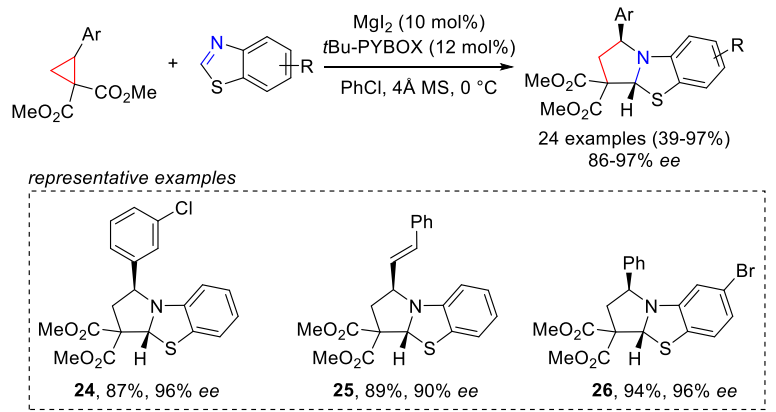

The same group published later a dearomative $(3+2)$ annulation reaction of benzazoles with aminocyclopropanes (Scheme 7A). ${ }^{47}$ Enantioenriched hydropyrrolo-benzazoles containing quaternary stereocenters were obtained via a kinetic resolution using $\mathrm{Cu}(\mathrm{OTf})_{2}$ as the copper source and the $t \mathrm{BuBOX}$ ligand. The use of succinimidyl cyclopropane 27 in excess (4 equiv.) was crucial to reach good yields and excellent enantioselectivities. Concerning the scope, benzothiazoles and benzoxazoles were suitable substrates giving dearomatized products such as 28-30 in high yields and enantioselectivities. Benzimidazoles showed some limitations, giving products such as $\mathbf{3 1}$ in only moderate yield and enantioselectivity. This catalytic asymmetric dearomatization (CADA) reaction was also applied by Guo and co-workers to purines generating enantioenriched dearomatized purine frameworks such as 3335 (Scheme 7B). ${ }^{48}$ This $(3+2)$ annulation was also found to be chemoselective when N9-alkenyl-substituted purines were used. The annulation products on the $\mathrm{C}=\mathrm{C}$ double bond at the N9-position were not observed.
Scheme 7. Enantioselective dearomative (3+2) annulations of benzazoles reported by You and co-workers (A) and of purines by Guo and co-workers (B) with aminocyclopropane $27 .{ }^{47,48}$

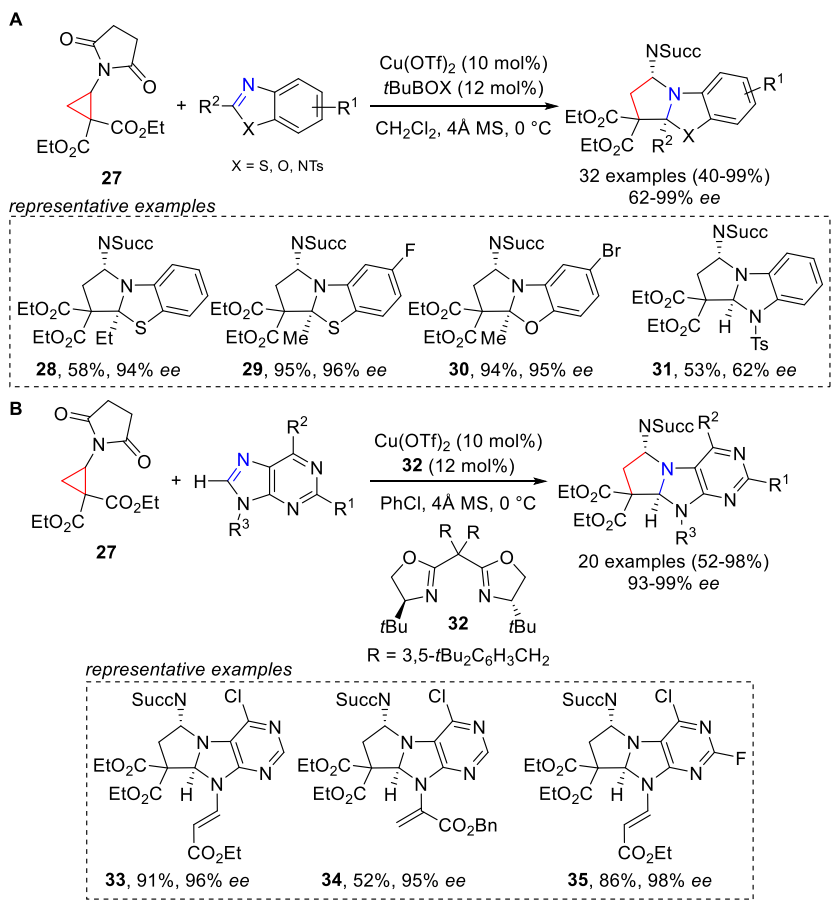

In addition, Baneerje and Verma attempted to apply their racemic $(3+2)$ annulation of D-A cyclopropanes with enamines to a dynamic kinetic asymmetric version. ${ }^{49}$ Unfortunately, low enantio- and diastereoselectivity were obtained.

All the examples above consist in the activation of the cyclopropane by a LUMO-lowering catalyst using a chiral metal complex. In $(3+2)$ annulation reactions, only two reports described the HOMO-raising activation of cyclopropanes through organocatalysis. First, a remarkable enantioselective organocatalytic $(3+2)$ annulation of racemic D-A cyclopropylketones with nitroolefins was described by Jørgensen and co-workers based on a new nucleophilic activation mode of D-A cyclopropanes combined with electrophilic activation of the nitroolefins using bifunctional urea-amine catalyst 36 (Scheme 8). ${ }^{50}$ Racemic di-cyano cyclopropylketones were activated by the basic pyrrolidine in 36, whereas the thiourea lowered the LUMO of the nitroolefin. From bifunctional derivatives obtained by varying the tertiary amine and the electronic properties of the aromatic ring on the urea, the para- $\mathrm{NO}_{2}$-substituted thiourea 36 delivered cycloadducts such as 37-40 in best yields and ee values, ranging from 66 to $91 \%$, which can be improved to $99 \%$ by recrystallization. The electron-withdrawing ability of the $\mathrm{NO}_{2}$ benzene substituent had a crucial role in increasing the hydrogen-donor character of the thiourea, leading to enhanced reactivity. 
Scheme 8. Brønsted base catalyzed (3+2) annulation of D-A cyclopropylketones with nitroolefins reported by Jørgensen and co-workers. ${ }^{50}$

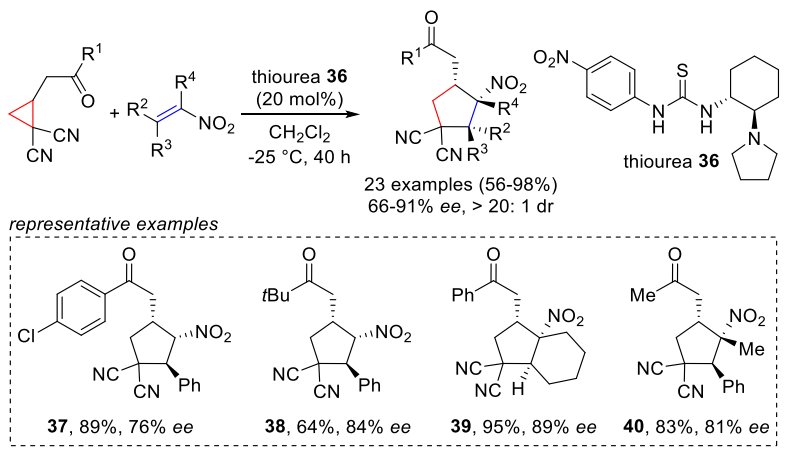

An enantioselective $(3+2)$ annulation between racemic D-A cyclopropanes and $\alpha, \beta$-unsaturated acyl fluoride catalyzed by a chiral NHC ( $N$-Heterocyclic Carbene) organocatalyst was described by Lupton and co-workers (Scheme 9). ${ }^{51}$ This reaction was proposed to occur via a $\alpha, \beta$-unsaturated acyl azolium intermediate generated through the reaction of the NHC catalyst with the acyl fluoride, at the same time releasing a fluoride anion, which activates the cyclopropane nucleophilically by desilylation. The chiral triazolylidene NHC catalyst 41 afforded the highest enantioselectivity. In terms of catalytic activity, the authors observed that electron-rich substituents on the nitrogen (e.g. alkyl or aryl) gave the expected product, while strongly electron-withdrawing substituents $\left(\right.$ e.g. $\left.\mathrm{C}_{6} \mathrm{~F}_{5}\right)$ resulted in an inactive catalyst. Catalyst 41 with a tert-butyl group on the nitrogen was therefore selected. Tricyclic products such as $\mathbf{4 2}$ and $\mathbf{4 3}$ were obtained with high enantioselectivities. Erosion of the $e e$ values was observed with $\beta$-alkyl $\alpha, \beta$-unsaturated acyl fluoride (product 44) and cinnamoyl fluorides bearing electron-poor substituents.

Scheme 9. NHC-catalyzed (3+2) annulation of racemic D-A cyclopropanes with $\alpha, \beta$-unsaturated acyl fluorides reported by Lupton and co-workers. ${ }^{51}$
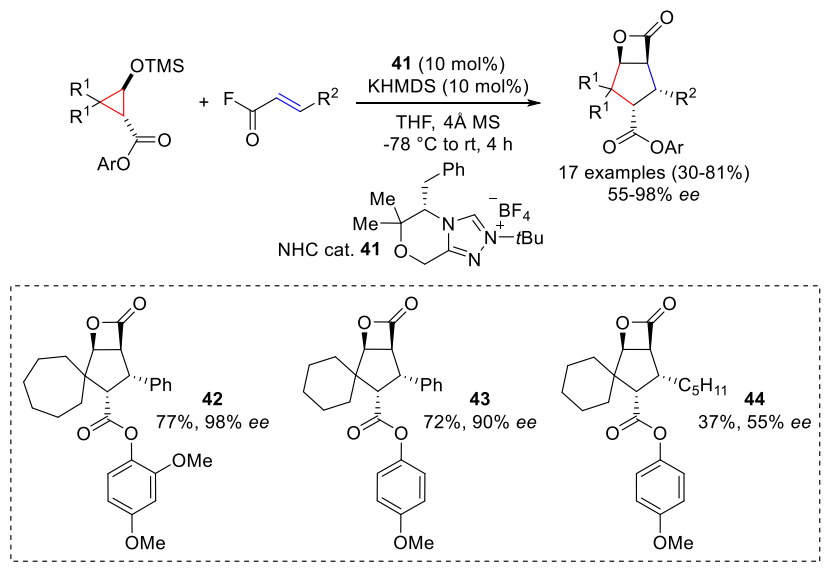

\subsection{2. $(3+3)$ Annulations}

Inspired by the work of Sibi on the enantioselective $(3+3)$ annulation of non-chiral cyclopropane diesters with nitrones (vide infra), Tang and co-workers published in 2007 an enantioselective and diastereoselective $(3+3)$ annulation of racemic D-A cyclopropanes with nitrones (Scheme 10). ${ }^{52}$ Application of their previously developed pseudo- $C_{3-}$ symmetric trisoxazoline (TOX) ligands ${ }^{53}$ with $\mathrm{Ni}\left(\mathrm{ClO}_{4}\right)_{2}$ provided tetrahydro-1,2-oxazine derivatives in good yields but low enantiomeric excess. Finally, trisoxazoline $\mathbf{4 5}$ possessing an improved sidearm group gave good yields, d.r. and $e e$ values. Methyl ester groups on the cyclopropane (product such as 46) gave lower enantioselectivities than benzyl ester groups (products such as 47). Vinyl- and styryl-substituted cyclopropanes could also be used in the reaction to give products such as $\mathbf{4 8}$ and 49 . This catalytic system could be applied to a kinetic resolution by reversing the cyclopropane/nitrone ratio. This provided an access to enantioenriched D-A cyclopropanes.

Scheme 10. Enantioselective $(3+3)$ annulation of D-A cyclopropanes with nitrones reported by Tang and coworkers. ${ }^{52}$

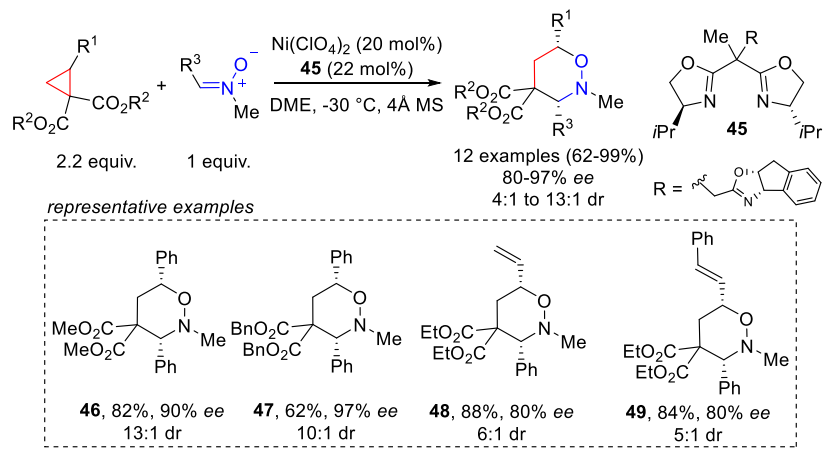

Zhou and co-workers reported the synthesis of optically active spirocyclic compounds (Scheme 11). ${ }^{54}$ Spirocyclopropyl oxindoles were opened through an enantioselective $(3+3)$ annulation with aldonitrones and ketonitrones with a BOX $(\mathbf{5 0}) / \mathrm{Ni}(\mathrm{OTf})_{2}$ chiral complex through a kinetic resolution. Amongst the screened protecting groups for the spirocyclopropyl oxindole, $N$-diethoxyphosphoryl led to the highest activity and diastereoselectivity. Spirocyclic products such as 51-54 were formed with high ee values, the starting cyclopropanes being recovered in good enantiopurity in most cases. Acetophenone-derived ketonitrones were also used giving spirocyclic oxindoles with an adjacent all-carbon quaternary stereocenter. A stepwise annulation mechanism was proposed to explain the observed diastereoselectivity, involving the $O$-attack of the nitrone to the cyclopropane coordinated by the chiral Lewis acid, followed by an intramolecular Mannich cyclisation. 
Scheme 11. Enantioselective $(3+3)$ annulation of spirocyclopropyl oxindoles with aldonitrones reported by Zhou and co-workers. ${ }^{54}$

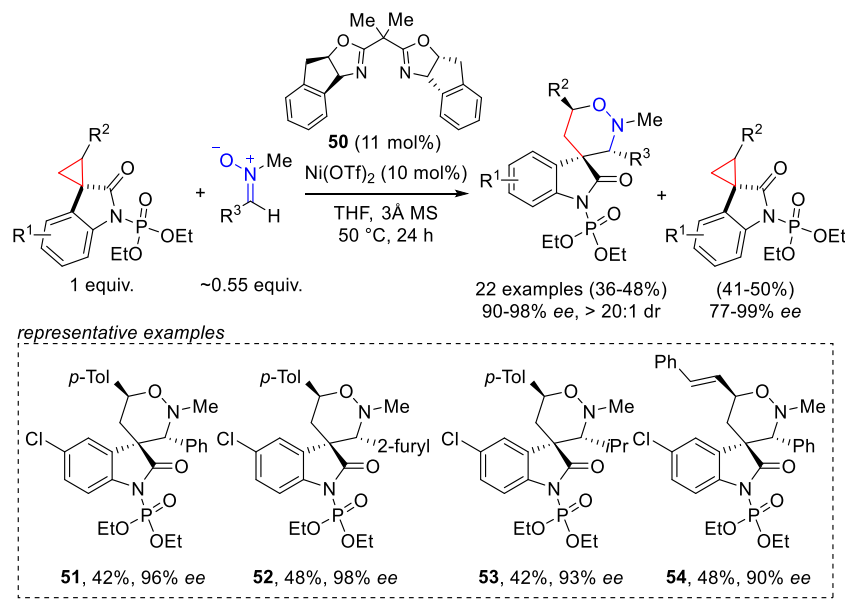

In their continuous search for enantioselective transformations based on the modification of BOX ligands using the sidearm strategy, the group of Tang also reported an enantioselective $(3+3)$ annulation of aromatic azomethine imines with D-A cyclopropanes (Scheme 12). ${ }^{55}$ After screening several ligands focusing on side-arm optimization and fine-tuning of the substrate, the TOX (trisoxazoline) ligand $\mathbf{5 5}$ combined with a sterically hindered neopentyl ester group on the cyclopropane gave the highest $e e$ values. Aryl, heterocyclic, styryl and vinyl donor groups $\left(\mathrm{R}^{1}\right)$ were well tolerated in this transformation leading to 6,6,6-tricyclic dihydroisoquinoline derivatives such as 56-59 in high yields and enantioselectivities and good diastereomeric ratios.

Scheme 12. Enantioselective $(3+3)$ annulation of aromatic azomethine imines with $D$-A cyclopropanes reported by Tang and co-workers. ${ }^{55}$

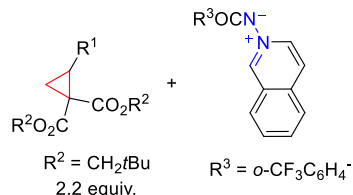

2.2 equiv.
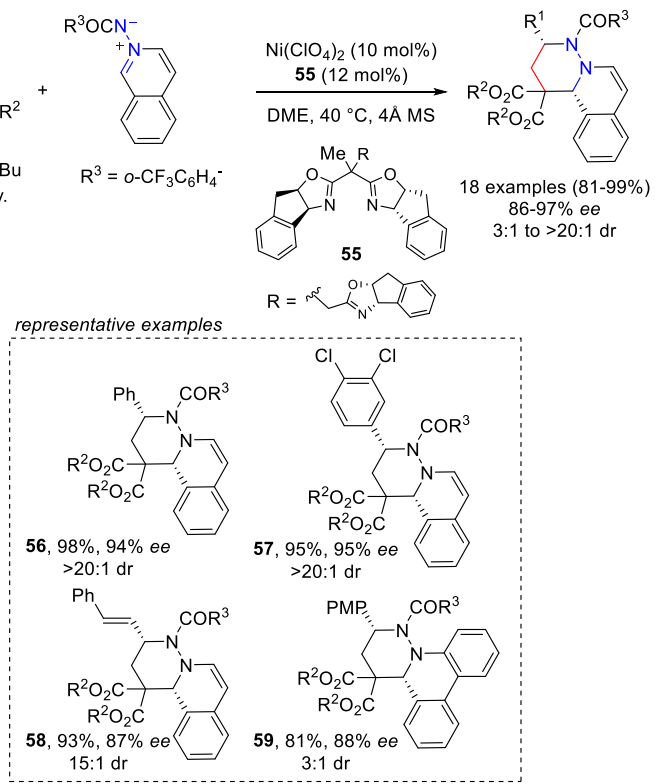

Based on DFT (Density-Functional Theory) calculations, a sixcoordinated $\mathrm{Ni}^{\mathrm{II}}$ complex was proposed to rationalize the observed stereoinduction (Figure 5). It involved the coordination of one molecule of azomethine imine acting as a ligand (L). The complex formed with the $S$ enantiomer was favored by $1.7 \mathrm{kcal} \mathrm{mol}^{-1}$ and displayed also a lower activation energy for attack by the nucleophile. In the transition state, the aryl group of the cyclopropane is placed above the $\mathrm{Ni}-N, N$ plan to avoid steric interactions with a second ligated isoquinoline azomethine imine (L). From the calculations, a $\pi-\pi$ stacking interaction was also revealed between the indane group of the sidearm and the phenyl group of the cyclopropane.

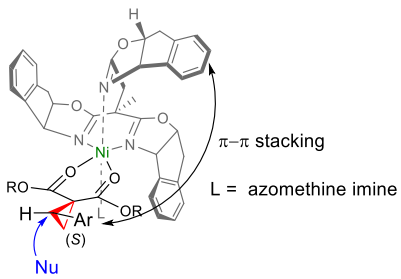

Figure 5. Proposed stereoinduction model for the attack of azomethine imines onto activated D-A cyclopropanes.

The same group described the one-pot enantioselective $(3+3)$ annulation of 2-alkynylindoles and D-A cyclopropanes furnishing optically active tetrahydrocarbazoles (Scheme 13). ${ }^{56}$ They started their optimization with the asymmetric ringopening reaction. BOX ligand $\mathbf{6 0}$ with copper(II) triflate was found to give good enantioinduction for product such as $\mathbf{6 1}$. Next, the one-pot $(3+3)$ annulation reaction was accomplished by treating the ring-opening product with indium(III) chloride and DBU (1,8-Diazabicyclo[5.4.0]undec-7-ene) as additive. Tetrahydrocarbazoles such as $\mathbf{6 2}$ and $\mathbf{6 3}$ were obtained in good yields and moderate to good enantioselectivities. Similar to the asymmetric induction model disclosed in Figure 4, the authors suggested that the side arm groups block the upper and lower sides of the $\mathrm{Cu}$ center and that the cyclohexyl groups hinder the top-left and low-right corners. Therefore, the discrimination of $S$ and $R$ cyclopropanes would arise from steric repulsions between the sterically hindered indole and the cyclohexyl substituent of the ligand (see complex I in Figure 4).

Scheme 13. One-pot stepwise enantioselective $(3+3)$ annulation of 2-alkynylindoles and D-A cyclopropanes reported by Tang and co-workers. ${ }^{56}$

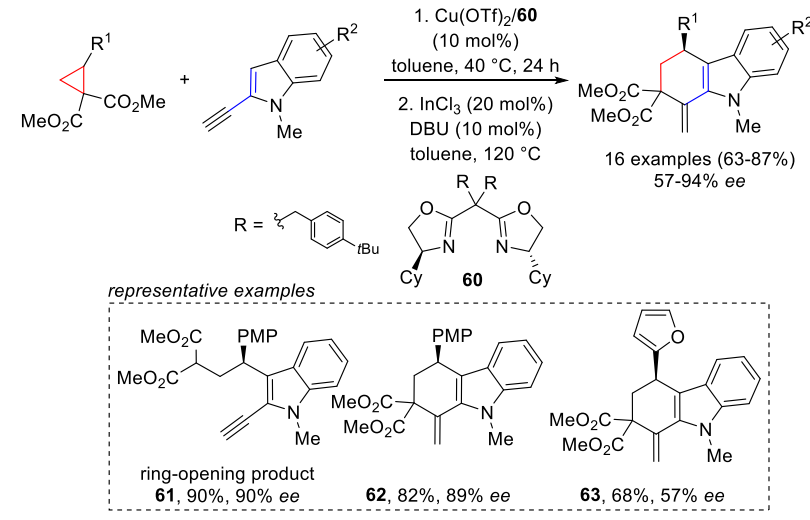

Feng and co-workers developed an enantioselective $(3+3)$ annulation reaction of D-A cyclopropanes with mercaptoacetaldehyde in the presence of $N, N^{\prime}$-dioxide ligand 64 and $\mathrm{Sc}(\mathrm{OTf})_{3}$ (Scheme 14). ${ }^{57}$ Tetrahydrothiopyranols such as 65-67 were formed with moderate yields and excellent 
enantiomeric excesses starting from aryl-substituted D-A cyclopropanes. However, vinyl-substituted cyclopropanes were not successful in this reaction. The enantioselectivity was drastically improved by using sterically hindered aryl substituents on the amide groups of the $N, N^{\prime}$-dioxide.

Scheme 14. Enantioselective $(3+3)$ annulation of D-A cyclopropanes with mercaptoacetaldehyde reported by Feng and co-workers. ${ }^{57}$

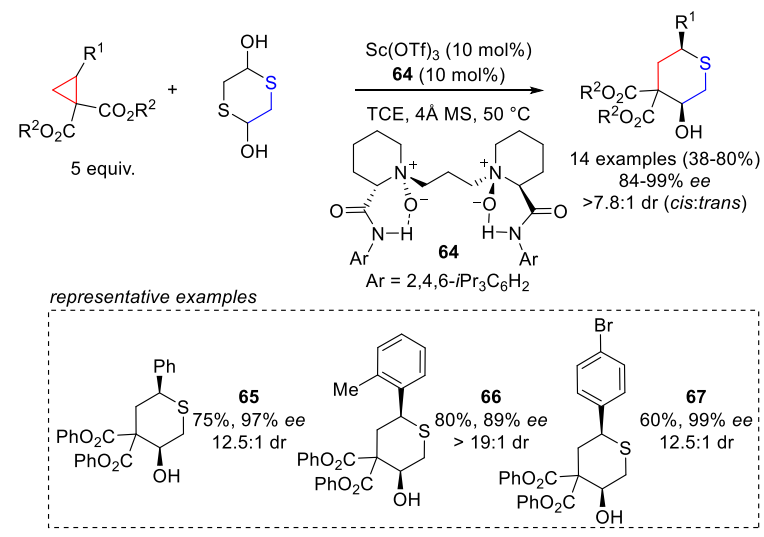

\subsubsection{Other annulations}

The group of Tang described an enantioselective coppercatalysed (4+3) annulation of D-A cyclopropanes with dienolsilyl ethers using a chiral trisoxazoline ligand (Scheme 15). ${ }^{58}$ This transformation is particularly challenging as the $(3+2)$ annulation product is usually obtained. Cycloheptenes and $[\mathrm{n}, 5,0]$ carbobicycles such as 69-72 were produced selectively in good yields and good enantioselectivies in presence of TOX ligand $\mathbf{6 8}$. NMR studies suggested a stepwise mechanism starting by a kinetically driven $(3+2)$ annulation, which is followed by a ring opening of the cyclopentane product and an intramolecular cyclization to afford the more thermodynamically stable $(4+3)$ cycloadduct.

Scheme 15. Enantioselective copper-catalysed (4+3) annulation of D-A cyclopropanes with dienolsilyl ethers reported by Tang and co-workers. ${ }^{58}$

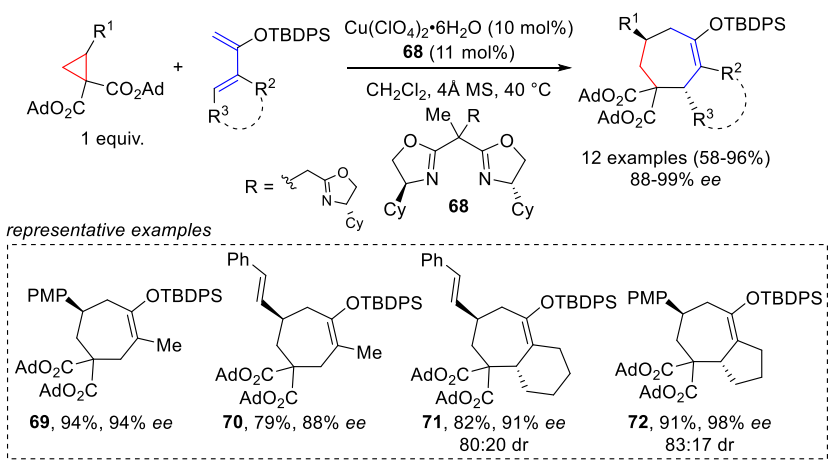

Vicario and co-workers described an enantioselective (4+2) annulation of alkylideneoxindoles with formyl cyclopropanes upon activation with a chiral $N$-heterocyclic carbene catalyst via a less common Umpolung approach (Scheme 16). ${ }^{59}$ The addition of NHC catalyst $\mathbf{7 3}$ on the aldehyde group of the cyclopropane followed by formation of the Breslow intermediate leads to a ring-opening event resulting in a bisnucleophilic synthon. The Breslow intermediate then reacts in a $(4+2)$ annulation with the alkylideneoxindole to give tetrahydropyrano[2,3- $b$ ]indoles such as 74-76 in excellent enantiomeric excesses and good yields. $\beta, \gamma$-Unsaturated- $\alpha$-keto esters were also applied successfully as Michael acceptors in this annulation.

Scheme 16. NHC-catalysed (4+2) annulation of alkylideneoxindoles with formyl cyclopropanes reported by Vicario and co-workers. ${ }^{59}$

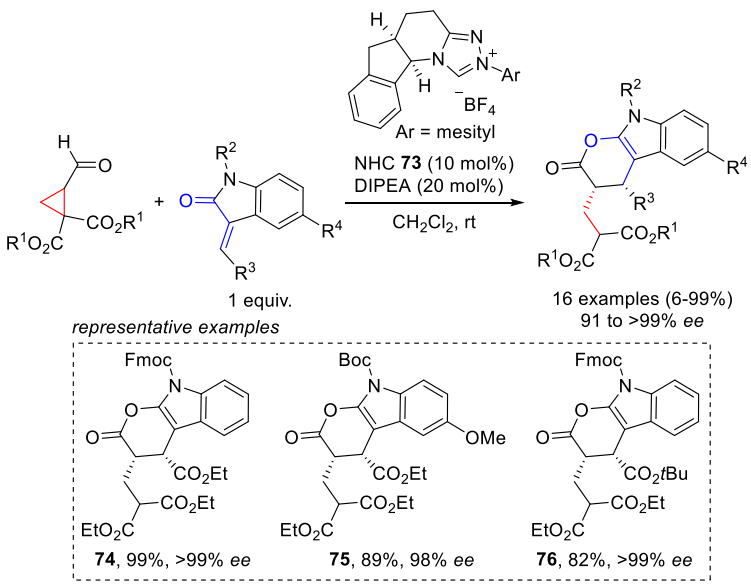

Jørgensen and co-workers reported an unexpected $(2+2)$ annulation based on enamine activation of cyclopropylacetaldehydes (Scheme 17) ${ }^{60}$ The authors observed that the in situ formed enamine-diester D-A cyclopropane is functionalized at the usually inert unsubstituted position to form spirocyclobutaneoxindoles such as $\mathbf{7 8 - 8 0}$ and spirocyclobutanebenzofuranones such as $\mathbf{8 1}$ in good yields and excellent enantioselectivities in the presence of chiral aminocatalyst $\mathbf{7 7}$. For practical reasons, a Wittig reaction was performed after the organocatalytic reaction. This unexpected transformation was rationalized through a stepwise mechanism leading to the formal $(2+2)$ annulation product, starting with the formation of dienamine $\mathbf{I}$, followed by a $(2+2)$ annulation with the alkene of the oxindole. 
Scheme 17. (2+2) Annulation of cyclopropylacetaldehydes with alkenyl oxindoles and benzofuranones reported by Jørgensen and co-workers. ${ }^{60}$

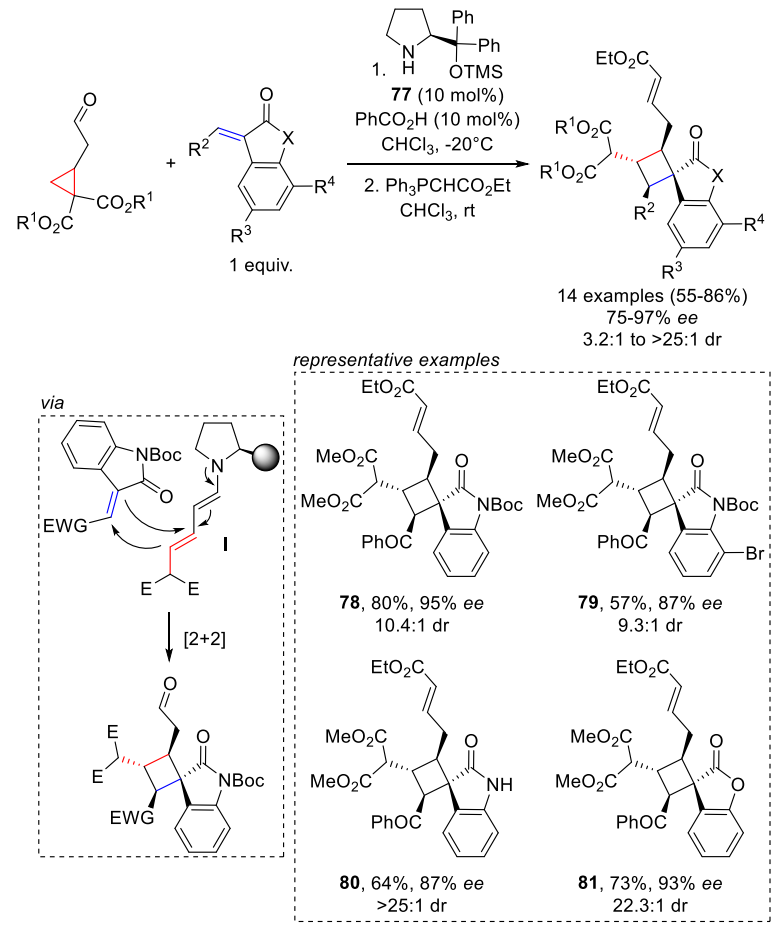

\subsection{Ring-opening reactions}

Enantioselective ring-opening reactions of D-A cyclopropanes have been studied using heteroatom nucleophiles. Secondary and even primary amines have been successfully applied in the synthesis of enantioenriched $\gamma$-aminobutyric acid (GABA) derivatives. Other nucleophiles include alcohols, water and thiols. D-A cyclopropanes have been also used in Friedel-Crafts alkylations of indoles and naphthols. The last example of ringopening reactions is an enantioselective desymmetrizing fragmentation of meso-cyclopropanes.

\subsubsection{Addition of heteroatom nucleophiles}

Based on the sidearm strategy, Tang and co-workers developed an enantioselective ring-opening reaction of D-A cyclopropanes with aliphatic amines leading to $\gamma$-substituted $\gamma$ aminobutyric acid (GABA) derivatives (Scheme 18). ${ }^{61}$ To overcome the complexation of the amine with the Lewis acid, a pendant coordinating group was installed on the BOX ligand. It was found that the indane trisoxazoline (In-TOX, 55) improved both the reaction rate and the enantiocontrol. Several GABA derivatives such as $\mathbf{8 2 - 8 5}$ were produced in excellent yields and enantioselectivities from aliphatic amines and aryl-, thienyl-, alkenyl- and vinyl- cyclopropanes. The catalytic system was also found to be efficient for a kinetic resolution process.
Scheme 18. Ni-catalyzed asymmetric ring opening reaction of D-A cyclopropanes with aliphatic amines reported by Tang and co-workers. ${ }^{61}$

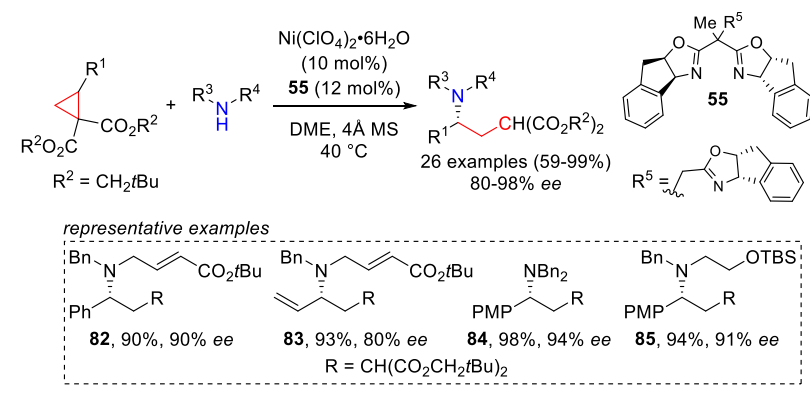

A model was proposed for asymmetric induction and was in agreement with an X-ray structure of a similar Ni complex. In this model (Figure 6), the $S$ enantiomer of the racemic cyclopropane binds selectively to the catalyst avoiding steric repulsions between the phenyl group and the indanyl moiety (Complex I). According to the authors, the sidearm group could enhance this preference. In order to investigate the role of the chiral sidearm, the reaction was further studied with other types of trisoxazoline and bisthiazoline ligands in which the chirality was installed on the sidearm and not on the parent framework. However, although these new ligands promoted the ringopening reaction, the GABA derivatives were obtained with only modest enantioselectivities. ${ }^{62}$
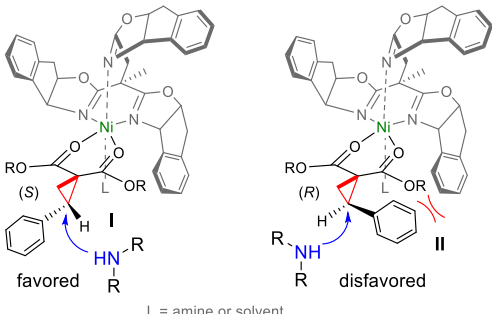

Figure 6. Proposed model for stereochemical induction for the attack of amines on activated D-A cyclopropanes.

In the study of Tang and co-workers, only secondary amines were used. The use of primary amines in ring-opening reactions of D-A cyclopropanes is challenging, as the obtained secondary amines can react further. Recently, Wang and co-workers described a ring-opening reaction with primary arylamines by using a chiral heterobimetallic catalyst (Scheme 19). ${ }^{63}$ Enantioenriched $\gamma$-amino acid derivatives such as 87-90 were produced in good yields by combining $\mathrm{Y}(\mathrm{OTf})_{3}$ and the metal binaphthyl phosphate catalyst $(R)-\mathrm{Yb}[\mathrm{P}]_{3}(\mathbf{8 6})$. Only traces of the ring-opened products were obtained in the presence of $\mathrm{Y}(\mathrm{OTf})_{3}$ or $(R)-\mathrm{Yb}[\mathrm{P}]_{3}$ alone, showing the unique activity of this bimetallic system. X-ray diffraction studies on the $\mathrm{Yb}(\mathrm{OTf})_{3}-\mathrm{Yb}[\mathrm{P}]_{3}$ adduct revealed a symmetrical binuclear metal complex in which two metals share four bridged phosphate ligands. Finally, experimental and computational studies suggested that this ring-opening reaction is a kinetic resolution involving an $\mathrm{S}_{\mathrm{N}} 2$-like mechanism. The calculations indicated that the binding of the $R$ cyclopropane to the $\mathrm{Yb}$ site is favored by 3-6 $\mathrm{kcal} \cdot \mathrm{mol}^{-1}$ over the $\mathrm{S}$ enantiomer, leading to ring-opening of the former. 
Scheme 19. Asymmetric ring-opening reaction of D-A cyclopropanes with primary amines catalyzed by a chiral heterobimetallic complex reported by Wang and coworkers. ${ }^{63}$

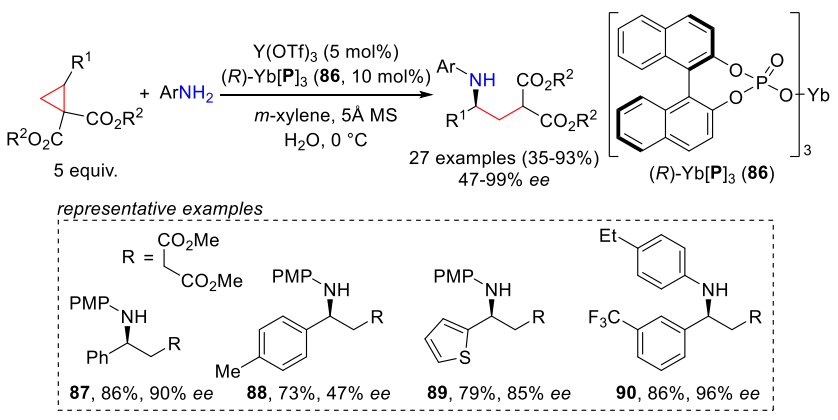

The group of Tang also extended their method to the synthesis of optically active $\gamma$-substituted $\gamma$-hydroxybutyric acid (GHB) derivatives with benzyl alcohols as nucleophiles by employing the Cy-TOX (68)/Cu(II) catalyst system (Scheme 20). ${ }^{64}$ This ring-opening reaction led to GHB products with good yields and high enantioselectivities.

Scheme 20. Cu-catalysed asymmetric ring-opening reaction of D-A cyclopropanes with alcohols reported by Tang and co-workers. ${ }^{64}$

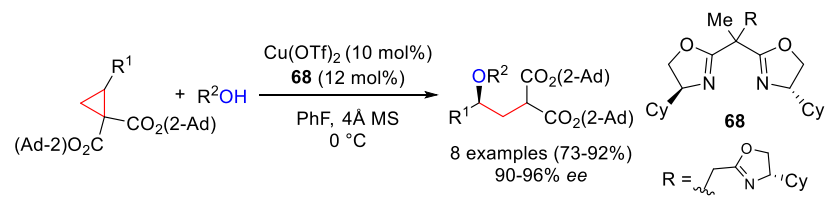

They then developed a direct approach for the synthesis of GHB derivatives using water as nucleophile (Scheme 21). The development of an asymmetric ring-opening reaction of D-A cyclopropanes with water is particularly challenging as it can poison the catalyst and the formed alcohol product might cause competing ring-opening reactions. Key to the success was the use of a copper hydrate source which acts both as a Lewis acid and a reservoir of water for the ring-opening reaction. The reaction was also run with $\mathrm{Cu}\left(\mathrm{ClO}_{4}\right)_{2} \bullet 6 \mathrm{H}_{2}{ }^{18} \mathrm{O}$ furnishing isotopic labelled GHB derivatives such as 91-93 with high enantiopurities.

Scheme 21. Enantioselective ring-opening reaction of D-A cyclopropanes with water reported by Tang and coworkers. $^{64}$

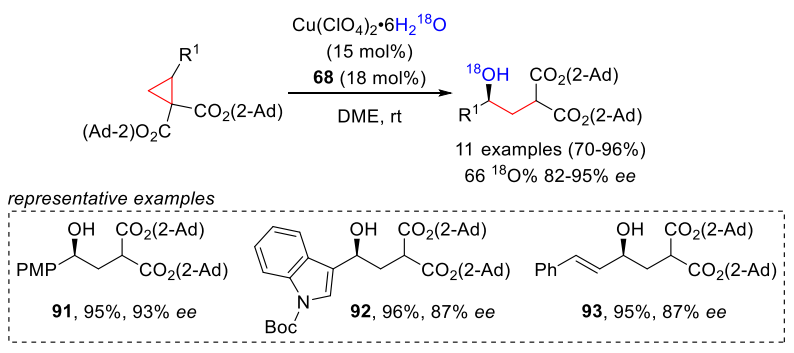

Feng and co-workers developed an asymmetric ring-opening reaction with several nucleophiles using a chiral $N, N$ '-dioxide (64)/scandium (III) catalyst (Scheme 22) ${ }^{65}$ A series of thiols, carboxylic acids and alcohols gave opened products such as 94 97 with good yields and generally high enantiomeric excess with a single catalytic system. The enantioselectivity was moderate for carboxylic acids (product 96) and dropped considerably with phenols (product 97).

Scheme 22. Asymmetric ring-opening of D-A cyclopropanes with thiols, alcohols and carboxylic acids reported by Feng and co-workers. ${ }^{65}$

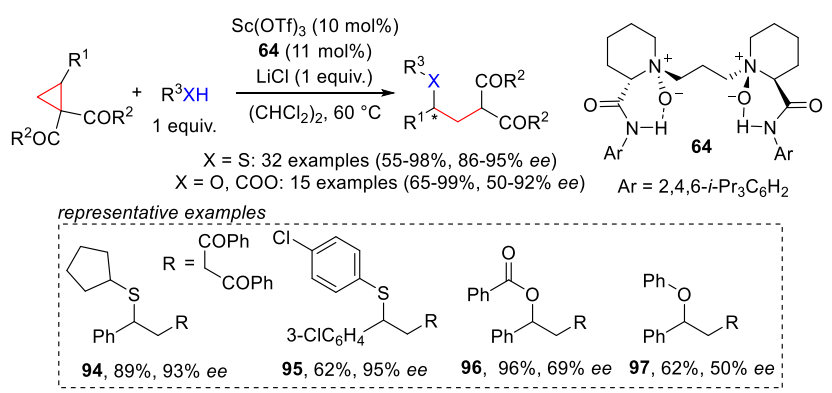

\subsubsection{Friedel-Crafts alkylations}

Johnson and co-workers developed in 2013 an asymmetric Friedel-Crafts alkylation reaction of indoles with D-A cyclopropanes via a DYKAT process (Scheme 23). ${ }^{66}$ The more sterically encumbered TBS-protected indoles furnished the homo-Michael adduct with the best yields and enantiomeric ratios in the presence of pyBOX ligand 5. A series of indoles with diverse substituents as well as thienyl-, furanyl- and styrenyl-substituted D-A cyclopropanes were tolerated providing enantioenriched alkylated products such as 98-100 in good yields. However, D-A cyclopropanes bearing a 2-OMePh or a phthalimide group were not successful. In contrast, the less reactive phenyl-substituted cyclopropane was suitable for a kinetic resolution.

Scheme 23. Enantioselective Friedel-Crafts alkylation of indoles with D-A cyclopropanes reported by Johnson and co-workers. ${ }^{66}$

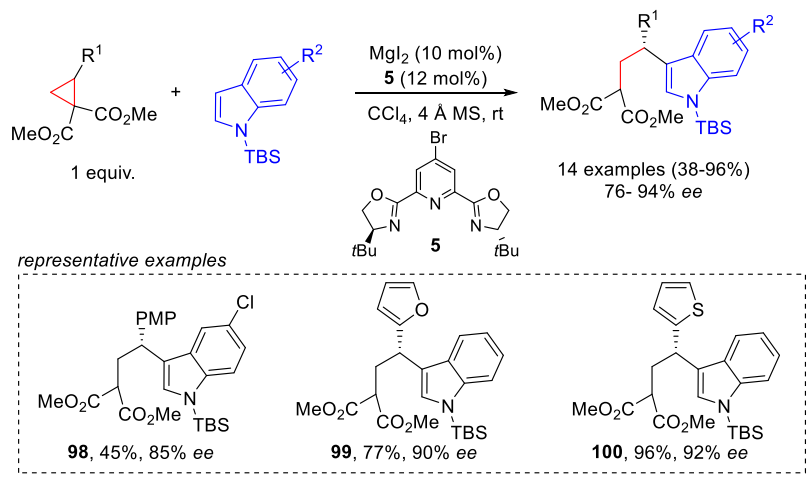

The group of Feng applied their catalytic scandium system to the same Friedel-Crafts reaction via a kinetic resolution (Scheme 24). ${ }^{67}$ The chiral $N, N$ '-dioxide (64)/scandium (III) catalyst in the presence of $\mathrm{MgCl}_{2}$ could be used for various substituted $N$-methylindoles to give alkylated products such as 101-104 in moderate to excellent yields and good enantiomeric excesses. The influence of $\mathrm{MgCl}_{2}$ in the reactivity, as well as the stereoselectivity, were rationalized by a counterion effect. 
Scheme 24. Enantioselective Friedel-Crafts alkylation of indoles reported by Feng and co-workers. ${ }^{67}$

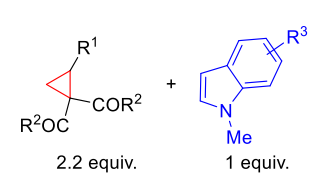

$$
\begin{gathered}
\mathrm{Sc}(\mathrm{OTf})_{3}(10 \mathrm{~mol} \%) \\
\mathbf{6 4}(10 \mathrm{~mol} \%) \\
\mathrm{MgCl}_{2}(1 \text { equiv. })
\end{gathered} \underset{\mathrm{CHCl}_{3}, 35^{\circ} \mathrm{C}}{\longrightarrow}
$$

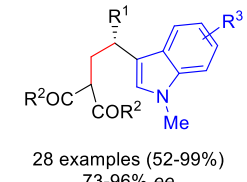
$73-96 \%$ ee representative examples

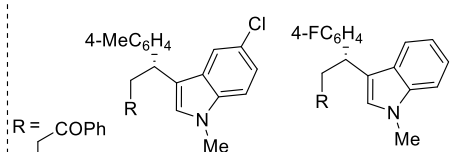

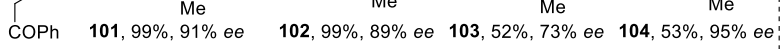

The chiral $N, N$ '-dioxide (64)/scandium (III) complex was also applied to the alkylation of 2-naphthols by the same group. ${ }^{68}$ Here, the conditions required higher temperatures and longer reaction times due to the lower nucleophilicity of 2-naphthols.

A copper-catalyzed enantioselective Friedel-Crafts alkylation of indoles was described by our group via a desymmetrization of meso-diaminocyclopropane $\mathbf{1 0 5}$ (Scheme 25). ${ }^{69}$ The development of novel $N$-substituted meso-cyclopropane $\mathbf{1 0 5}$ as well as the new BOX ligand 106, bearing bulky diarylmethanol groups, was essential to achieve high enantioselectivities. The free hydroxy group of $\mathbf{1 0 6}$ led to enhanced reactivity, allowing running the reaction at low temperature $\left(-50{ }^{\circ} \mathrm{C}\right)$. Enantioenriched urea products such as 107-111 were thus obtained in good yields and excellent diastereoselectivities. Indoles bearing electron-withdrawing or electron-donating substituents and a TIPS-protected pyrrole (product 111) were successful in this transformation.

Scheme 25. Friedel-Crafts alkylation of indoles through desymmetrisation of aminocyclopropanes reported by Waser and co-workers. ${ }^{69}$

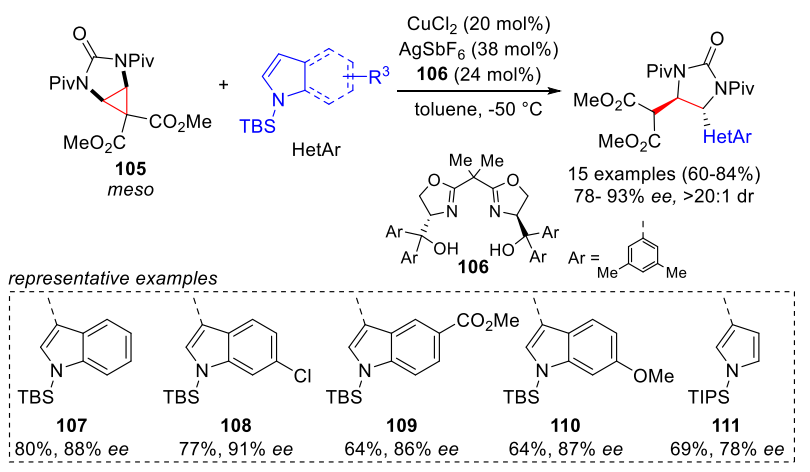

Based on the absolute configuration of the Friedel-Crafts products and the activating effect of the hydroxy group, a highly speculative stereochemical model was proposed (Figure 7). In this model, the tert-butyl of the pivaloyl moieties orientates in an opposite direction to the aryl groups of the ligand and may act as a relay for stereoinduction. One of the electrophilic carbon atoms of the cyclopropane is then blocked with a tertbutyl group.

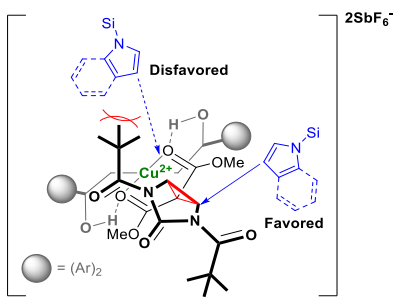

Figure 7. Proposed stereochemical model for the addition of indoles to activated meso-cyclopropane 105 . Reproduced with minor adaptation from Ref. 69. Copyright 2018, John Wiley and Sons.

D-A aminocyclopropanes have also been used by Guo and coworkers in the copper-catalyzed asymmetric Friedel-Crafts alkylation of $\beta$-naphthols using bisoxazoline ligand 112 (Scheme 26). ${ }^{70}$ In contrast to the alkylation of naphthols with aryl-substituted cyclopropanes reported by Feng and coworkers, ${ }^{68}$ both $C$ - and $O$-alkylations were observed with aminocyclopropanes with a chemoselectivity in favor of the $C$ alkylation. A range of $\gamma$-aryl GABA derivatives such as 113116 was produced in good yields and high $e e$. The minor $O$ alkylation products were also produced in high enantiomeric excess. Additionally, 1-methylindole reacted also to give the alkylation product in good yield and enantioselectivity.

Scheme 26. Friedel-Crafts alkylation of $\beta$-naphthols with aminocyclopropanes reported by Guo and co-workers. ${ }^{70}$

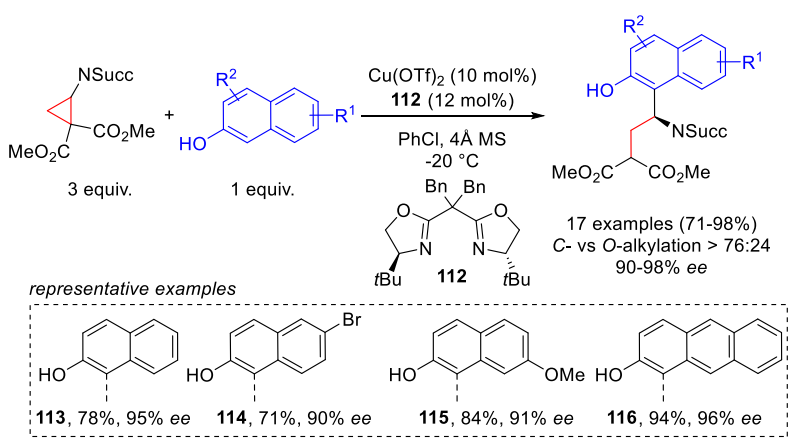

\subsubsection{Base-mediated fragmentation}

Jørgensen and co-workers published in 2009 an enantioselective organocatalytic desymmetrization of mesocyclopropane 117 through a chiral base-mediated fragmentation (Scheme 27)..$^{71}$ The bifunctional quinidine-derived thiourea catalyst 118 induces a selective E1cb elimination to give the optically active elimination product 119. The proposed mechanism, illustrated in Scheme 27 (transition state I), involves hydrogen bonding between the carbonyl and the thiourea, which increases the acidity of the $\alpha$-hydrogen atom and promotes the stereoselective deprotonation by the quinuclidine base. 
Scheme 27. Enantioselective desymmetrization of mesocyclopropane 117 through a chiral base-mediated fragmentation reported by Jørgensen and co-workers. ${ }^{71}$

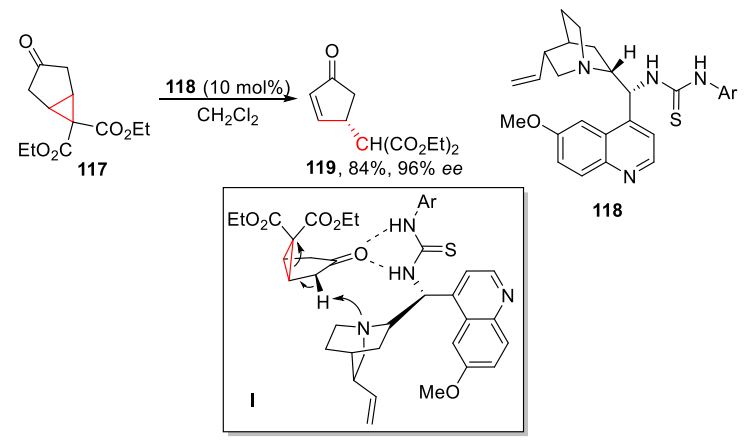

2.3. Other ring-opening/closing processes

More complex ring-opening/closing processes of D-A cyclopropanes not involving simple annulation with $\pi$ partners have mainly involved the use of primary amines and bisnucleophiles. These types of transformations allow multiple bond formation and therefore a fast access into molecular complexity.

\subsubsection{Cloke-Wilson rearrangement}

Amongst the rearrangement of cyclopropanes, the ClokeWilson rearrangement converts a strained cyclopropyl ketone to a more stable dihydrofuran under thermal conditions. ${ }^{72,73}$ In other words, this process occurs without the addition of an external nucleophile and involves ring-opening/closing events. Recently, Vicario and co-workers described the first catalytic enantioselective Cloke-Wilson rearrangement of racemic D-A cyclopropanes bearing ester-substituted cyclopropyl ketones based on the use of chiral phosphoric acid 120 (Scheme 28A). ${ }^{74}$ The latter protonates the ketone leading to a carbocationic intermediate that undergoes cyclization. Hydrogen bonding and ion-pairing interactions between the phosphate and this intermediate are believed to be responsible for stereoinduction. Computational and experimental studies supported this hypothesis and confirmed that this rearrangement occurred via a DYKAT process. Dihydrofurans such as 121-124 were obtained with excellent yields and good enantiomeric ratios. The scope was then extended to cyclopropyl ketones with only one electron-withdrawing group using chiral phosphoric acid 125 (Scheme 28B, products 126-128).
Scheme 28. Catalytic enantioselective Cloke-Wilson rearrangement of racemic $D$-A cyclopropanes reported by Vicario and co-workers. ${ }^{74}$

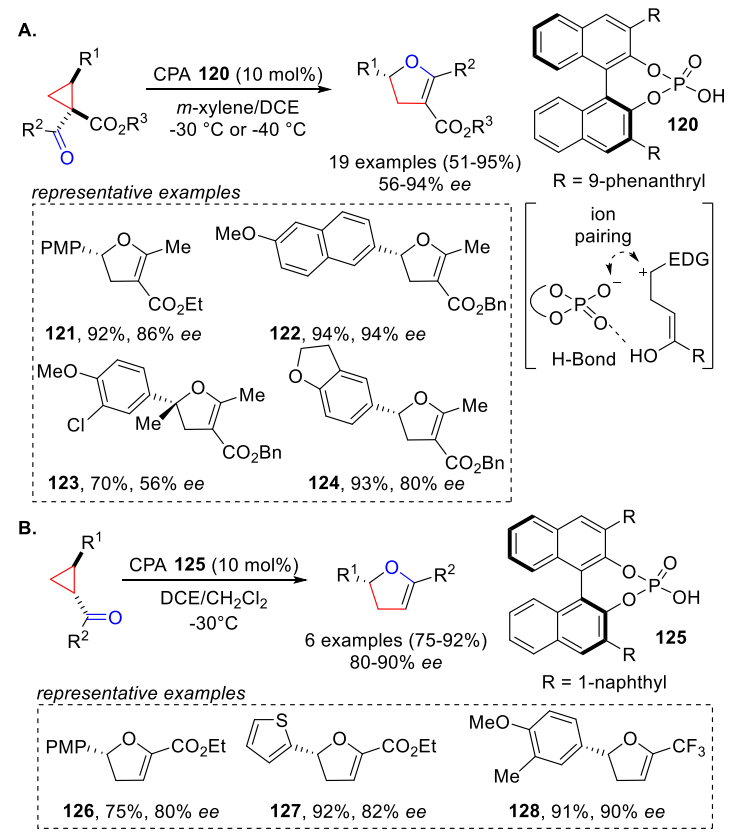

\subsubsection{Ring opening/cyclization with primary amines}

Feng and co-workers described the enantioselective synthesis of dihydropyrroles through a ring opening/cyclization sequence using primary amines (Scheme 29). ${ }^{75}$ The $N, N^{\prime}$-dioxide (64)/Sc(III) catalyst system provided an access to enantioenriched 2,4,5-trisubstituted 2,3-dihydropyrroles such as 129-133 with good yields. The scope is broad, including various aryl- and vinyl-substituted cyclopropanes as well as substituted aryl amines. Aliphatic amines were not successful except for cyclopropyl amine.

Scheme 29. Asymmetric ring-opening/cyclization of D-A cyclopropanes with primary amines reported by Feng and co-workers. ${ }^{75}$

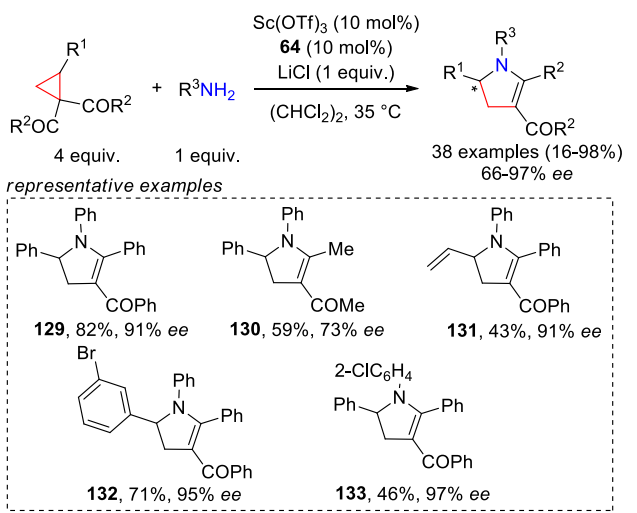

An asymmetric ring-opening/cyclization/retro-Mannich reaction of D-A cyclopropanes with aryl 1,2-diamines was also developed by the same group (Scheme 30$).{ }^{76}$ Once the hemiaminal $\mathbf{I}$ is produced, the second primary amine reacts through a 5-exo-tet cyclisation to give aminal $\mathbf{I I}$, which is followed by a retro-Mannich reaction delivering benzimidazole derivatives 
such as 134-137. Here, scandium(III) chloride hydrate combined with $N, N$ '-dioxide ligand 64 gave the best yields and enantioselectivities. $\mathrm{Sc}(\mathrm{OTf})_{3}$ showed better result with the less reactive methyl-substituted cyclopropyl ketone.

Scheme 30. Asymmetric ring-opening/cyclization/retroMannich reaction of $D-A$ cyclopropanes with aryl 1,2diamines reported by Feng and co-workers. ${ }^{76}$

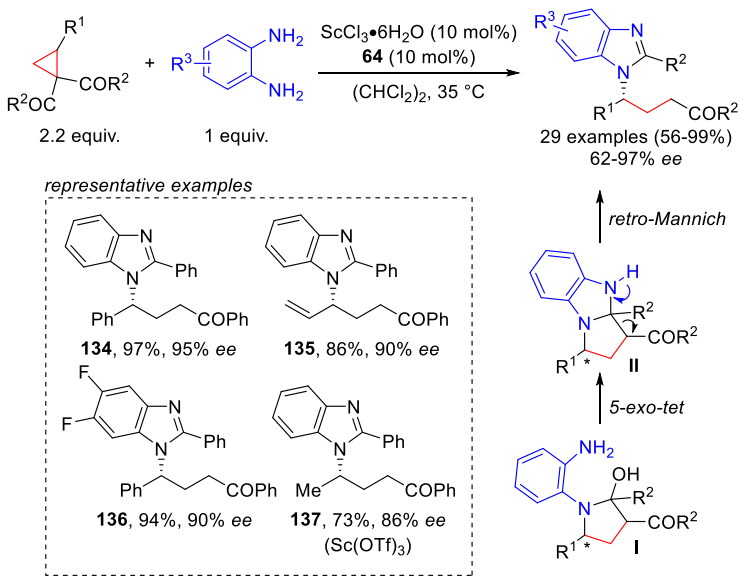

\subsubsection{Via enamine catalysis}

The synthesis of enantioenriched pyrrolo[1,2-a]quinolines was described by Vicario and co-workers through a domino cyclopropane ring opening/aza-Michael/aldol condensation sequence followed by acid-promoted lactamization (Scheme 31). ${ }^{77}$ Activation of the cyclopropylacetaldehyde by silylated prolinol 77 produces a D-A cyclopropane I that undergoes a ring-opening reaction. The formed $\alpha, \beta$-unsaturated iminium ion II then reacts in a domino process with $o$-aminobenzaldehyde (138) with transfer of chirality from the amine catalyst. Dihydroquinolines such as 139-141 were produced in high yields and enantioselectivities. The corresponding pyrroloquinolines were also synthesized using a one-pot procedure adding a lactamization step to the domino sequence.
Scheme 31. Enantioselective synthesis of pyrroloquinolines from cyclopropylacetaldehydes via enamine catalysis reported by Vicario and co-workers. ${ }^{77}$

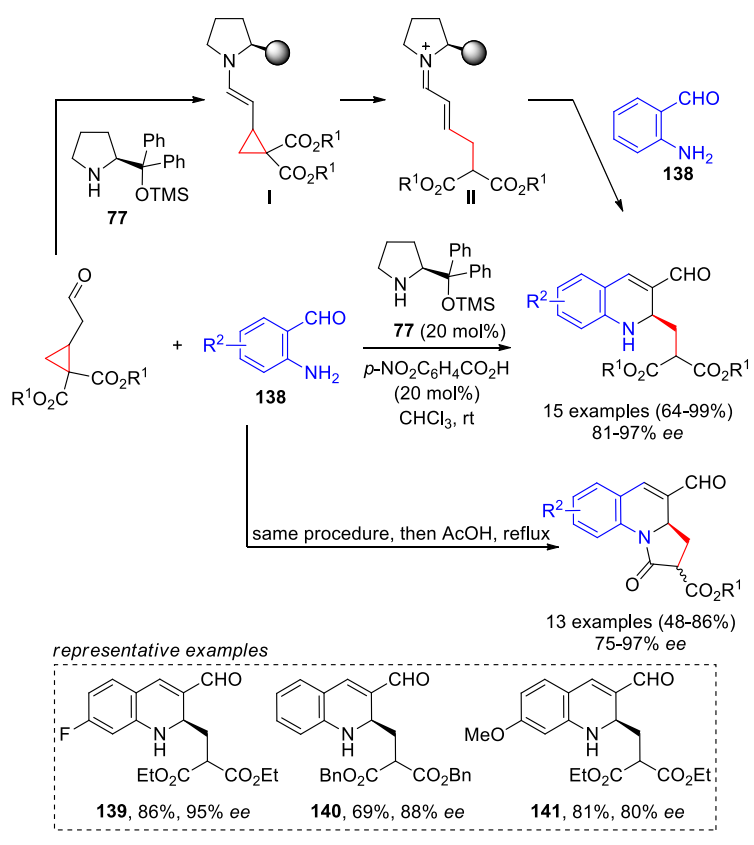

\subsubsection{Other}

$\mathrm{Gu}$ and co-workers reported the synthesis of enantioenriched $\gamma$ lactones from racemic $\beta$-azidocyclopropane carboxylates through a catalytic asymmetric transfer hydrogenation (Scheme 32) ${ }^{78}$ Mechanistically, reduction of the azido group is followed by a ring-opening reaction to give an oxo-ester after hydrolysis of the zwitterionic intermediate. Asymmetric hydrogenation of the oxo-ester then provides an enantioenriched $\gamma$ hydroxybutyrate, which is converted into the corresponding $\gamma$ lactone under acidic conditions in a one-pot process. Ru catalyst 142 gave the desired $\gamma$-lactones with good yields and high levels of enantioselectivity in the presence of a $\mathrm{HCO}_{2} \mathrm{H} / \mathrm{Et}_{3} \mathrm{~N}$ mixture as reductant. Low to moderate yields were obtained depending on the electronic properties of the aryl substituent with good ee values except for aryl groups bearing electron-rich substituents.

Scheme 32. Asymmetric transfer hydrogenation of racemic $\beta$-azidocyclopropanes reported by $\mathrm{Gu}$ and co-workers. ${ }^{78}$

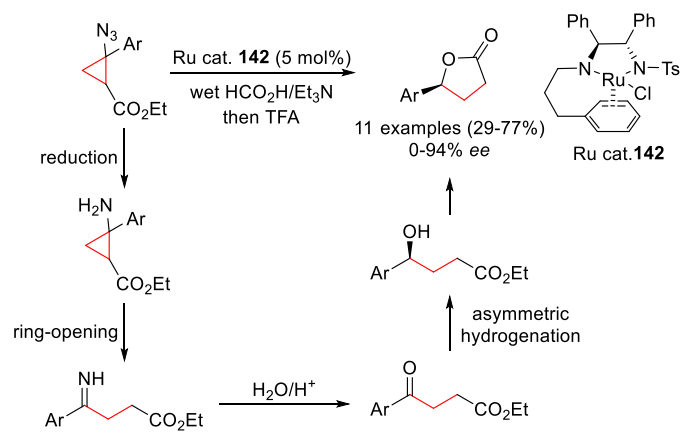




\section{Acceptor-activated cyclopropanes}

Compared to D-A cyclopropanes, acceptor-activated cyclopropanes often require the formation of a more reactive intermediate (e.g. iminium or ketyl radical) to promote a ringopening reaction. Radical methodologies using photoredox catalysis have been mostly used for annulation processes, whereas iminium catalysis from cyclopropyl carbaldehydes is the most common approach for simple ring-opening reactions. Nevertheless, transition metal based activation by oxidative addition, simple Lewis acid activation or base catalysis have also been reported. Concerning types of transformations, $(3+2)$ annulation reactions and 1,3-difunctionalisations have been mostly studied.

\subsection{Annulation reactions}

The activation of cyclopropane diesters by a chiral nickel Lewis acid for the enantioselective $(3+3)$ annulation with nitrones was described by Sibi and co-workers as early as 2005 (Scheme 33).$^{79}$ Initially, ytterbium triflate and various pyBOX ligands were screened but the tetrahydro-1,2-oxazine products were obtained in low enantiomeric excess. Finally, the chiral ligand 143 developed by Kanemasa ${ }^{80}$ was efficient with nickel perchlorate as the Lewis acid. With the optimized conditions, a variety of tetrahydro-1,2-oxazines such as 144-147 were obtained from EWG-activated substituted cyclopropanes with excellent yields and high enantioselectivities. However, very low diasteroselectivities were observed when different substituents $\left(\mathrm{R}^{1} \neq \mathrm{R}^{2}\right)$ were present on the cyclopropane (products such as 147).

Scheme 33. Enantioselectective $(3+3)$ annulation of nitrones with cyclopropane diesters reported by Sibi and coworkers. $^{79}$

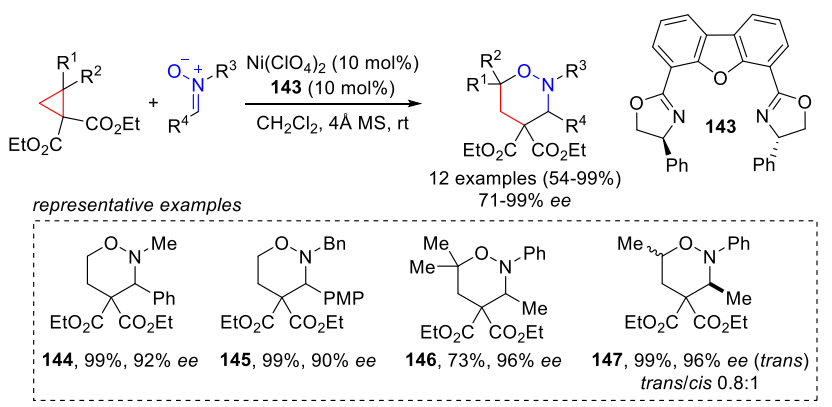

In the example of Sibi, the coordination of the nickel catalyst with the diester group is enough to activate the cyclopropane towards a nucleophilic attack. However, cyclopropanes lacking the diester group cannot be activated by a Lewis acid only. Therefore, this approach remains limited to very few acceptoronly substituted cyclopropanes. In the following examples, transition metal based redox activation and photoredox catalysis were used in $(3+2)$ annulations of cyclopropyl amides and cyclopropyl ketones with unsaturated carbon partners.

Ye and co-workers reported a $\mathrm{Ni}-\mathrm{Al}$ bimetallic catalyst enabling the enantioselective $(3+2)$ annulation of cyclopropyl carboxamides with alkynes based on transition metal oxidative addition (Scheme 34). ${ }^{81}$ Initially, the reaction was examined in a racemic version. During the optimization, the phosphine oxide $\mathrm{Ph}_{2} \mathrm{P}(\mathrm{O}) \mathrm{H}$ was identified as an excellent ligand and high yields were obtained by using additional $\mathrm{PPh}_{3}$. The combination of $\mathrm{AlMe}_{3}$ and nickel with the phosphine ligand is believed to generate a bimetallic species I that may be involved in the activation of the cyclopropane via oxidative addition and stabilization of the in situ formed nickellacycle II. The asymmetric version was successfully performed with a taddolderived chiral phosphine oxide ligand 148. The use of different additional phosphine ligands was required to obtain cyclopentenyl carboxamides such as 149-151 in good yields as well as good ee values. Limitations occurred when dialkylalkynes and unsymmetrical alkynes were used, leading to low yields or low enantiomeric excess for products such as 152. A detrimental effect on the enantioselectivity was also observed when the pyrrolidinyl moiety was replaced by other amine groups (product $\mathbf{1 5 3}$ ).

Scheme 34. Ni-Al bimetallic catalyzed enantioselective $(3+2)$ annulation of cyclopropyl carboxamides with alkynes reported by Ye and co-workers. ${ }^{81}$

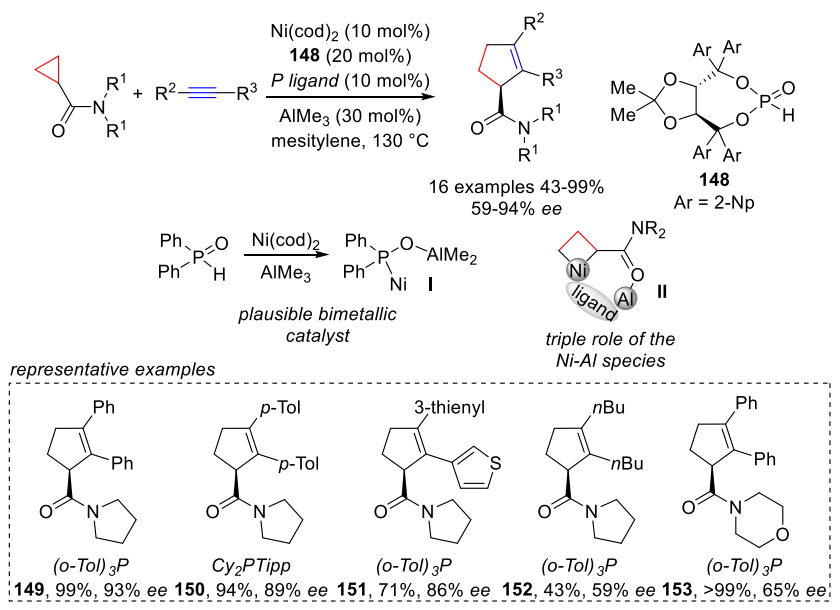

In 2016, the group of Yoon described an enantioselective $(3+2)$ photoannulation of aryl cyclopropyl ketones with alkenes using a dual catalyst system based on photoredox/Lewis acid activation (Scheme 35). ${ }^{82}$ This report is an asymmetric intermolecular version of their previously reported intramolecular photocatalytic $(3+2)$ annulation in which the activation of an aryl cyclopropyl ketone by a Lewis acid allows its one electron reduction to form a ketyl radical. ${ }^{83}$ Ring fragmentation of the ketyl radical then provides an enolate radical intermediate that adds onto the $\pi$-bond, followed by a stereocontrolled radical cyclization to give optically active cyclopentanes. With a ruthenium photocatalyst and $\mathrm{Gd}(\mathrm{III})$ pyBOX (154) as Lewis acid, electronically diverse styrene derivatives and dienes gave cyclopentanes such as $\mathbf{1 5 5 - 1 5 8}$ with good yields and excellent enantioselectivities, but low diastereoselectivities. In contrast, aliphatic alkenes and internal olefins were unreactive. Concerning the substituent at the 2position $\left(\mathrm{R}^{1}\right)$, esters and ketones were well tolerated, but a methyl substituent had a detrimental effect on the yield and the enantioselectivity (product 159). 
Scheme 35. Enantioselective (3+2) photoannulation of aryl cyclopropyl ketones with styrene derivatives reported by Yoon and co-workers. ${ }^{82}$

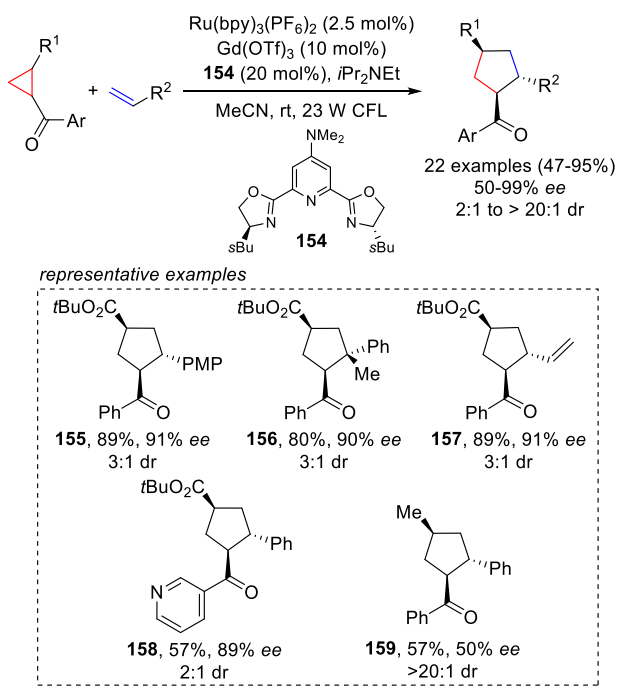

Later, Meggers and co-workers developed an asymmetric $(3+2)$ photoannulation of cyclopropanes with alkenes catalyzed by a single chiral-at-metal rhodium complex 160 (Scheme 36). ${ }^{84}$ Here, the rhodium catalyst plays a dual role: Lewis acid coordination lowers the reduction potential of the cyclopropane and enables a direct photoexcitation of the visible-lightabsorbing catalyst-substrate complex. The corresponding excited state of this complex is ultimately reduced into an enolate radical intermediate promoting the radical process. Chiral cyclopentanes were produced in excellent yields and very high enantioselectivities. Diastereoselectivities were also high except for cyclopropanes bearing two different substituents $\left(R^{1} \neq R^{2}\right)$. Various Michael acceptors (products such as 161-163) and styrenes (products such as 164) were suitable substrates for the $(3+2)$ annulation with substituted cyclopropanes. Furthermore, cyclopentanes containing an allcarbon quaternary stereocenter were also produced with excellent yields and enantioselectivities (products 162 and 164). The scope was extended to alkynes furnishing enantioenriched cyclopentenes such as $\mathbf{1 6 5 - 1 6 8}$ with high yields and enantioselectivities.
Scheme 36. Rhodium-catalysed asymmetric (3+2) photoannulation of cyclopropanes with alkenes and alkynes reported by Meggers and co-workers. ${ }^{84}$

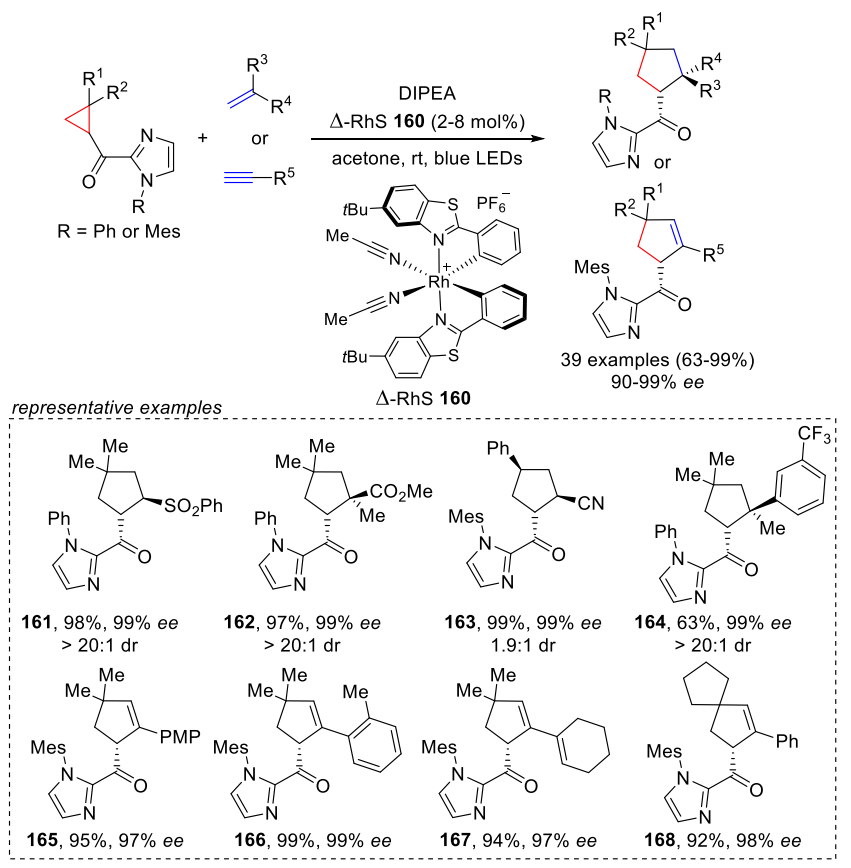

Stereoselective radical $(3+2)$ annulations were also studied by Lin and co-workers based on a radical redox relay strategy using Ti complexes (Scheme 37). ${ }^{85}$ This strategy was initially reported through a formal $(3+2)$ annulation of $N$-acylaziridines and alkenes to provide substituted pyrrolidine derivatives ${ }^{86} \mathrm{By}$ analogy, cyclopropyl ketones were supposed to be suitable substrates in these tandem radical redox processes promoted by $\mathrm{Ti}^{\mathrm{III}}$ complexes. Indeed, the stepwise $(3+2)$ annulation of cyclopropyl ketones with alkenes was successfully developed in an enantioselective manner using chiral Ti(salen) complex 169. Substituted cyclopentanes such as 170-172 were obtained in high yields and generally excellent diastereoselectivities and enantioselectivities from a variety of styrene derivatives. Furthermore, 1,1-disustituted alkenes were also suitable substrates, leading to the formation of a quaternary stereocenter (products such as 173). However, the cyclopropane scope was mostly limited to 2,2-dimethylcyclopropyl phenyl ketone. Indeed, methyl dimethylcyclopropyl ketone provided cyclopentane $\mathbf{1 7 4}$ in good yield but very low enantiomeric excess. The enantioselectivity was also found to decrease considerably with electron-deficient alkenes (e.g. vinyl sulfone, product 175). 
Scheme 37. Stereoselective radical $(3+2)$ annulation of cyclopropyl ketones with alkenes catalyzed by chiral Ti(salen) complex 169 reported by Lin and co-workers. ${ }^{85}$
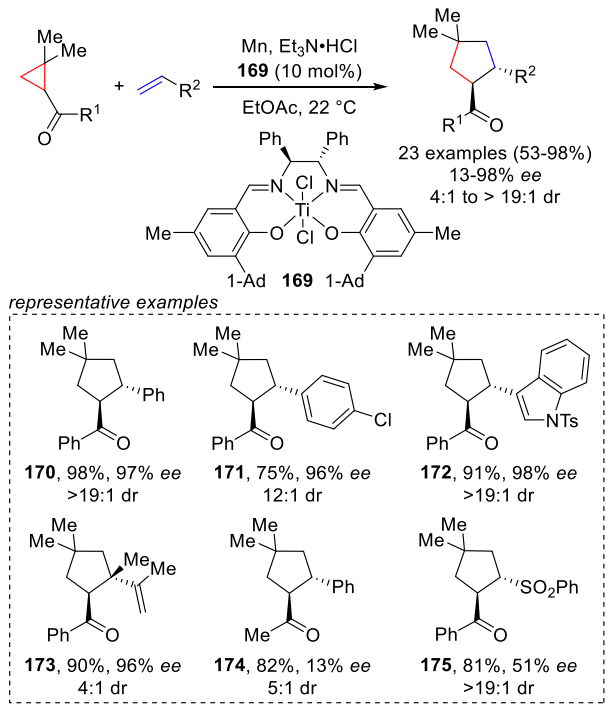

\subsection{Ring-opening reactions}

Ring-opening reactions of acceptor-activated cyclopropanes have been accomplished through the desymmetrization of spirocyclopropanes and cyclopropyl carbaldehydes via nucleophile catalysis and enamine catalysis respectively.

\subsubsection{Via nucleophile/base catalysis}

An enantioselective cob(I)alamin-catalysed desymmetrization of spiro-cyclopropanes was reported by Scheffold and Troxler through nucleophilic catalysis (Scheme 38). ${ }^{87}$ Enantioenriched $(R)$-Meldrum's acid derivatives with a cycloalk-2-enyl moiety were formed through the nucleophilic attack of the chiral cobalamin (Vitamine $\mathrm{B}_{12}$ ) to give intermediate $\mathbf{I}$, followed by reductive elimination of the cobalt(III) intermediate. This unique way to desymmetrize meso-cyclopropanes has however not found broader application, as only two examples were reported.

Scheme 38. Cob(I)alamine-catalyzed desymmetrization of spiro-cyclopropanes reported by Scheffold and Troxler. ${ }^{87}$

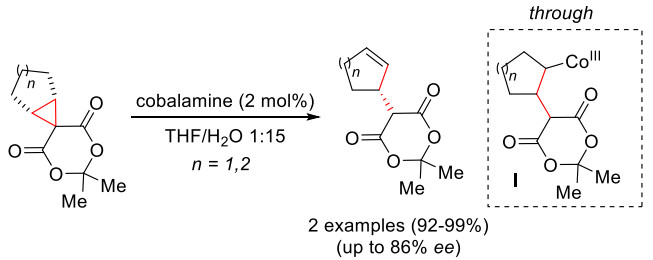

Müller and Riegert described the desymmetrization of spirocyclopropane 176 through the nucleophilic addition of thiophenol in the presence of cinchonidine (Scheme 39). ${ }^{88}$ Modest $e e$ values were obtained. The formation of a chiral ion pair by deprotonation of thiophenol with cinchonidine was proposed to rationalize these results.
Scheme 39. Desymmetrization of spiro-cyclopropane 176 by ring-opening with thiols in presence of cinchonidine reported by Müller and Riegert. ${ }^{88}$

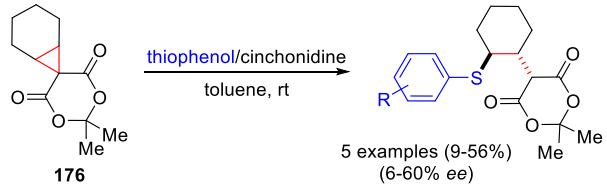

\subsubsection{Via enamine catalysis}

The desymmetrization of meso-cyclopropyl carbaldehydes was performed via iminium-enamine catalysis often leading to 1,3difunctionalized acyclic products. In the presence of a chiral amine catalyst, the formation of an iminium facilitates cyclopropane ring-opening, generating an enamine that is ultimately intercepted by an electrophile.

Gilmour and Sparr described first the desymmetrization of meso-cyclopropanecarbaldehydes through an amine-catalyzed enantioselective synthesis of 1,3-dichlorides (Scheme 40). ${ }^{89}$ Activation of the cyclopropylcarbaldehyde with MacMillantype imidazolidinone $\mathbf{1 7 7}$ via the transient cyclopropyl iminium ion allowed the nucleophilic addition of a chlorine atom in an enantioselective manner. The formed enamine $\mathbf{I}$ reacted further with an electrophilic chlorinating reagent to give $\alpha-\gamma$ dichlorinated aldehydes such as 178-181 with good yields and good ee values.

Scheme 40. Enantioselective 1,3-difunctionalization of meso-cyclopropanes through iminium-enamine catalysis reported by Gilmour and Sparr. ${ }^{89}$

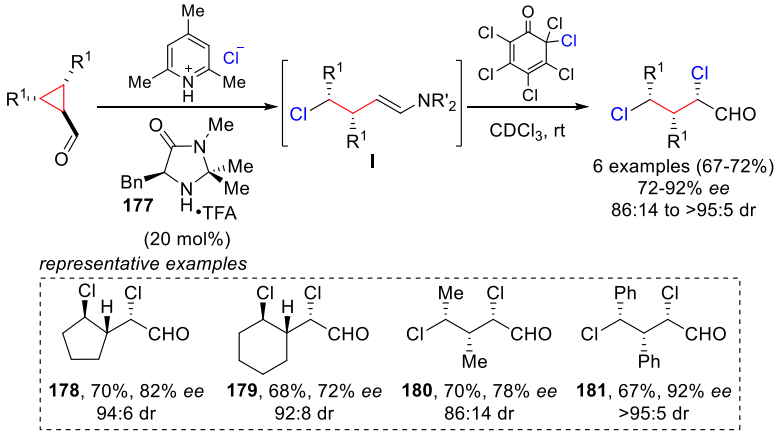

Using this strategy, Werz and co-workers developed an enantioselective 1,3-chlorochalcogenation of cyclopropyl carbaldehydes (Scheme 41). ${ }^{90}$ Structural modifications of the chiral imidazolidinone organocatalyst were crucial to improve the enantioselectivity. The best results were obtained with naphthyl-substituted imidazolidinone 182. Various substituted aryl sulfenyl chlorides as well as sterically demanding alkyl sulfenyl chlorides gave 1,3-chlorosulfenation products such as 183-185 with moderate yields and moderate to good enantiomeric excesses and diastereoselectivities. Limitations occurred when a protected pyrrolidine-derived cyclopropyl carbaldehyde was used, leading to no enantioselectivity for product $\mathbf{1 8 6}$. 
Scheme 41. Enantioselective 1,3-chlorochalcogenation of cyclopropyl carbaldehydes reported by Werz and coworkers. ${ }^{90}$

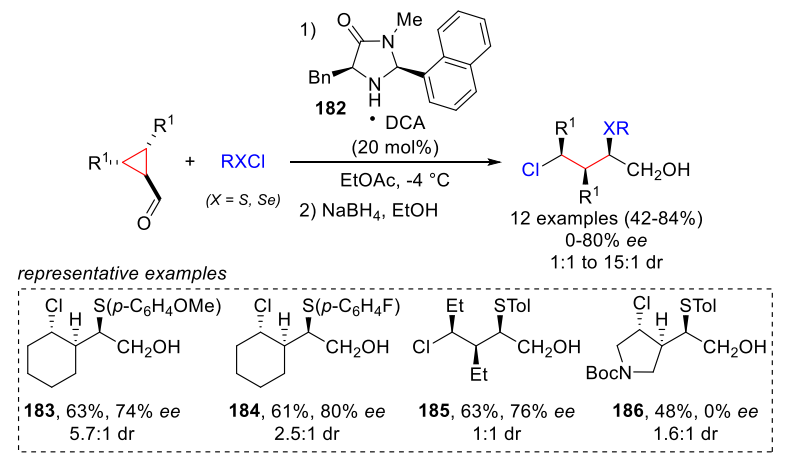

Vicario and co-workers then reported a desymmetrization of meso-formylcyclopropanes using carboxylic acids as nucleophiles (Scheme 42). ${ }^{91}$ Whereas the MacMillan-type imidazolidinones have shown efficient enantioinduction in the two previous examples, proline-derived catalyst 187 containing a bulky silyl group $\left(\mathrm{SiPh}_{2} \mathrm{Me}\right)$ improved considerably the enantioselectivity in this case. $\gamma$-Acyloxy-substituted aldehydes such as 188-191 were obtained in moderate to high yields and enantioselectivities starting from various carboxylic acids (benzoic or aliphatic) and monocyclic or bicyclic formylcyclopropanes.

Scheme 42. Enantioselective ring opening of formylcyclopropanes with carboxylic acids under iminium ion catalysis reported by Vicario and co-workers. ${ }^{91}$

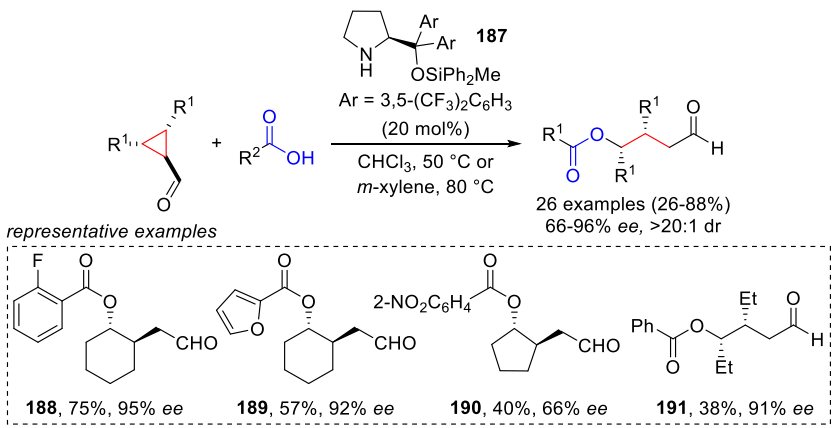

\section{Donor-activated cyclopropanes}

Donor-only-activated cyclopropanes have been less described in stereoselective ring-opening reactions. In particular, oxidative ring-opening and electrophilic addition strategies using metal and organocatalysis were applied to aromatic substituted cyclopropanes, cyclopropanols and aminocyclopropanes owing to their electron-rich character.

With regards to annulation reactions, the group of Melchiorre described an organocatalyzed enantioselective photochemical cascade process converting racemic cyclopropanols and $\alpha, \beta$ unsaturated aldehydes into cyclopentanols (Scheme 43). ${ }^{92}$ In contrast to iminium-based methods seen so far, the annulation partner is activated instead of the cyclopropane. After condensation of the chiral amine catalyst 192 with cinnamaldehyde, selective excitation of the chiral iminium ion gives electronically excited state $\mathbf{I}^{*}$, which acts as a strong oxidant towards the cyclopropanol. Upon SET (Single-Electron Transfer) oxidation, the cyclopropanol is converted into an unstable oxycyclopropyl radical cation, which undergoes a fast ring-opening leading to non-chiral $\beta$-keto radical cation II. Radical coupling of the latter with the chiral $\beta$-enaminyl radical III sets the first stereogenic center. Finally, aldol cyclization of IV affords the cyclopentanol. Complex cyclopentanol products with three stereocenters such as 193-197 were obtained in good yields and excellent $e e$ and dr from cyclopropanols bearing alkyl, benzyl and heterocyclic substituents. With respect to the $\alpha, \beta$-unsaturated aldehydes, electronically-diverse $\beta$-aromatic moieties were well tolerated but $\beta$-alkyl fragments were unreactive. The high stereoselectivity observed in this transformation was attributed to the good facial control in the recombination of radicals II and III controlled by chiral catalyst 192.

Scheme 43. Enantioselective photochemical organocascade converting cyclopropanols into cyclopentanols reported by Melchiorre and co-workers. ${ }^{92}$

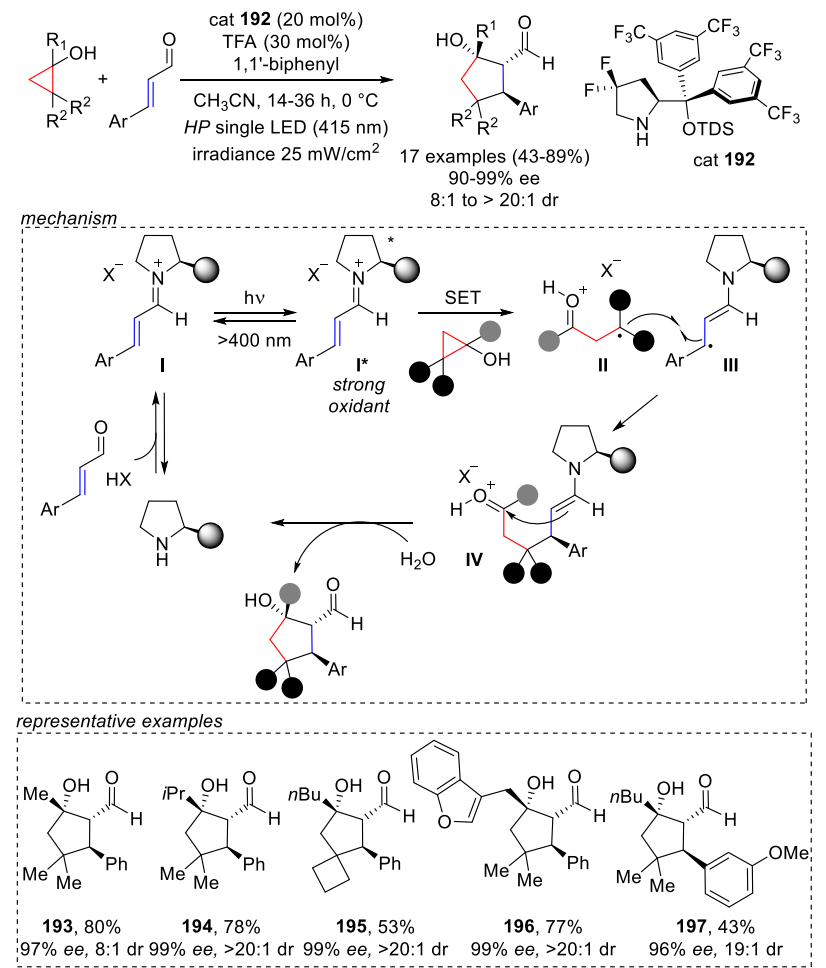

In ring-opening reactions, donor-activated cyclopropanes have been used after oxidation as formal 1,3-dielectrophilic carbon intermediates, so that two nucleophiles can be incorporated. Oxidation of electron-rich cyclopropanes leads to ring fragmentation to form a radical cation, followed by addition of a nucleophile. The newly formed radical then can be further functionalized either through a radical trapping or through transition metal catalysis allowing the incorporation of a second nucleophile. Recently, a copper-catalyzed enantioselective aminocyanation of arylcyclopropanes was reported by Zhang and co-workers (Scheme 44). ${ }^{93}$ Mechanistically, the reaction occurs through a cyclopropyl radical cation intermediate leading to a stable benzylic radical after nucleophilic addition of the amine. The benzylic radical then recombines with the chiral copper catalyst to give the enantioenriched $\gamma$ aminonitriles after reductive elimination. The scope was carried out with the BOX ligand 198. $\gamma$-Aminonitriles such as 199-205 
were obtained in general with excellent yields and enantioselectivities from various substituted arylcyclopropanes.

Scheme 44. Copper-catalyzed enantioselective aminocyanation of arylcyclopropanes reported by Zhang and co-workers. ${ }^{93}$
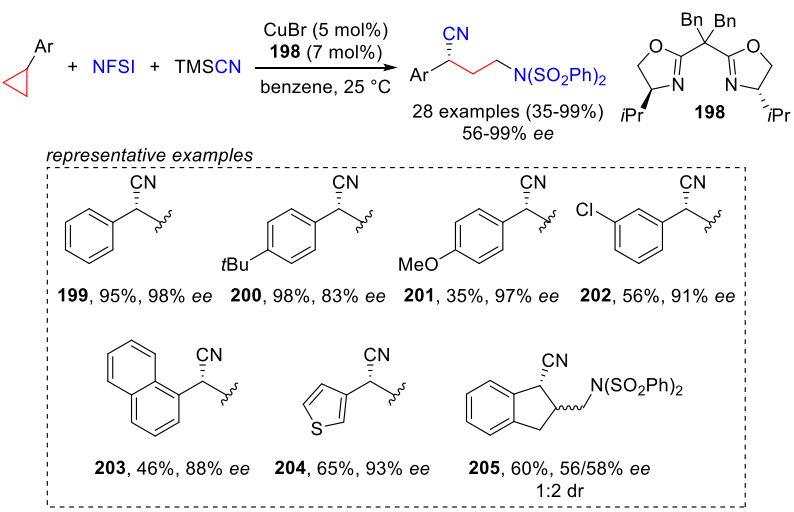

An enantioselective difluorination of arylcyclopropanes was also attempted by Jacobsen and co-workers through the 1,3oxidation of cyclopropanes using a chiral hypervalent iodine catalyst, but very low levels of enantiocontrol were observed. ${ }^{94}$

The use of cyclopropanes as 1,3-dielectrophilic carbon intermediates was also described by our group in the ringopening reaction of protected aminocyclopropanes leading to 1,3-difunctionalized propylamines. ${ }^{95}$ Although this reaction was studied in its racemic version, a proof of concept for asymmetric induction was shown using chiral phosphoric acid 207 as catalyst for the ring-opening of aminocyclopropane 206 to give $N, O$-acetal 208 (Scheme 45). In this case, in situ formation of the iodo imine followed by acid-catalyzed enantioselective addition of methanol was proposed.

Scheme 45. Enantioselective synthesis of 1,3difunctionalized propylamine 208 through the ring-opening of aminocyclopropane 206 reported by Waser and coworkers. ${ }^{95}$

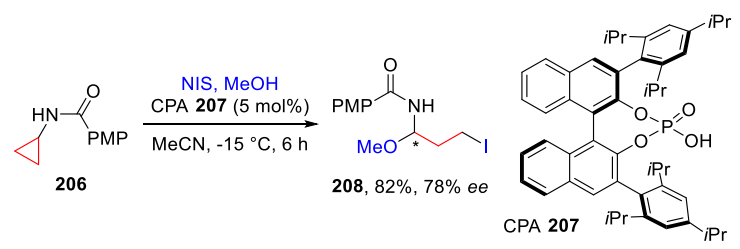

Cyclopropanols were also used as masked homoenolates in the stereoselective addition onto unsaturated systems. Yoshikai and co-workers described a cobalt-catalyzed enantioselective and chemodivergent addition of cyclopropanols to oxabicyclic alkenes (Scheme 46). ${ }^{96}$ In presence of DABCO (1,4Diazabicyclo[2.2.2] octane), a combination of the cyclopropanol and the Co(II) species produced a cobalt(II) cyclopropoxide, which delivers the corresponding cobalt(II) homoenolate after ring opening. Then, the reaction proceeds through the carbocobaltation of this homoenolate to the oxabicyclic alkene leading to an alkylcobalt species I. From this common intermediate two pathways were possible in dependence of the counterion on the cobalt catalyst. Alkylated ring-opened products were formed with cobalt(II) chloride and chiral diphosphine 209, whereas hydroalkylated products were obtained with cobalt(II) acetate and the same diphosphine ligand 209 in the presence of methanol. Various aryl cyclopropanols participated in both processes. Electron withdrawing- as well as electron donating-substituents on the aryl moiety were well tolerated, furnishing products such as 210-213 or 215-217 in good yields and enantioselectivities. Notably, azabenzonorbornadiene also gave hydroalkylation product 218. The desired products 214 and 219 were also obtained with phenyl-ethyl- and benzyl- substituted cyclopropanols.

Scheme 46. Cobalt-catalyzed enantioselective and chemodivergent addition of cyclopropanols to oxabicyclic alkenes reported by Yoshikai and co-workers. ${ }^{96}$
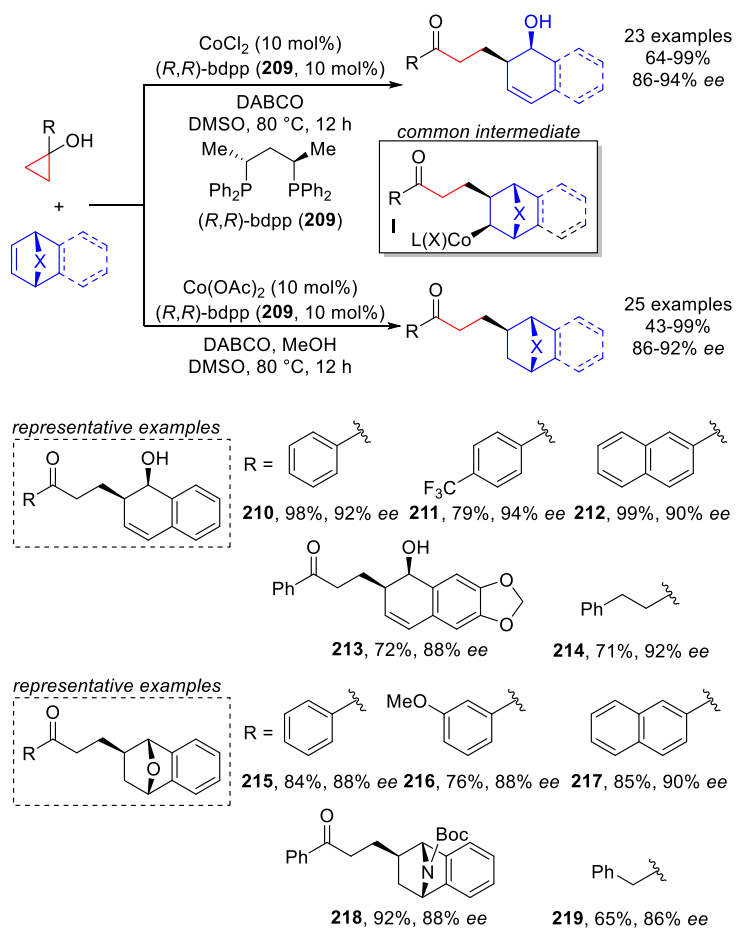

\section{Vinylcyclopropanes}

Vinylcyclopropanes (VCPs) stand as a special type of donoracceptor cyclopropanes. In the presence of transition metals, the vinyl substituent serves as a coordinating group, facilitating the opening of the three-membered ring to give different types of intermediates, such as $\pi$-allyl complexes or carbocations. Successful applications in the field of asymmetric catalysis were reported with palladium, rhodium, iridium and gold complexes, allowing the development of annulations, ring expansions, as well as electrophilic and nucleophilic ring openings of VCPs. The ring opening of VCPs can also be triggered by the addition of an appropriate radical onto its vinyl substituent. Using thiyl or trifluoromethyl radicals, enantioselective annulations and a 1,5-difunctionalization were reported.

\section{1. $\quad(3+2)$ Annulations}

The $(3+2)$ annulation is the most studied transformation involving VCPs in the context of asymmetric catalysis. This type of transformations provides a straightforward access to highly functionalized and stereodefined five membered rings. 


\subsubsection{Palladium catalysis}

The coordination of a palladium catalyst to the vinyl substituent of a VCP activated by one or several electron-withdrawing groups triggers a ring opening leading to a zwitterionic $\pi$-allyl complex, that can then react with an appropriate dipolarophile. In 2011, building upon the seminal work of Tsuji and coworkers, ${ }^{97}$ Trost and co-workers described the first enantioselective synthesis of substituted cyclopentanes by a palladium-catalyzed $(3+2)$ annulation of VCPs (Scheme 47). ${ }^{98}$ With alkylidene azlactones as acceptors and VCP 220, this method provides an expedient access to enantioenriched amino acid derivatives bearing three stereogenic centers. Cycloadducts such as 222-225 could be obtained in high diastereo- and enantioselectivities by employing the diphenylphosphino benzoic acid (DPPBA) based ligand $(\boldsymbol{S}, \boldsymbol{S})$ 221 combined with a $\operatorname{Pd}(0)$ catalyst. While the transformation tolerated various substitution on the Michael acceptor, the use of bis(2,2,2-trifluoroethyl)malonate VCP $\mathbf{2 2 0}$ was necessary to ensure higher yields and selectivities.

Scheme 47. Palladium-catalyzed asymmetric (3+2) annulation of VCP 220 with alkylidene azlactones reported by Trost and co-workers. ${ }^{98}$

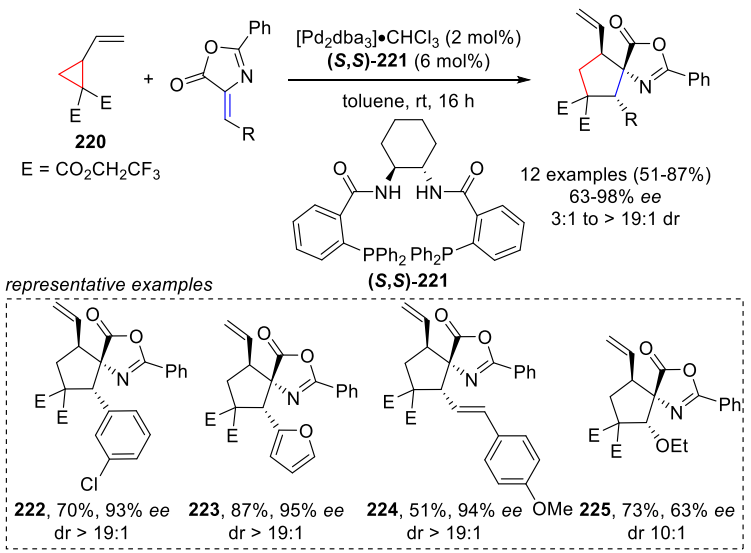

A year later, the same group reported an enantioselective $(3+2)$ annulation between Meldrum's acid substituted alkylidenes and VCP 226 (Scheme 48). ${ }^{99}$ For this new type of two carbon partners, Meldrum's acid-substituted VCP 226 was found to give higher level of diastereo- and enantiocontrol than VCP 220. The best results were obtained with bisphophine 227 as ligand and dioxane as solvent. Under these conditions, a range of aryl-, heteroaryl- and alkynylsubstituted alkylidenes furnished cycloadducts such as 228-230 with good level of enantio- and diastereocontrols. Interestingly, the two Meldrum's acid moieties could be chemoselectively modified to access highly functionalized cyclopentanes. Due to the distance between the chiral pocket around the formed $\pi$-allyl complex and the first bond forming event, the high stereocontrol observed is difficult to rationalize. As shown in scheme 48, Trost and co-workers have proposed an explanation based on the Curtin-Hammett principle: A reversible conjugate addition of zwitterionic intermediate $\mathbf{I}$ on the Michael acceptor results in two diastereomeric complexes II, with one of the two able to undergo a ligand controlled ring closure faster than the other.
Scheme 48. Palladium-catalyzed asymmetric (3+2) annulation of substituted VCPs with Meldrum's acid alkylidenes reported by Trost and co-workers. ${ }^{99}$

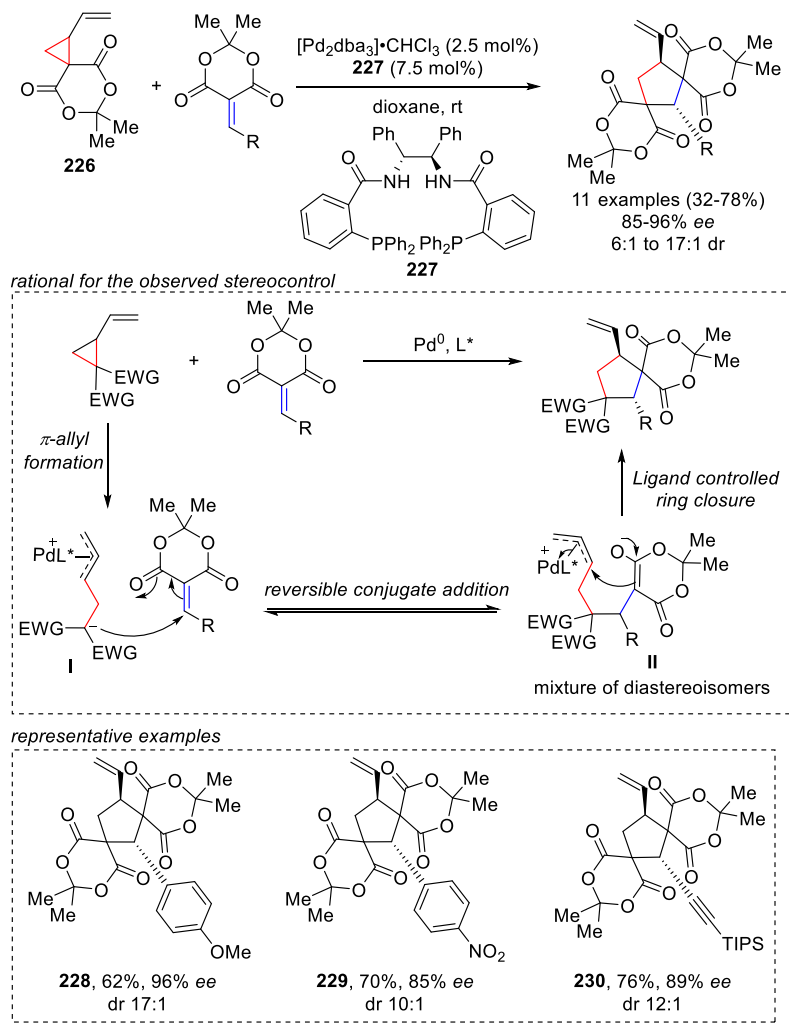

$\beta$, $\gamma$-unsaturated $\alpha$-keto esters were also shown by Shi and coworkers to be suitable dipolarophiles for palladium-catalyzed asymmetric $(3+2)$ annulations with VCPs (Scheme 49). ${ }^{100}$ For this transformation, they reported a new catalytic system involving chiral N,P ligand $\mathbf{2 3 2}$ with a 1,1'-binaphthalene scaffold. A variety of electronically and sterically different $\beta, \gamma-$ unsaturated $\alpha$-keto esters afforded enantioenriched cyclopentanes such as 233-237, with a decrease in yield and enantioselectivity observed when $\mathrm{R}^{2}$ was an electron poor aromatic substituent (product 234). They further reported the use of $\alpha, \beta$-unsaturated $N$-acyl pyrrole 238 and dicyanosubstituted VCP $\mathbf{2 4 0}$ to give the corresponding cyclopentanes 239 and 242 in good to excellent yields and enantioselectivities. 
Scheme 49. Palladium-catalyzed asymmetric (3+2) annulation of VCPs with $\beta, \gamma$-unsaturated $\alpha$-keto esters and acyl pyrroles reported by Shi and co-workers. ${ }^{100}$

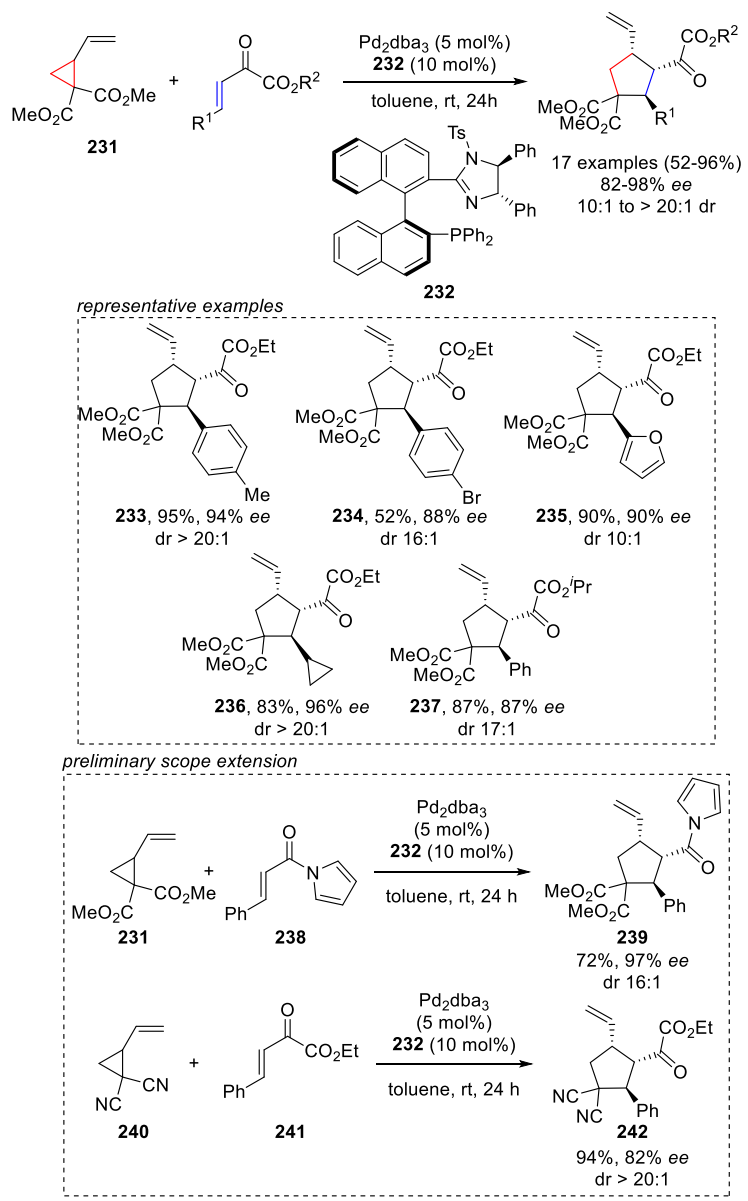

This type of annulation could also be successfully extended to $\alpha$-nucleobase substituted acrylates, giving a fast access to enantioenriched carbocyclic nucleoside analogues (Scheme 50). ${ }^{101}$ Employing Trost ligand $(\boldsymbol{R}, \boldsymbol{R})-221$ good enantioselectivities and yields could be obtained albeit with low diastereoselectivities. The latter could not be significantly improved in spite of a thorough screening of substituents on both reaction partners. With VCP 231, various $\alpha$-purine, thymine or uracil ethyl acrylates gave products such as 243-246. In addition, other heterocycles were also tolerated to form products such as 247-249.
Scheme 50. Palladium-catalyzed enantioselective $(3+2)$ annulation of VCP 231 with $\alpha$-heterocycle substituted acrylates reported by Guo and co-workers. ${ }^{101}$

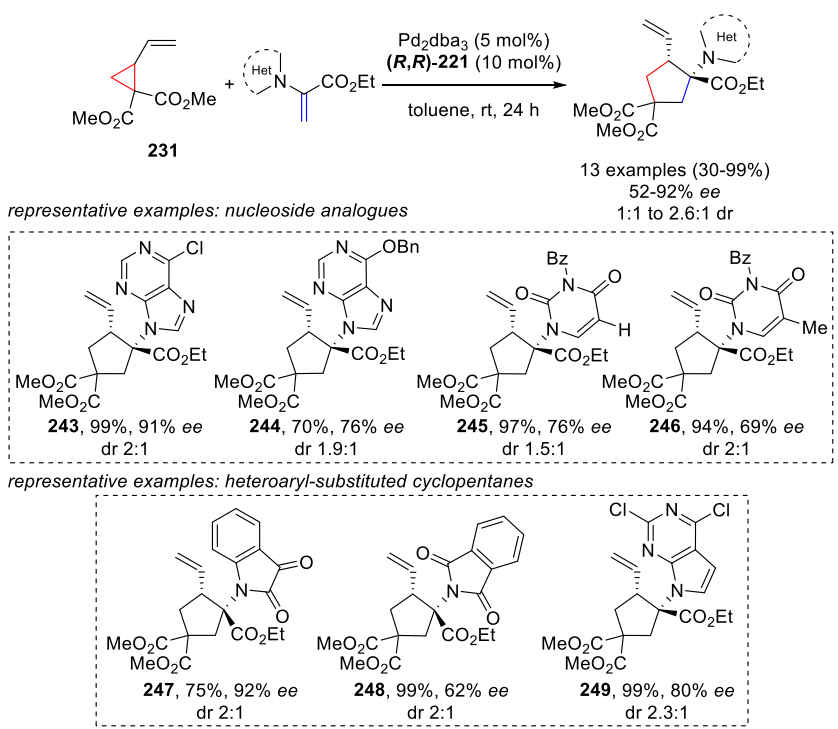

In 2015, two groups reported the enantioselective palladium catalyzed $(3+2)$ annulation of VCPs with nitroolefins to give nitrocyclopentanes, which can be easily converted to cyclopentylamines, an important motif found in various bioactive compounds (Schemes 51 and 52). The group of Q. Z. Liu found vinylcyclopropane dicarbonitrile $\mathbf{2 4 0}$ to be the best partner for this annulation, as for instance diester-substituted VCP 231 led to poor yields and enantioselectivities (Scheme 51). ${ }^{102}$ Performed in the presence of $5 \mathrm{~mol} \%$ of $\mathrm{Pd}(\mathrm{dba})_{2}$ combined with $10 \mathrm{~mol} \%$ of chiral biphosphine $\mathbf{2 5 0}$ as ligand, cycloadducts such as 251-255 were obtained in good yields and enantioselectivities, albeit in low diastereoselectivities. While aryl as well as heteroaryl substituted nitroolefins were generally well tolerated in the transformation, the authors also report one example of an alkyl-substituted nitroolefin, leading to cycloadduct $\mathbf{2 5 5}$ with a slightly lower yield but good enantioselectivity.

Scheme 51. Enantioselective palladium-catalyzed $(3+2)$ annulation of VCP 240 with nitroolefins reported by $Q$. $Z$. Liu and co-workers. ${ }^{102}$

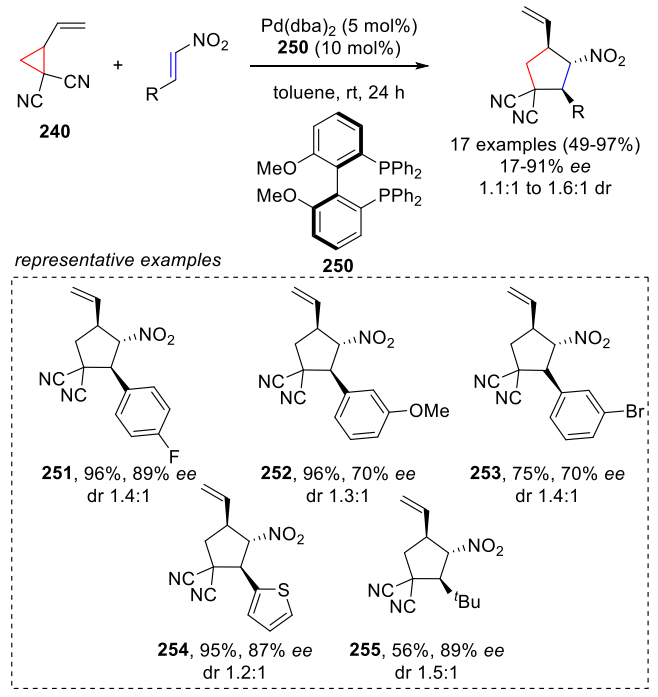


The group of L. Liu independently reported the use of 1,3indanedione substituted VCP 256 in an asymmetric palladiumcatalyzed $(3+2)$ annulation with nitroolefins (Scheme 52). ${ }^{103}$ Proceeding in THF at slightly higher temperature and longer reaction time, this process led to high yields and enantioselectivities for cycloadducts such as 258-262, as well as a slightly improved diastereoselectivity. This was achieved by the use of the bulky and strongly coordinating $N, N$-ligand 257 associated with $\left[\mathrm{Pd}_{2}(\mathrm{dba})_{3}\right]$. Both electron-withdrawing and -donating groups were well tolerated on the aryl moiety of the nitroolefin, as well as heteroaryl and alkyl substituents.

Scheme 52. Palladium-catalyzed asymmetric (3+2) annulation of 1,3-indanedione derived VCP 256 with nitroolefins reported by $\mathrm{L}$. Liu and co-workers. ${ }^{103}$

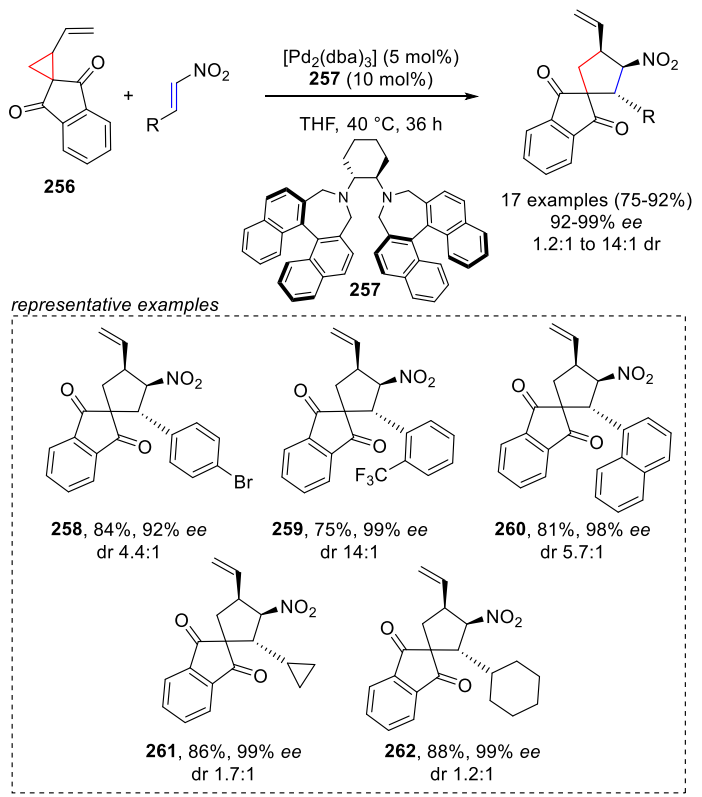

In 2015, Q. Z. Liu and co-workers also reported the use of $\alpha, \beta$ unsaturated imines as dipolarophiles, generated in situ from sulfonyl indoles (Scheme 53). ${ }^{104}$ This palladium-catalyzed (3+2) annulation with VCP 231 led to enantiomerically enriched spiroindolenines. At $10{ }^{\circ} \mathrm{C}$, using $\mathrm{Pd}(\mathrm{dba})_{2}$ and phosphoramidite ligand $\mathbf{2 6 3}$ in THF, various spiroindolenines such as 264-270 could be obtained in good yields and enantioselectivities. Interestingly an excess of VCP 231 was necessary for the reaction to proceed in satisfying yields. It was proposed that the carbanion of the zwitterionic intermediate I formed by oxidative addition with palladium would act as a base to generate in situ the $\alpha, \beta$-unsaturated imine $\mathbf{2 7 2}$ from sulfonyl indole 271, in addition to participating in annulation with 272 to give intermediate II leading to product 273. Therefore, a second equivalent of $\mathbf{2 3 1}$ is needed. This postulate was corroborated by the isolation of compound 275 resulting from the reaction of benzenesulfinate (274) with $\pi$-allyl complex III.
Scheme 53. Palladium-catalyzed enantioselective $(3+2)$ annulation of VCP 231 with in situ generated unsaturated imines reported by $\mathbf{Q}$. Z. Liu and co-workers. ${ }^{104}$

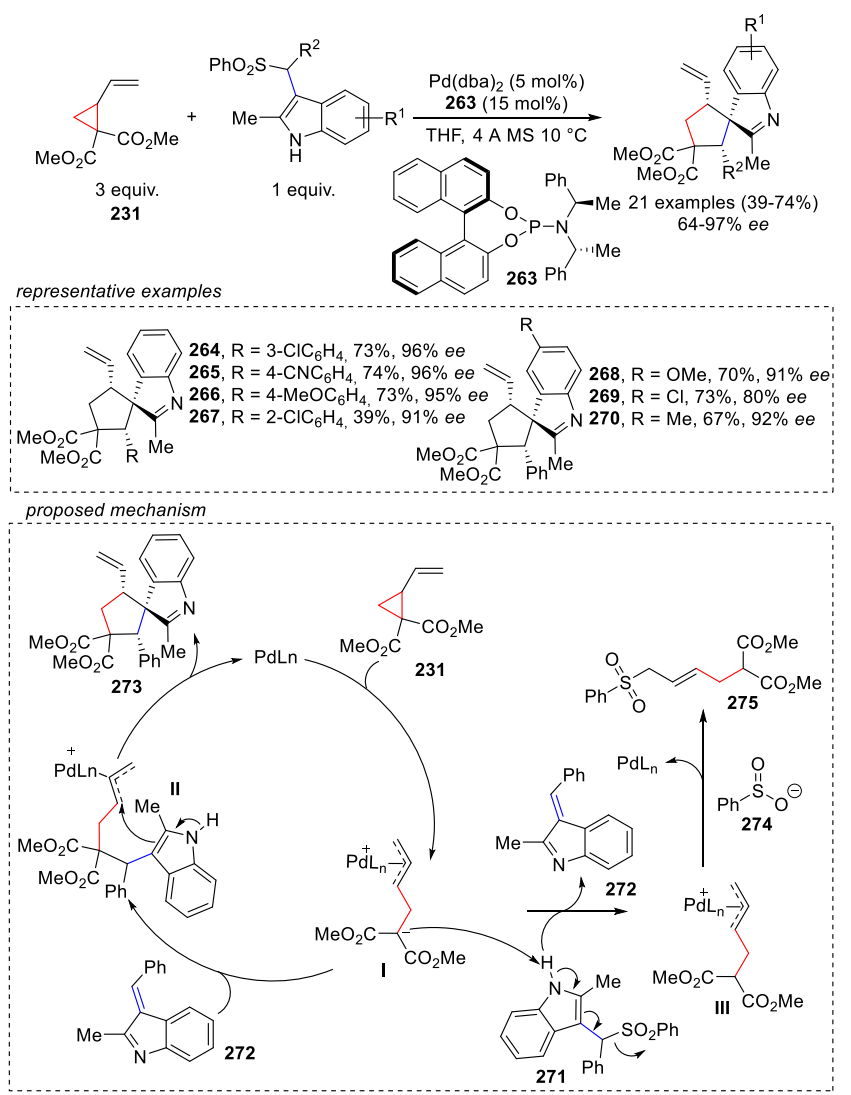

The same group investigated also cyclic 1-azadienes as annulation partners (Scheme 54). ${ }^{105}$ With this time phosphoramidite $\mathbf{2 7 6}$ as ligand, they could access in high yields diverse enantioenriched substituted cyclopentanes such as $\mathbf{2 7 7}$ 283 with moderate to good diastereoisomeric ratios. The 1,2,3oxathiazine 2,2-dioxide motif of the obtained products could be opened by treatment with Red-Al, affording the corresponding products without loss of enantiopurity. 
Scheme 54. Palladium-catalyzed asymmetric (3+2) annulation of VCP 231 with cyclic 1-azadienes reported by Q. Z. Liu and co-workers. ${ }^{105}$

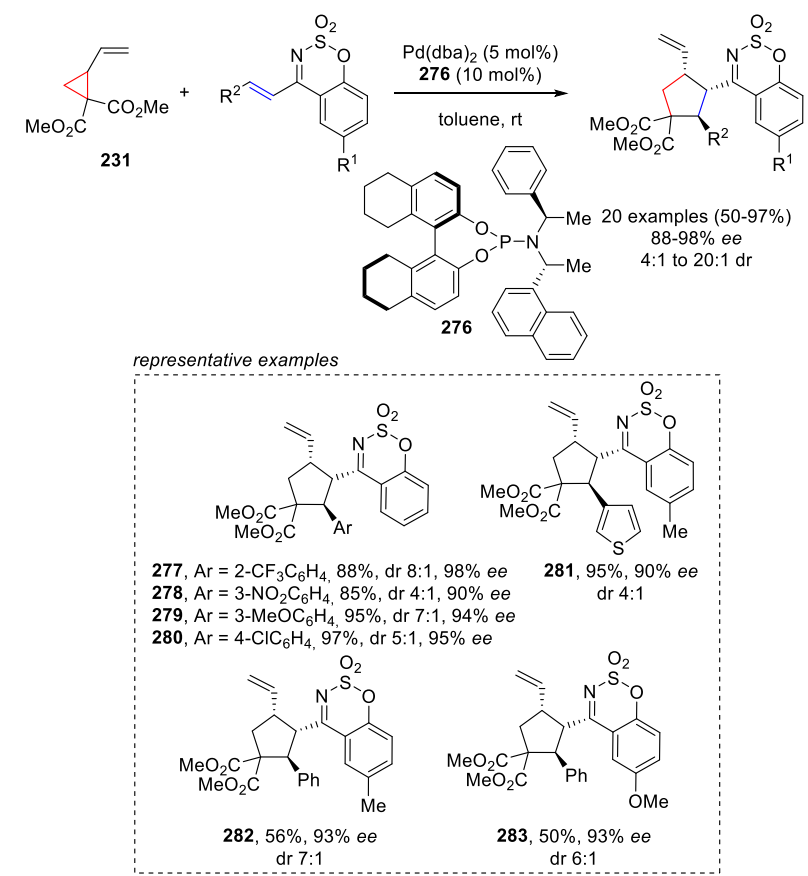

A palladium catalyzed asymmetric $(3+2)$ annulation of VCPs with para-quinone methides was reported by Zhao and coworkers (Scheme 55). ${ }^{106}$ Proceeding through a 1,6-conjugate addition/annulation, this transformation provides an efficient access to enantioenriched (2-oxa)spiro[4.5]decanes such as 285-292, a scaffold found in several bioactive compounds. With chiral biphosphine ligand 284, the highest levels of stereocontrol were obtained for ortho-substituted phenyl groups on the dipolarophile combined with bulky ester groups on the VCP. Different ester groups on the VCP were also tolerated. However, the use of a methyl substituted para-quinone methide led to an important decrease in stereoselectivity.
Scheme 55. Enantioselective palladium-catalyzed $(3+2)$ annulation of VCPs with para-quinone methides reported by Zhao and co-workers. ${ }^{106}$

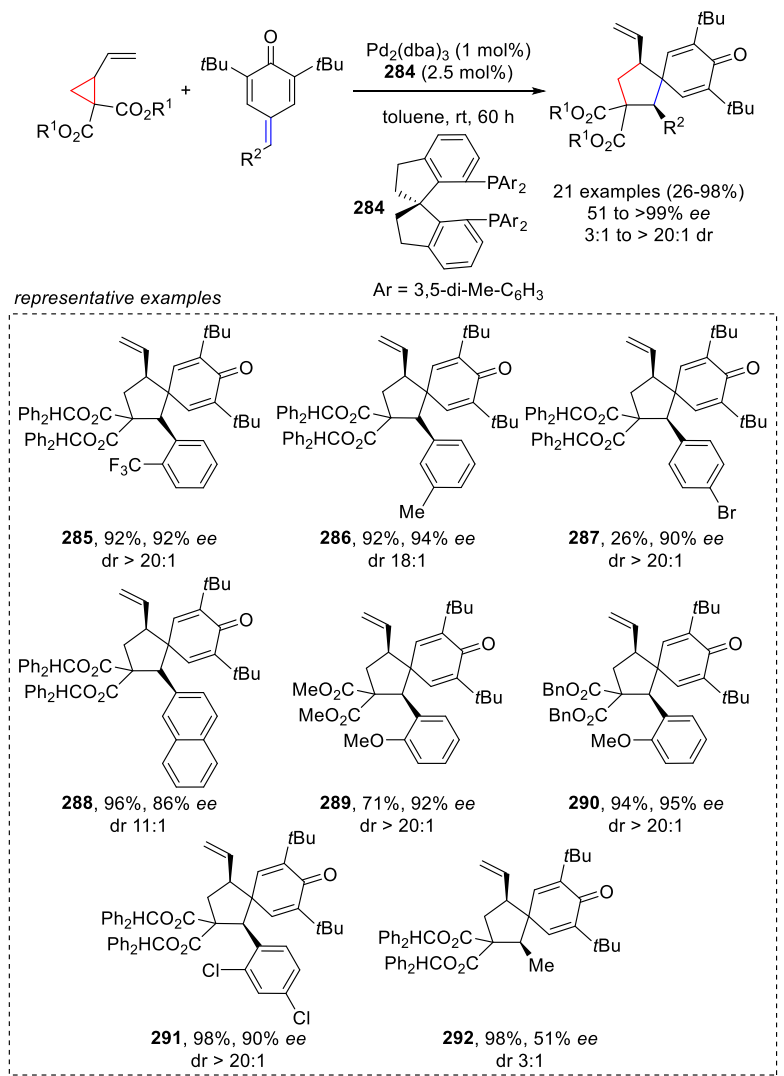

Functionalized cyclopentenes could also be accessed by an asymmetric palladium catalyzed $(3+2)$ annulation between VCP 240 and electron deficient alkynes, as reported by Hou and co-workers (Scheme 56). ${ }^{107}$ Fine-tuning of the ligand, solvent and temperature were required to achieve good results in terms of yields, enantioselectivity and chemoselectivity. Indeed, in addition to the all-carbon cycloadduct (A), a competitive reaction with the ketone of the dipolarophile furnished the corresponding tetrahydrofuran derivative (B). Using Segphos ligand 293, a broad range of alkynyl $\alpha$-ketoesters and alkynyl 1,2-diones gave cyclopentene derivatives such as 294-300 in good yields and enantioselectivities. In the case of a less reactive silyl substituted acrylate, mild heating was required to give cyclopentene 299 in moderate yield. 
Scheme 56. Palladium-catalyzed asymmetric (3+2) annulation of VCP 240 with electron-deficient alkynes reported by Hou and co-workers. ${ }^{107}$

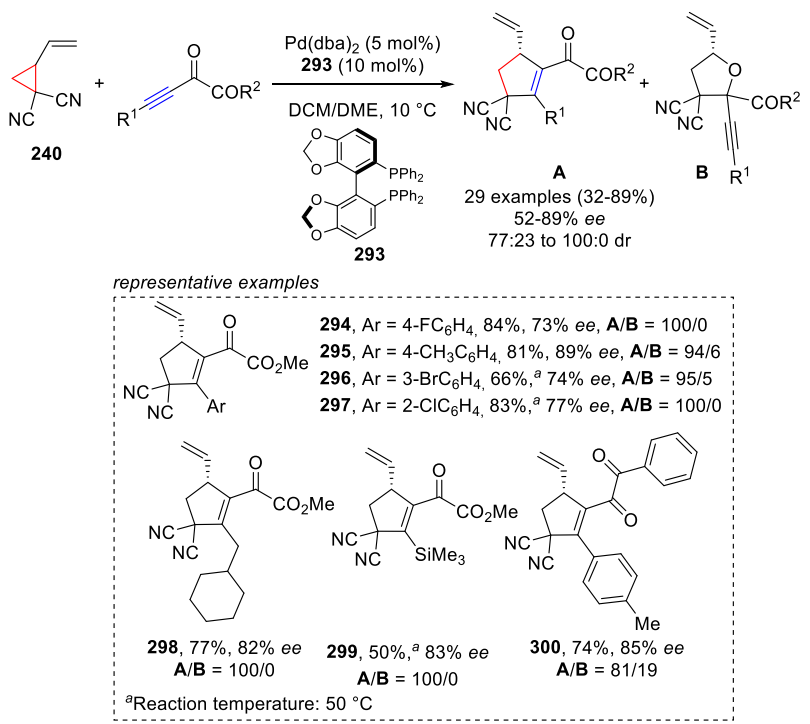

Shi and co-workers applied the annulation process to an enantioselective dearomatization of electron-deficient indoles (Scheme 57). ${ }^{108}$ The use of $\mathrm{Pd}(\mathrm{dba})_{2}$ with phosphoramidite ligand 301 in acetonitrile at $-15{ }^{\circ} \mathrm{C}$ gave a variety of cyclopenta $[b]$ indoline derivatives such as $\mathbf{3 0 2 - 3 0 5}$ in good yields, high enantioselectivities and moderate distereoselectivities. While limited to dicyano VCP 240, a broad range of 3-nitroindoles bearing different substituents on the benzene ring of the indole as well as various electronwithdrawing $N$-substituents could be successfully engaged in the transformation.

Scheme 57. Enantioselective palladium-catalyzed dearomative $(3+2)$ annulation of VCP 240 with 3nitroindoles reported by Shi and co-workers. ${ }^{108}$

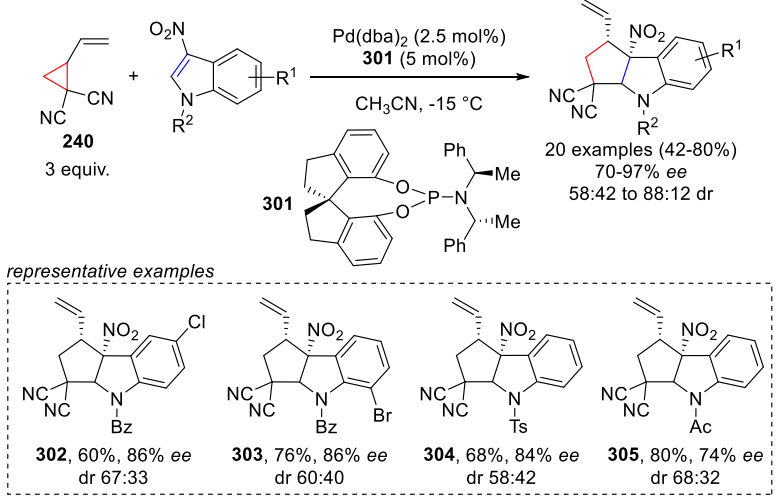

Heteroatom-containing dipolarophiles, such as carbonyl compounds, imines and diazo compounds, could also be used in the palladium catalyzed annulation. Shi and co-workers developed an asymmetric $(3+2)$ annulation between isatins and VCPs, leading to oxindole-fused spirotetrahydrofurans such as 307-314 (Scheme 58). ${ }^{109}$ Employing imidazoline-phosphine ligand 306, they found that addition of lithium chloride as additive improved the diastereoselectivity. Variation of the isatin partner was well tolerated, and VCP substituted with different diester groups could be used in the transformation.
However, substitution on the terminal position of the alkene diminished both the yield and enantioselectivity (product 314).

Scheme 58. Enantioselective palladium-catalyzed $(3+2)$ annulation of VCPs and isatins reported by Shi and coworkers. ${ }^{109}$

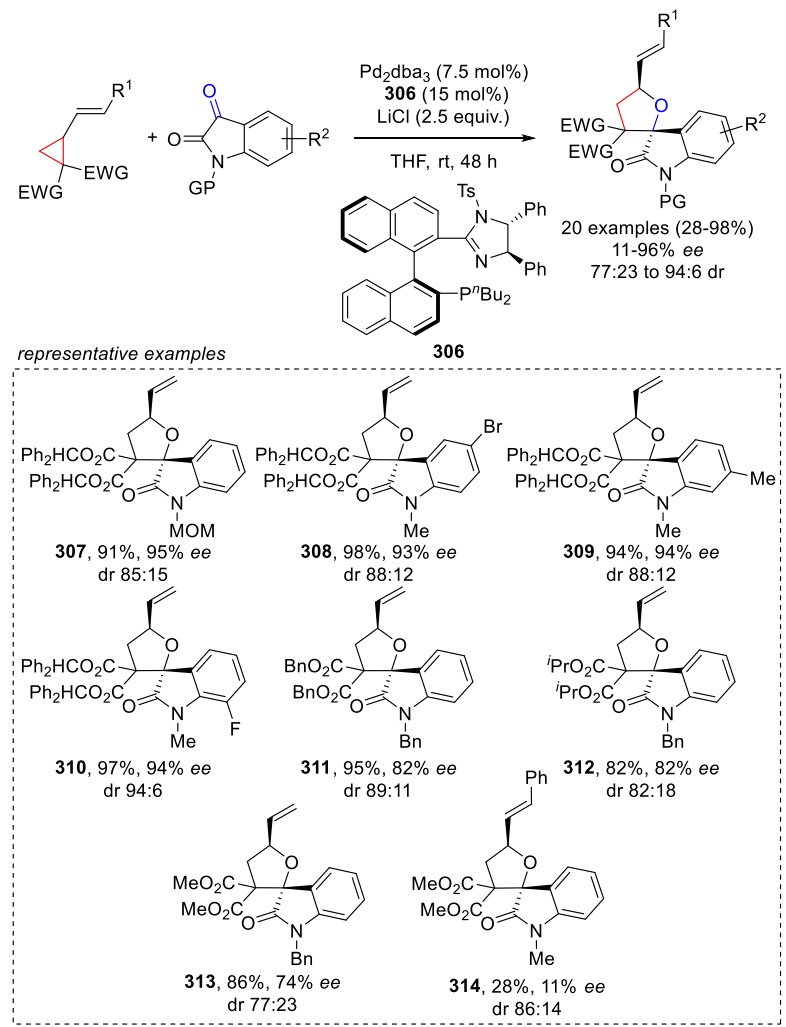

Imines have proven to be more challenging partners in the asymmetric palladium catalyzed $(3+2)$ annulation with VCPs. The first promising results were reported by the groups of $\mathrm{Guo}^{110}$ and Vitale (Scheme 59). ${ }^{111}$ Employing dicyanosubstituted VCP 240 and cyclic sulfonylimine 315, only low levels of enantiocontrol were observed with diphosphine ligands 317 and 318, despite extensive screening.

Scheme 59. First reports of asymmetric induction in the palladium-catalyzed $(3+2)$ annulation of VCP 240 with imine 315 reported by the groups of Guo and Vitale. ${ }^{110,111}$

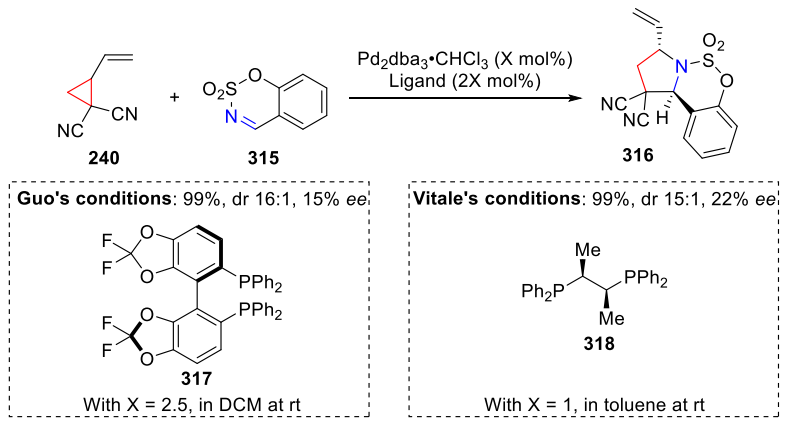

It is only in 2019 that the group of Q. Z. Liu could develop an efficient enantioselective version of the transformation (Scheme 60). ${ }^{112}$ With diester-substituted VCPs combined with phosphoramidite ligand $\mathbf{3 1 9}$ or $\mathbf{3 2 3}$, a range of isatin-derived ketimines as well as different aromatic aldimines delivered the 
corresponding enantioenriched pyrrolidines such as 320-322 or 324-326 in good yields but moderate diastereoselectivities.

Scheme 60. Palladium-catalyzed asymmetric (3+2) annulation of VCPs with imines reported by $Q$. Z. Liu and co-workers. ${ }^{112}$

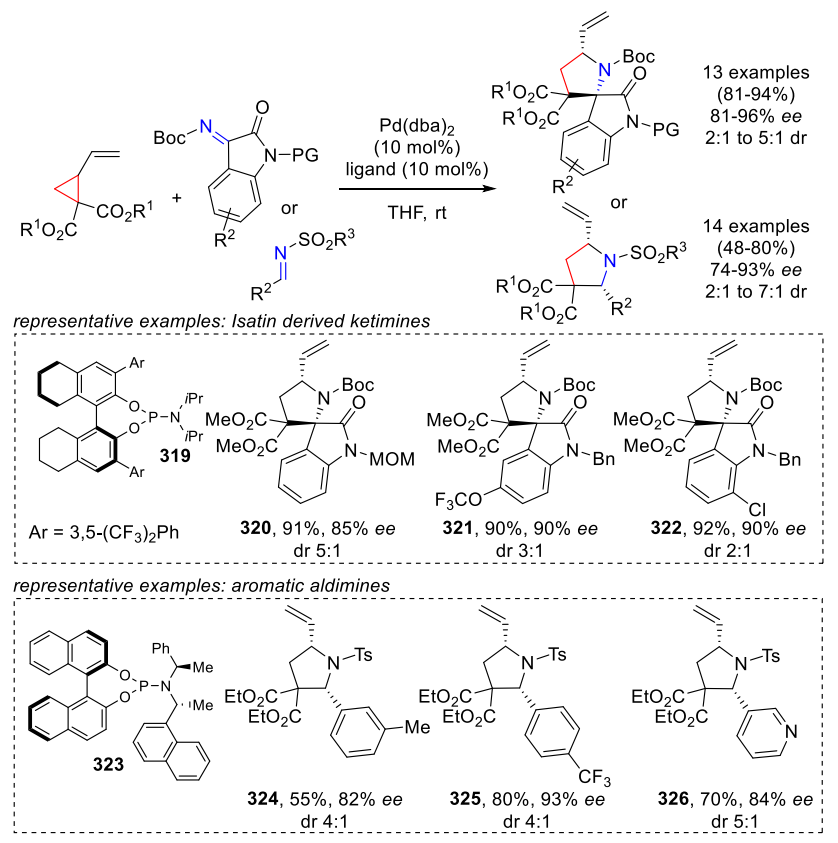

Other five-membered heterocycles could also be accessed using VCPs under palladium catalysis, as illustrated by the work of Shi and co-workers on the asymmetric $(3+2)$ annulation of VCP 256 with 3-diazooxindoles (Scheme 61). ${ }^{113}$ Imidazolinephosphine ligand $\mathbf{3 2 7}$ gave the highest level of enantiocontrol when combined with $\mathrm{Pd}_{2}(\mathrm{dba})_{3} \cdot \mathrm{CHCl}_{3}$ in toluene at $0{ }^{\circ} \mathrm{C}$. Under these conditions, various substituted 3-diazooxindoles were found to be good reaction partners in the annulation, delivering 1,3-indanedione and oxindole-fused spiropyrazolidines such as 328-331 in high yields and good enantioselectivities. X-ray analysis and vibrational circular dichroism spectroscopy of a derivative of $\mathbf{3 2 8}$ allowed the authors to assign the absolute configuration of the spiropyrazolidine derivatives.
Scheme 61. Palladium-catalyzed asymmetric (3+2) annulation of VCP 256 with 3-diazooxindoles reported by Shi and co-workers. ${ }^{113}$

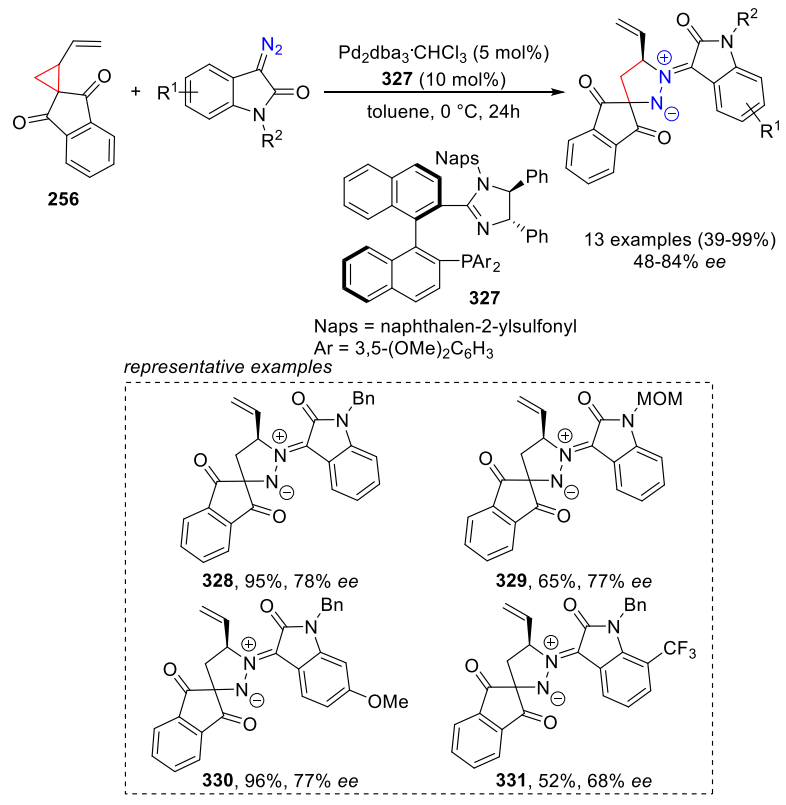

5.1.2. Synergistic palladium-amine catalysis

In 2016, several groups have disclosed a $(3+2)$ annulation for the enantioselective synthesis of polysubstituted cyclopentanes by merging palladium catalysis with iminium/enamine organocatalysis. These methodologies circumvent the use of highly activated dipolarophiles, as well as complex chiral ligands by employing readily available $\alpha, \beta$-unsaturated aldehydes combined with a chiral secondary amine organocatalyst. For this dual activation process, the first report by Vitale and co-workers showed that the combination of $\mathrm{Pd}_{2}(\mathrm{dba})_{3} \cdot \mathrm{CHCl}_{3}$, dppe as ligand and the Hayashi-Jørgensen catalyst 77 led to cyclopentanes such as 332-336 in good yields and high levels of stereocontrol (Scheme 62). ${ }^{114}$ The addition of a Brønsted acid to favor the formation of the in situ generated dipolarophile was crucial to achieve high yields. Various VCPs as well as aromatic enals could be used as partners in the transformation, however aliphatic enals were unsuccessful. The stereochemistry of the major diastereoisomer was proposed to arise from the reaction of intermediate enamine $\mathbf{I}$ in the favored chair conformation to give cyclopentane II. 
Scheme 62. Asymmetric (3+2) annulation of VCPs with enals using synergistic palladium-amine catalysis reported by Vitale and co-workers. ${ }^{114}$

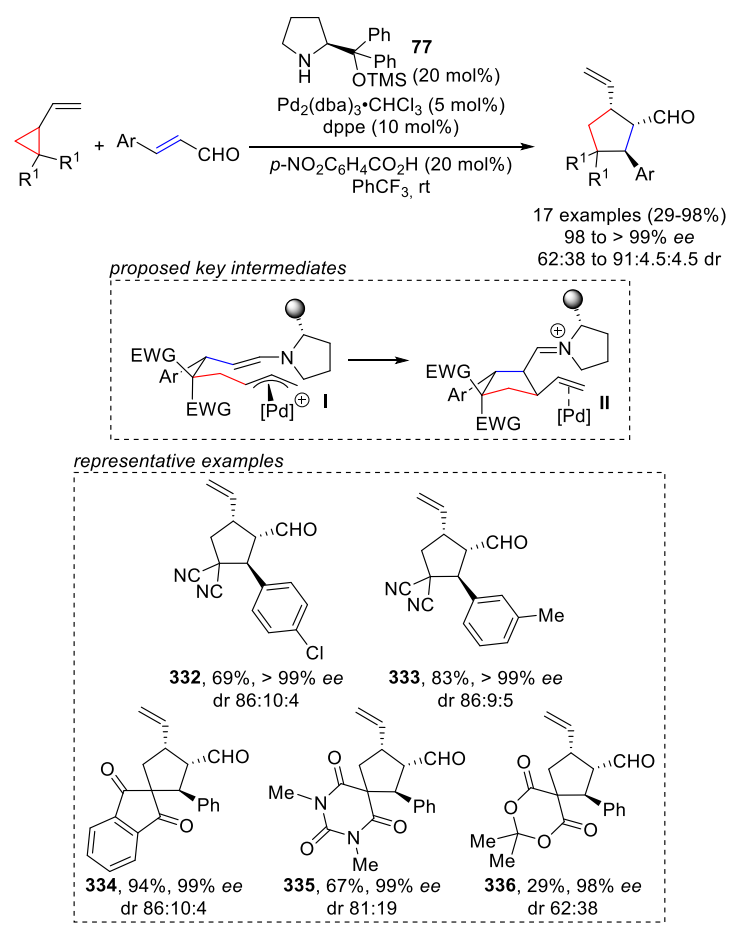

Employing a closely related catalytic system, a similar transformation was reported by Wang and co-workers using diester substituted VCPs. ${ }^{115}$ In these two first examples, the addition of diphosphine ligands led to an improved yield and diastereoselectivity. In contrast, Rios and co-workers showed that employing diketo-substituted VCP $\mathbf{2 5 6}$ did not require any ligand on the palladium to deliver cyclopentanes such as $\mathbf{3 3 7}$ 340 in good yields and enantioselectivities (Scheme 63A). ${ }^{116} \mathrm{At}$ room temperature in ethyl acetate, a variety of aromatic and aliphatic enals could be successfully engaged in the transformation. A year later the same group further expanded this transformation to cyanoesters substituted VCPs, leading to enantioenriched cyclopentanes such as 341-344 bearing four contiguous stereocenters (Scheme 63B). ${ }^{117}$ In 2019, Veselý and co-workers reported the successful extension of this catalytic system to vinylcyclopropane azlactones, providing an efficient access to spirocyclic cyclopentanes such as $\mathbf{3 4 5 - 3 4 8}$ in good yields, high enantioselectivities and moderate diastereoselectivities (Scheme 63C). ${ }^{118}$
Scheme 63. Enantioselective (3+2) annulation of VCPs with enals using synergistic palladium-amine catalysis reported by the groups of Rios and Veselý. ${ }^{116-118}$
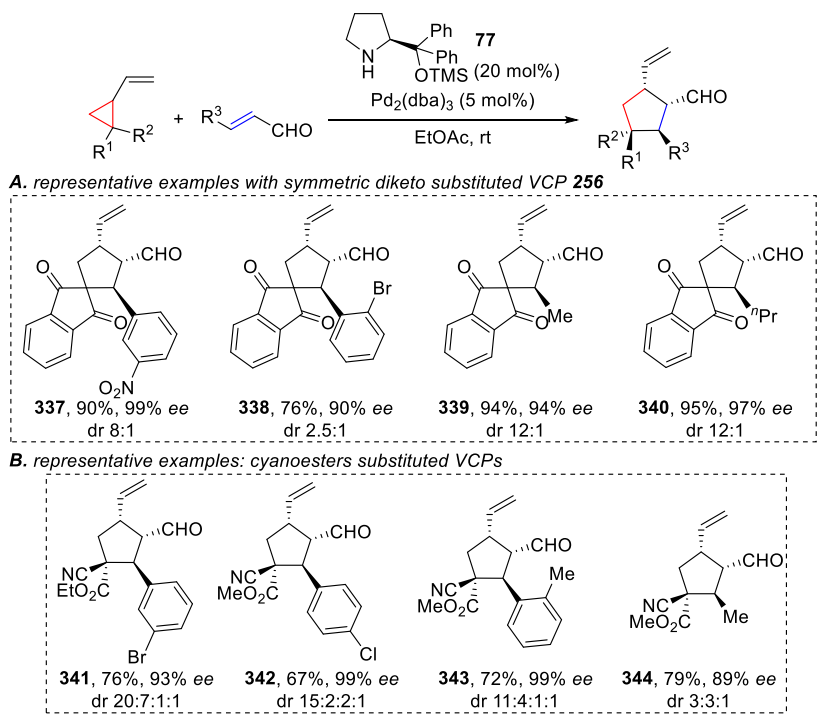

C. representative examples: $V C P$ azlactones

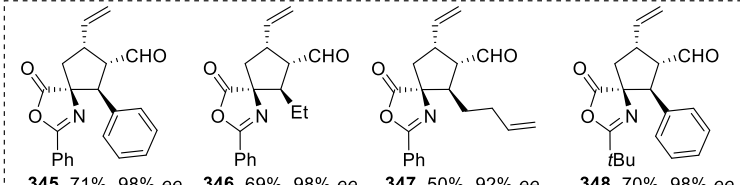

$345,71 \%, 98 \%$ ee $\quad 346,69 \%, 98 \%$ ee $\quad 347,50 \%, 92 \%$ ee $\quad 348,70 \%, 98 \%$ ee dr $4: 1 \quad$ dr $4: 1$ dr 3: dr 2:

Jørgensen and co-workers reported the asymmetric $(3+2)$ annulation between VCPs and $\alpha, \beta$-unsaturated aldehydes using the same strategy (Scheme 64). ${ }^{119}$ Both dicyano- and cyanoester substituted VCPs could be used under the same optimized conditions, delivering cycloadducts such as 349-352 in high enantioselectivities and moderate diastereoselectivities.

Scheme 64. Enantioselective (3+2) annulation of VCPs with enals using synergistic palladium-amine catalysis reported by Jørgensen and co-workers. ${ }^{119}$

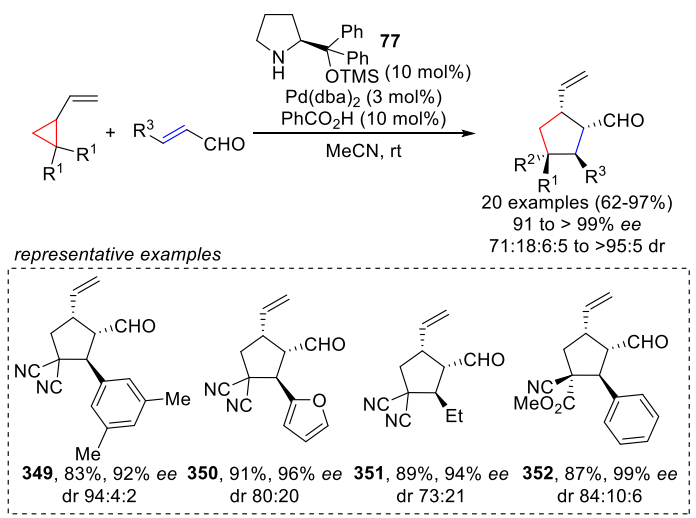

\subsubsection{Rhodium catalysis}

While the use of VCPs as three carbon partners in $(3+2)$ annulations has been extensively studied with palladium, examples involving other transition metals remain scarce. Indeed, a single example of rhodium-catalyzed asymmetric $(3+2)$ annulation involving VCPs was reported by $\mathrm{Yu}$ and coworkers in 2012 (Scheme 65). ${ }^{120}$ In general, (5+2) annulations are favored with rhodium catalysis (See section 5.2). 
Employing 1-yne-VCPs, this process led to various enantioenriched carbo- and heterobicyclo[3.3.0] products such as 354-358 in good yields in the presence of $\left[\mathrm{Rh}(\mathrm{CO})_{2} \mathrm{Cl}\right]_{2}$ and $\mathrm{H}_{8}$-BINAP (353). In contrast to palladium catalysis, on one hand only intramolecular cases were reported, but on the other hand no activating electron-withdrawing groups were needed on the cyclopropane. Bulkier substituents on the alkyne were found to increase the enantioselectivity. DFT studies highlighted that the alkyne insertion step is stereodetermining and governed by steric repulsion between the BINAP backbone and the substituent on the alkyne (transition state I). The authors also disclosed that the desired product could not be observed in the case of longer tethers between the cyclopropyl and the alkyne, and in the case of 1-ene-VCPs.

Scheme 65. Enantioselective rhodium-catalyzed intramolecular (3+2) annulation of 1-yne-VCPs reported by Yu and co-workers. ${ }^{120}$

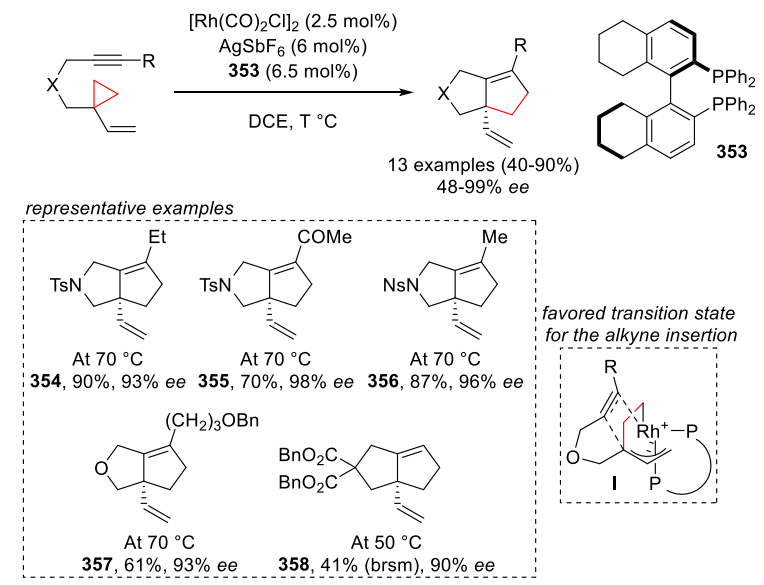

\subsubsection{Thiyl radical catalysis}

In addition to transition metals, radical chemistry was also successfully applied to the development of catalytic asymmetric ring opening reactions of VCPs. Upon radical addition onto the alkene double bond and formation of an alkyl radical next to the three-membered ring, VCPs undergo ring opening to form homoallylic radicals that can be readily engaged in $(3+2)$ annulations. This process was first exploited in the context of asymmetric catalysis by Maruoka and co-workers in 2014, with the use of chiral thiol catalyst 359 (Scheme 66). ${ }^{121}$ The active thiyl radical $I$ is first generated by oxidation and then reacts with the VCP leading to electrophilic radical II. The latter then add to the electron-rich olefin to generate alkyl radical III. In case of $\mathrm{R}^{1}=\mathrm{R}^{2}$, both absolute and relative stereochemistry are set in the second $\mathrm{C}-\mathrm{C}$ bond forming event to form the product. During this step, thiyl radical $\mathbf{I}$ is also released. The transformation was shown to tolerate various VCPs, and delivered functionalized cyclopentanes such as 360-363 in high yields, diastereo- and enantioselectivities. Concerning the alkene partners, the reaction was limited to vinyl ethers and one single example of ene-carbamate that led to a significant decrease in stereoselectivity.
Scheme 66. Thiyl radical catalyzed enantioselective $(3+2)$ annulation of VCPs with electron-rich alkenes reported by Maruoka and co-workers. ${ }^{121}$

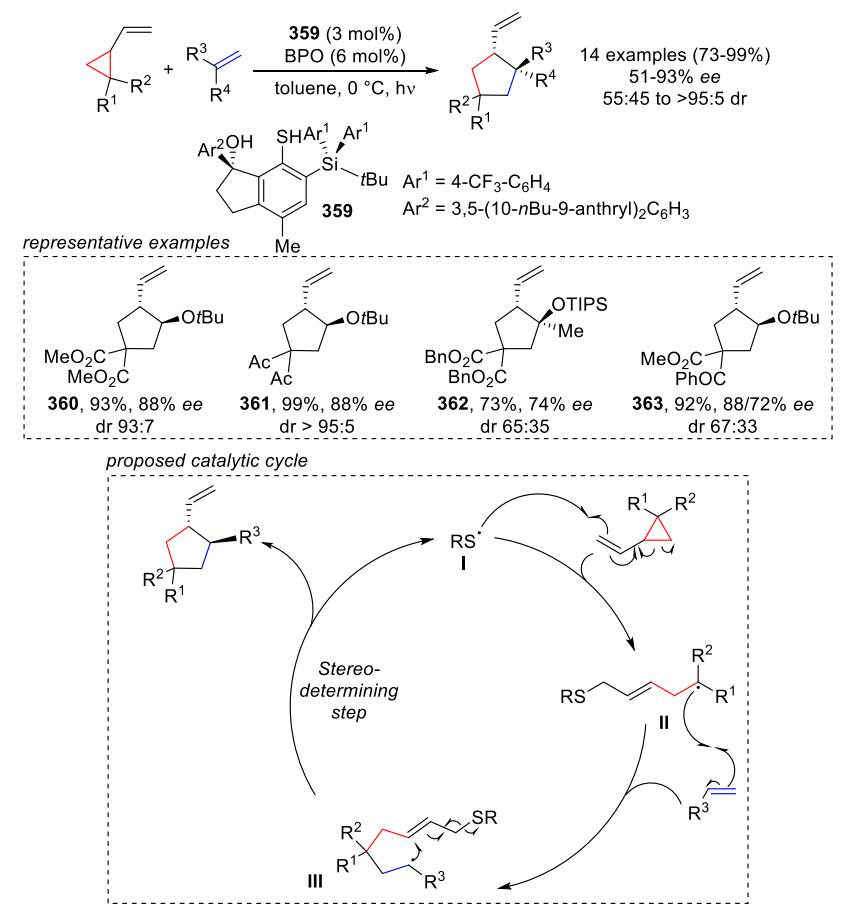

Building upon this work, Miller and co-workers reported the enantioselective $(3+2)$ annulation of VCPs with activated alkenes catalyzed by disulfide-bridged peptide 364 (Scheme 67). ${ }^{122}$ The thiyl radical is generated by homolytic cleavage of the disulfide bond upon exposure to UV light. Fine-tuning of the substitution on the proline of the peptide was key to reach high stereocontrol. Amide-substituted VCPs led to the highest level of enantiocontrol, due to a possible $\mathrm{H}$-bonding interaction with the peptide (intermediate I). Diverse heteroatomsubstituted olefins were suitable partners in the radical mediated annulation to give cyclopentanes such as 365-369. However 1,1-disubstituted alkenes were found to be less reactive and other types of olefins did not deliver the desired products. 
Scheme 67. Disulfide-bridged peptide-catalyzed asymmetric $(3+2)$ annulation of VCPs with activated alkenes reported by Miller and co-workers. ${ }^{122}$

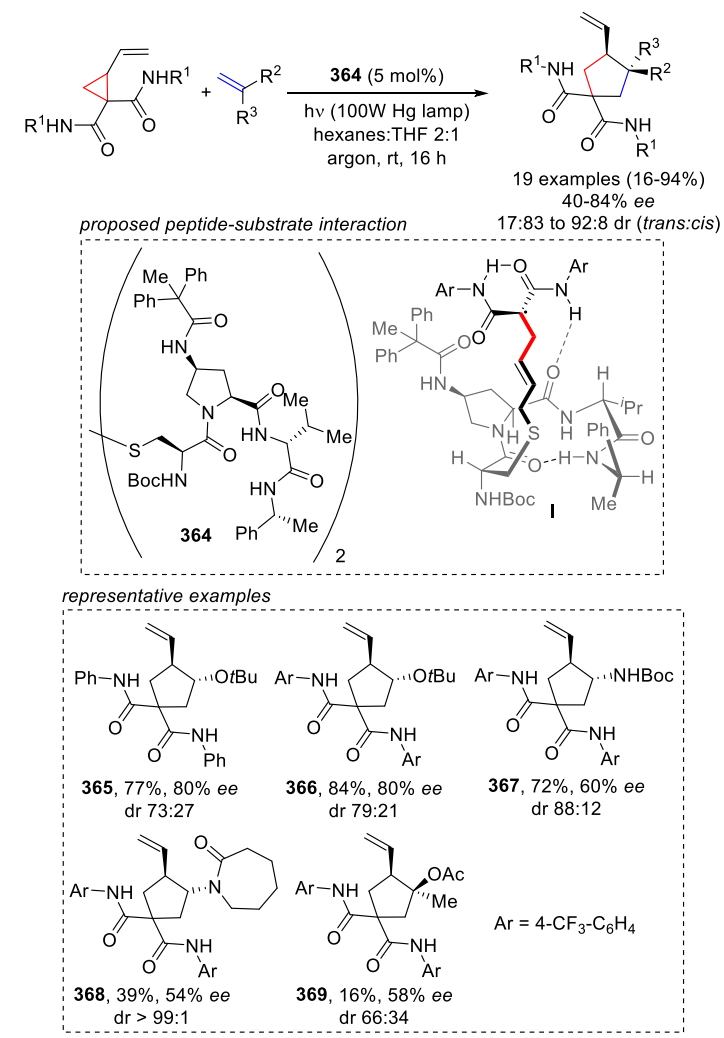

\section{2. $\quad(5+2)$ Annulations}

Besides their extensive use in $(3+2)$ annulations, VCPs were also exploited as five carbons synthons in asymmetric intramolecular processes. The most successful catalyst in this case is rhodium. In contrast to palladium catalysis, no activating electron-withdrawing groups are needed on the cyclopropane.

After studying the racemic rhodium-catalyzed intramolecular $(5+2)$ annulation of VCPs with alkynes and alkenes, ${ }^{123,124}$ Wender and co-workers reported the first enantioselective example in 1998 (Scheme 68). ${ }^{125}$ Starting from VCP 370 bearing a tethered alkene, they could form cycloadduct $\mathbf{3 7 2}$ in $80 \%$ yield and $63 \%$ ee using a combination of $\mathrm{Rh}\left(\mathrm{C}_{2} \mathrm{H}_{4}\right)_{2} \mathrm{Cl}$ and $(S, S)$-Chiraphos (371) as catalyst.

Scheme 68. First example of asymmetric induction in an intramolecular rhodium-catalyzed (5+2) annulation of $\mathrm{VCP}$ 370 reported by Wender and co-workers. ${ }^{125}$

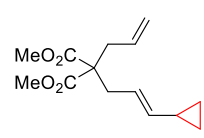

370
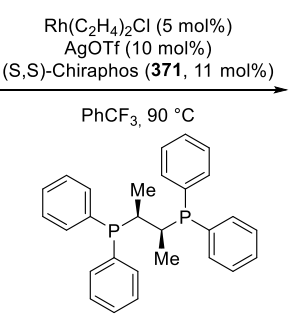

(S,S)-Chiraphos (371)

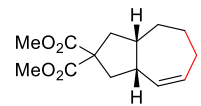

$372,80 \%, 63 \%$ ee
The same group further studied this transformation and reported in 2006 an optimized system using [((R)-BINAP) $\mathrm{Rh} \mathrm{SbF}_{6}$ (373) as catalyst (Scheme 69). ${ }^{126}$ Different substitutions and tethers incorporating nitrogen or malonates were tolerated under the reaction conditions to give annulation products such as 374-376. However, long reaction times and higher temperatures were required for certain substrates. In addition to alkenes, VCPs bearing tethered alkynes also gave bicyclic compounds such as $\mathbf{3 7 7}$, albeit in lower enantioselectivities.

Scheme 69. Asymmetric rhodium-catalyzed intramolecular $(5+2)$ annulation of VCPs reported by Wender and coworkers. ${ }^{126}$

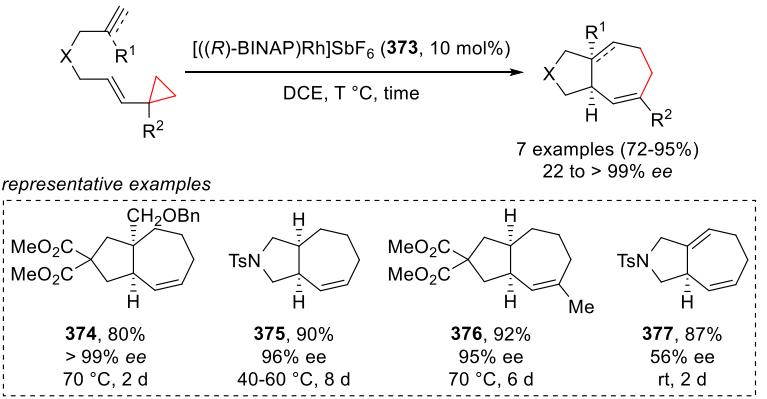

Hayashi and co-workers reported an efficient asymmetric (5+2) annulation for VCPs bearing tethered alkynes (Scheme 70). ${ }^{127}$ Both aryl and alkyl substituents were tolerated on the alkyne, as well as different types of tethers on the VCP substrates under optimized conditions with $\left[\mathrm{Rh}\left(\mathrm{C}_{2} \mathrm{H}_{4}\right)_{2} \mathrm{Cl}\right]_{2}$ as catalyst and phosphoramidite ligand 263 to give cycloadducts such as $\mathbf{3 7 8}$ 382 with high yields and enantioselectivities.

Scheme 70. Rhodium-catalyzed enantioselective intramolecular $(5+2)$ annulation of VCPs reported by Hayashi and co-workers. ${ }^{127}$

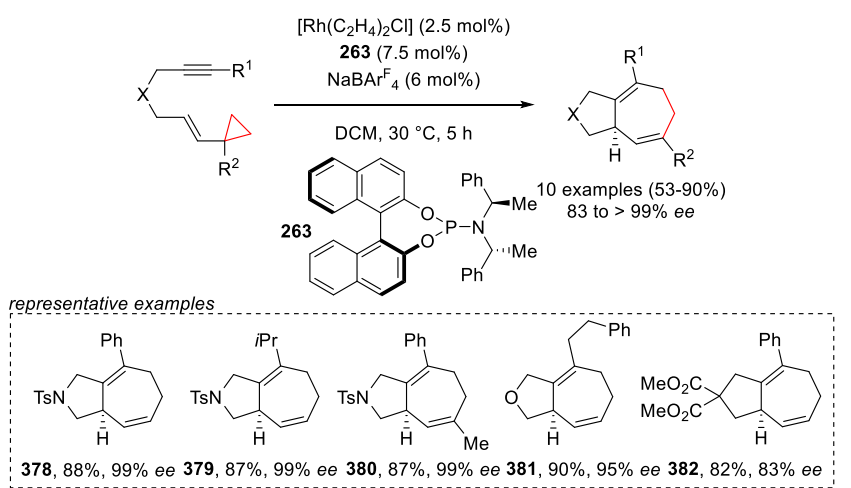

Ynamide tethered VCPs were reported by Anderson and coworkers to be also suitable substrates in intramolecular asymmetric $(5+2)$ annulations (Scheme 71). ${ }^{128}$ DFT calculations showed that the oxidative coupling was the ratelimiting and enantiodetermining step, and $\mathbf{I}$ was found to be the lowest energy transition state for this step. This model, along with experimental observations, helped to optimize the phosphoramidite ligand, with $\mathbf{3 8 3}$ giving the highest level of enantiocontrol with $\left[\mathrm{Rh}\left(\mathrm{C}_{2} \mathrm{H}_{4}\right)_{2} \mathrm{Cl}\right]$ as catalyst. Under these conditions, ynamide tethered VCPs gave [5.3.0] azabicycles such as $\mathbf{3 8 4}$ and $\mathbf{3 8 5}$ in high yields and enantioselectivities. In addition, enantioenriched substrates were engaged in the transformation and furnished products such as $\mathbf{3 8 6}$ or $\mathbf{3 8 7}$ in high diastereoselectivity thanks to a matched substrate-catalyst system. 
Scheme 71. Rhodium-catalyzed enantioselective intramolecular $(5+2)$ annulation of ynamide tethered VCPs reported by Anderson and co-workers. ${ }^{128}$

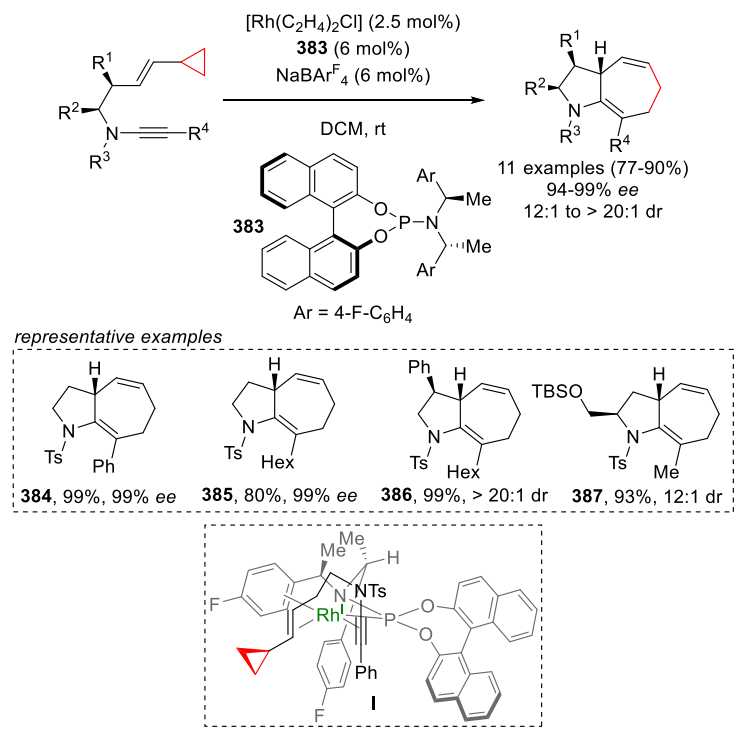

\section{3. (4+3) Annulations}

A palladium catalyzed enantioselective dearomatization of anthranils by (4+3) annulation with VCPs was reported by You and co-workers (Scheme 72). ${ }^{129}$ The addition of triethylborane as a co-activating Lewis acid was crucial to achieve satisfying yields. Using PHOX ligand 388, excellent diastereoselectivities $(16: 1$ to $>20: 1 \mathrm{dr})$ and high enantioselectivies were observed for annulation products such as 389-393. Various substituents on the phenyl rings of the anthranil partner were tolerated, as well as different ester groups on the VCP.

Scheme 72. Palladium-catalyzed enantioselective $(4+3)$ annulation of VCPs with anthranils reported by You and co-workers. ${ }^{129}$

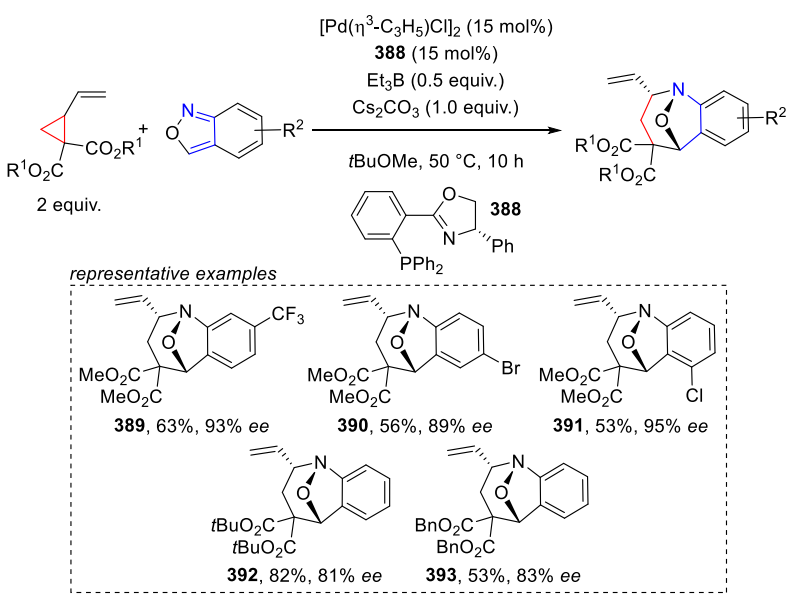

\subsection{Ring expansion}

The activation of the double bond of 1-vinylcyclopropan-1-ols with an electrophile (metal, proton or halogen) leads to a carbocation intermediate, which can undergo a 1,2-WagnerMeerwein alkyl (semi-pinacol) shift to give cyclobutanones. The first catalytic asymmetric Wagner-Meerwein rearrangement was described by Trost and co-workers through the formation of a $\pi$-allyl complex, enabling the enantioselective ring expansion of vinylcyclopropanols (Scheme 73). ${ }^{130}$ Differentiation of the two prochiral faces of the alkene was successfully achieved by the formation of a $\pi$ allylpalladium complex in the presence of chiral ligand 227. Cyclobutanones such as 394-396 were formed in good yields and enantioselectivities. The same chemistry was applied to vinylcyclobutanols producing enantioenriched cyclopentanone products.

Scheme 73. Catalytic asymmetric Wagner-Meerwein rearrangement of vinylcyclopropanols reported by Trost and co-workers. ${ }^{130}$

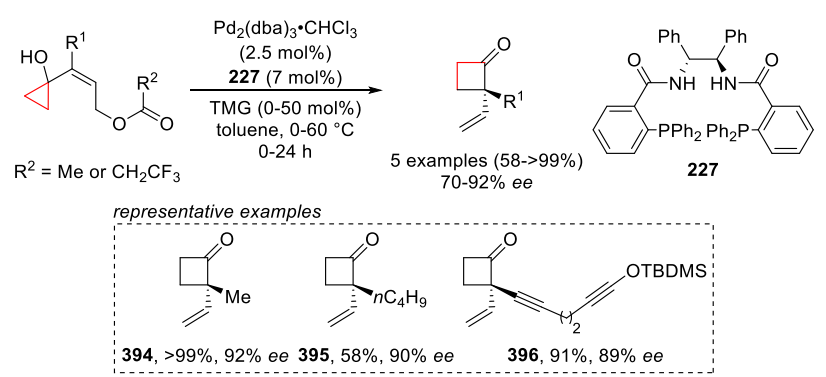

Another method for the enantioselective synthesis of cyclobutanones was described by Toste and Kleinbeck through the ring expansion of 1-allenylcyclopropanols catalyzed by a chiral phosphine gold(I) complex 397 (Scheme 74). ${ }^{131}$ This ring expansion was promoted by the coordination of a gold(I) catalyst to the internal double bond of the allene leading to a Wagner-Meerwein shift. Optimization of the reaction conditions revealed that sterically hindered substituents on the phosphine as well as low temperatures were required to reach high $e e$ values. A series of cyclobutanones bearing a vinylsubstituted quaternary stereocenter such as 398-401 were obtained in high yields and high enantioselectivities.

Scheme 74. Gold-catalysed asymmetric ring expansion reaction of 1-allenylcyclopropanols reported by Toste and Kleinbeck. ${ }^{131}$

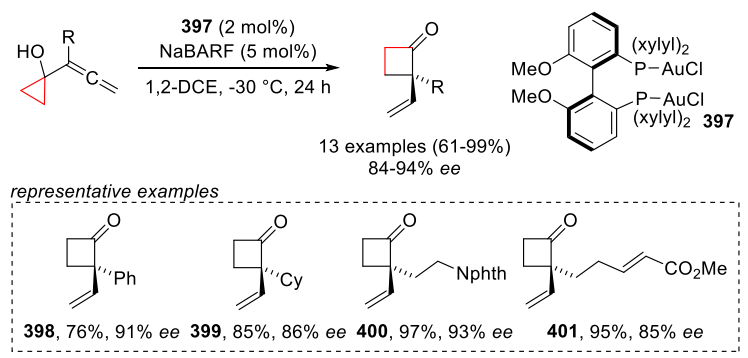

The enantioselective Wagner-Meerwein rearrangement of vinylcyclopropanols was also studied by Alexakis and coworkers. Their approach relied on anionic phase transfer catalysis (PTC): the interaction of a halonium ion with a chiral anion promotes an enantioselective halogenative WagnerMeerwein rearrangement. The fluorination of vinylcyclopropanols with Selectfluor (402) was first studied using phosphates as chiral anions (Scheme 75). ${ }^{132}$ In a $1: 1$ mixture of fluorobenzene and $n$-hexane containing the highly hindered phosphoric acid $\mathbf{4 0 3}$ and $\mathrm{Na}_{3} \mathrm{PO}_{4}$ as a base, $\beta$-fluoro spirolactones such as 404-407 were obtained in good yields with excellent enantioselectivities and diastereoselectivities. This enantioselective fluorination/ring expansion cascade was also applied to cyclobutanols to give cyclopentanones. Without 
an aromatic substituent on the allylic alcohol, ß-fluoro spirolactones were obtained with only moderate stereoselectivities.

Scheme 75. Organocatalytic fluorination-induced WagnerMeerwein rearrangement of vinylcyclopropanols reported by Alexakis and co-workers. ${ }^{132}$

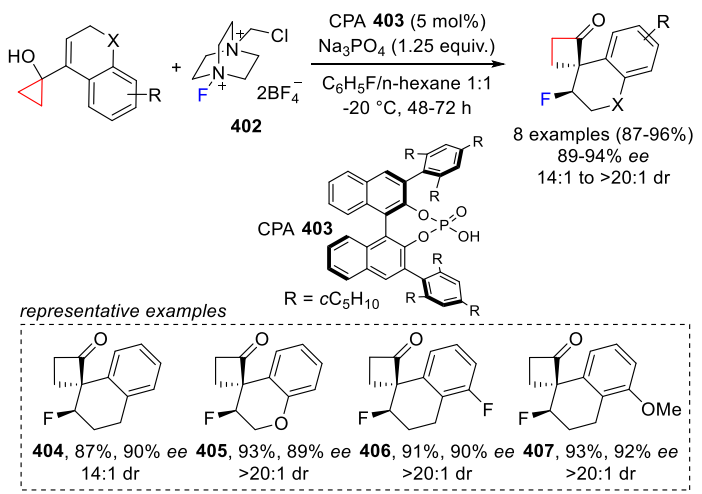

Brominative and iodinative semi-pinacol rearrangements, as well as stereodivergent halogenations were developed by the same authors using reagents 408 and 409 together with CPA (Chiral Phosphoric Acid) catalyst 410 (Figure 8). ${ }^{133,134}$ These halogenative phase-transfer methodologies were also discussed in a comprehensive study including scope, limitations and mechanism. ${ }^{135}$
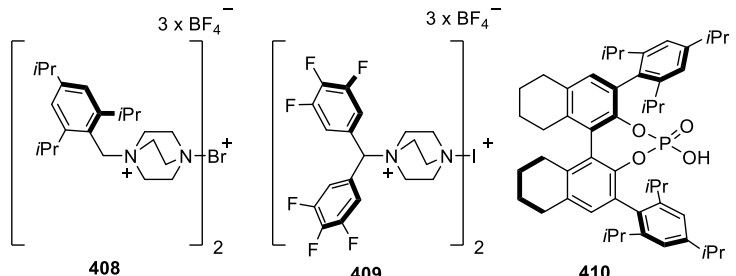

Figure 8. Asymmetric PTC systems developed by Alexakis and co-workers. ${ }^{133}$

An asymmetric gold catalyzed rearrangement of cyclopropyl1,6-enynes was reported by Voituriez and co-workers (Scheme 76). ${ }^{136}$ The transformation proceeded efficiently in the presence of the digold precatalyst $\mathbf{4 1 1}$ in wet toluene $\left(35 \mathrm{~mol} \% \mathrm{H}_{2} \mathrm{O}\right)$, leading to enantioenriched cyclobutanone derivatives such as 412-416. The reaction tolerated different tethers, as well as substitution on the alkyne, alkene and cyclopropyl groups, leading to the rearranged products in good yields and enantioselectivities, but moderate diastereoselectivities.
Scheme 76. Gold-catalyzed asymmetric rearrangement of enyne-substituted VCPs reported by Voituriez and coworkers. $^{136}$

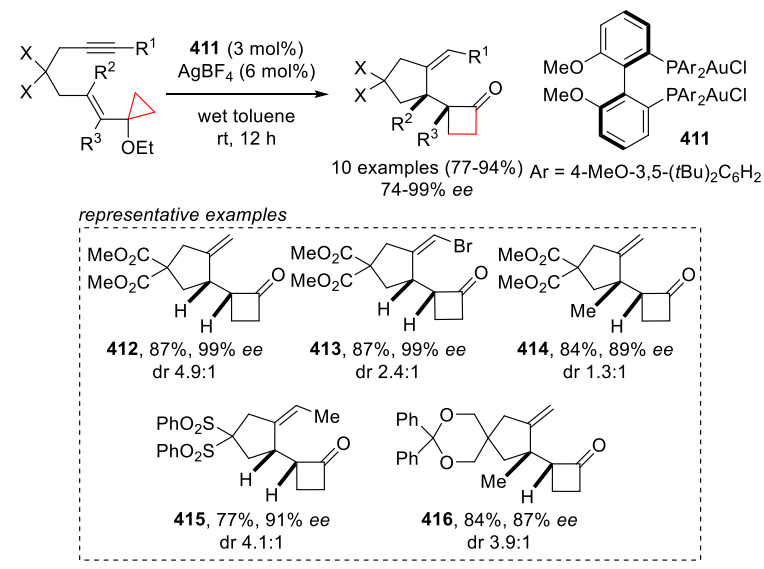

\subsection{Ring-opening reactions}

In comparison to annulation and ring expansion processes, only scattered examples of enantioselective ring-opening reactions have appeared. Trost and co-workers reported a palladiumcatalyzed asymmetric allylic alkylation of indole derivatives with VCPs (Scheme 77). ${ }^{137}$ In this transformation the $\pi$-allyl palladium complex generated from the ring opening of the VCP serves as an electrophile. The addition of triethylborane leads to an increased acidity of the indole $\mathrm{NH}$ bond upon complexation, inducing protonation of the malonate anion of the zwitterionic $\pi$-allyl palladium complex, which becomes only electrophilic. The enantioselectivity of the transformation was ensured by employing ligand $\mathbf{2 2 7}$ already found optimal by the Trost group in the context of the asymmetric $(3+2)$ annulation with VCPs. In addition to the allylic alkylation of 3 -substituted $1 H$-indoles to give dearomatized products such as $\mathbf{4 1 7}$, a tandem cyclization could be achieved in the same conditions with indoles possessing a tethered nucleophile, leading to highly functionalized tricyclic scaffolds such as 418-420.

Scheme 77. Palladium-catalyzed enantioselective nucleophilic ring opening of VCPs with indoles reported by Trost and co-workers. ${ }^{137}$

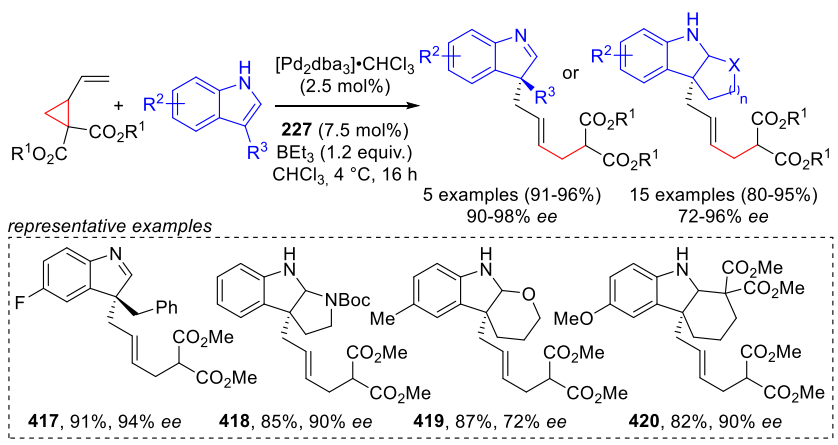

Inspired by the precedents in the field of asymmetric iridiumcatalyzed allylation of carbonyls, ${ }^{138,139}$ the groups of Krische and Johnson conjointly reported the electrophilic functionalization of VCPs with carbonyl compounds (Scheme 78). ${ }^{140}$ When diester-substituted VCP 231 was exposed to cyclometalated iridium catalyst 421, a nucleophilic $\pi$ allyliridium complex $\mathbf{I}$ is formed, enabling a reductive coupling 
with aldehydes or alcohols. This unprecedented Umpolung of VCPs to give a 1,3-bisnucleophilic synthons led to open adducts such as $\mathbf{4 2 2 - 4 2 4}$ in high yields and enantioselectivities in the presence of alcohols. Aldehydes could also be used as coupling partners, with the addition of 2 equivalents of isopropanol as terminal reductant, delivering the desired products in comparable yields and enantioselectivities, but higher diastereoselectivities. When submitted to Krapcho decarboxylative conditions, the products could be converted into the corresponding cis-4,5-disubstituted $\delta$-lactones.

Scheme 78. Iridium-catalyzed asymmetric electrophilic ring-opening of VCP 231 with aldehydes and alcohols reported by the groups of Johnson and Krische. ${ }^{140}$

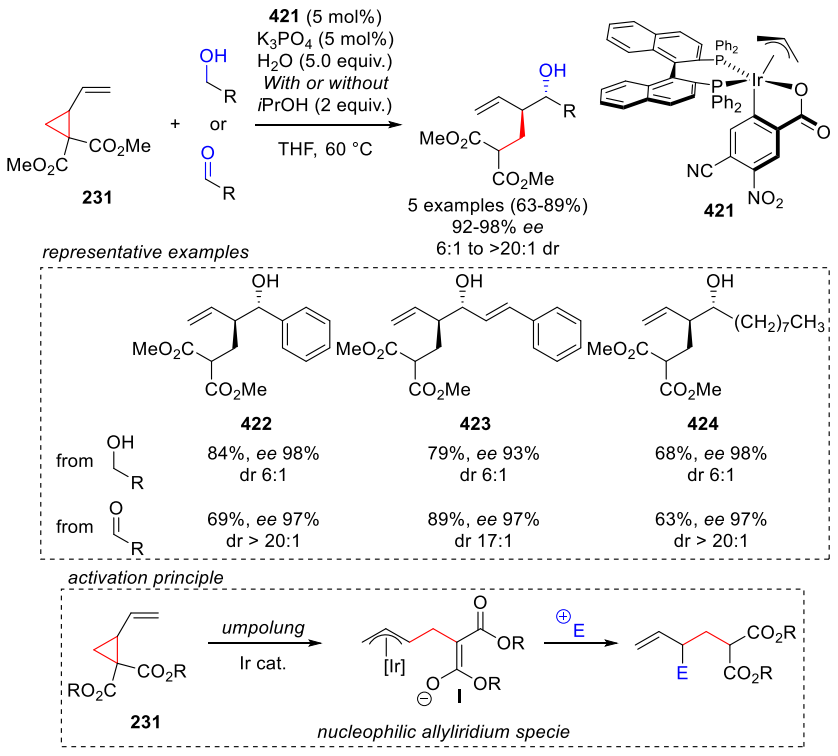

Finally, an asymmetric 1,5-difunctionalization of donorsubstituted cyclopropanes could be achieved with VCPs based on a radical mechanism: Wang and co-workers reported an enantioselective copper-catalyzed 1,5cyanotrifluoromethylation of VCPs (Scheme 79). ${ }^{141}$ Using a radical relay strategy, a combination of $\mathrm{Cu}(\mathrm{acac})_{2}$ with $\mathrm{BOX}$ ligand 426 along with a slight excess of Togni-II reagent $\mathbf{( 4 2 5 )}$ and TMSCN gave access in high yields and stereocontrol to 1,5difunctionalized products such as 427-430. The homoallylic radical formed after ring opening of the VCP is trapped by the copper catalyst, which then delivers the cyanotrifluoromethylated product upon reductive elimination under ligand control.
Scheme 79. Copper-catalyzed enantioselective 1,5cyanotrifluoromethylation of VCPs reported by Wang and co-workers. ${ }^{141}$

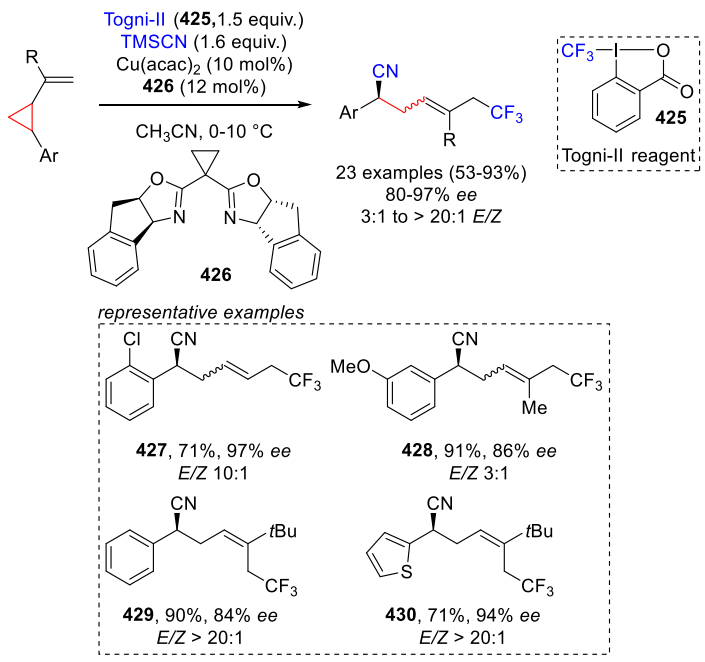

\section{Alkylidenecyclopropanes}

Bearing a strained exocyclic double bond, alkylidenecyclopropanes (ACPs) are an important class of three membered carbocycles for the development of enantioselective ring-opening reactions. Especially, ACPs are privileged substrates for transition metal-mediated transformations. A well-studied mode of activation is via the formation of a metallacyclobutane by oxidative addition, which then reacts in annulations with diverse partners. Another important process involves the formation of a cyclopropylmetal species, that easily undergoes a $\beta$-carbon elimination to form a homoallylic metal complex. The latter can then lead to difunctionalized linear products. Asymmetric ring expansions were also reported, proceeding through 1,2-rearrangements upon in situ activation of the exo-methylene group.

\subsection{Annulation reactions}

Only limited examples of enantioselective transition metalcatalyzed annulations with ACPs have been developed so far. Mechanistically, the reactions occur via a metallacyclobutane intermediate formed after coordination of the double bond to a metal complex, followed by migratory insertion/oxidative addition into a $\mathrm{C}-\mathrm{C}$ bond of the three-membered carbocycle.

The first efforts towards an enantioselective palladiumcatalyzed (4+3) intramolecular annulation of ACPs and dienes were reported in 2007 by Mascareñas and co-workers (Scheme 80). ${ }^{142}$ The presence of a conjugate diene in substrate 431 was expected to favor the formation of the seven-membered cycloadduct $\mathbf{4 3 3}$ over product $\mathbf{4 3 4}$ coming from $(3+2)$ annulation. Oxidative cyclometallation with an alkene and the ACP first give palladium complex I. A $\pi$-allyl rearrangement leads then to intermediate II, followed by reductive elimination to give the seven-membered ring product. Direct reductive elimination from I leads to the five-membered ring product. The seven-membered cycloadduct was obtained as the major product in an encouraging ee value of $64 \%$ with ligand $\mathbf{4 3 2}$. 
Scheme 80. Enantioselective palladium-catalyzed $(4+3)$ intramolecular annulation of ACPs and dienes reported by Mascareñas and co-workers. ${ }^{142}$
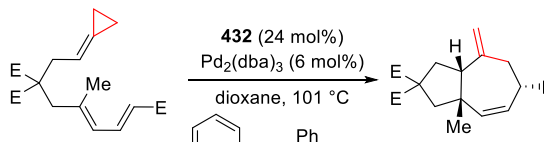

431, $\mathrm{E}=\mathrm{CO}_{2} \mathrm{Et}$<smiles>C1=CCCC1</smiles><smiles>C[C@H](c1ccccc1)N([C@H](C)c1ccccc1)p1oc2ccccc2c2ccccc2o1</smiles>$$
432
$$

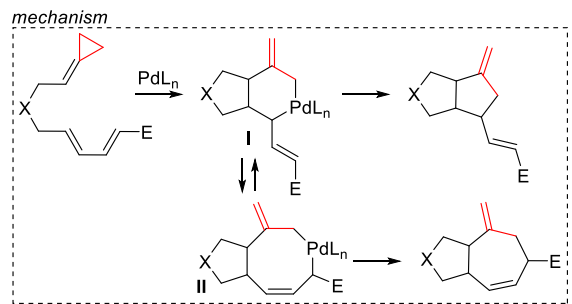

A second proof of concept for an enantioselective transitionmetal-catalysed annulation with ACPs was achieved by Evans and co-workers in 2012 (Scheme 81). ${ }^{143}$ In this report, a $[3+2+1]$ carbocyclization of ACP $\mathbf{4 3 5}$ with $\mathrm{CO}$ was performed in the presence of chiral bidentate $P, N$-ligand 436 and $\mathrm{Rh}(\mathrm{COD})_{2} \mathrm{OTf}$ providing cis-fused bicyclohexenone $\mathbf{4 3 7}$ in $75 \%$ yield with $89 \%$ enantiomeric excess.

Scheme 81. Rh-catalysed [3+2+1] carbocyclization of ACPs with $\mathrm{CO}$ reported by Evans and co-workers. ${ }^{143}$
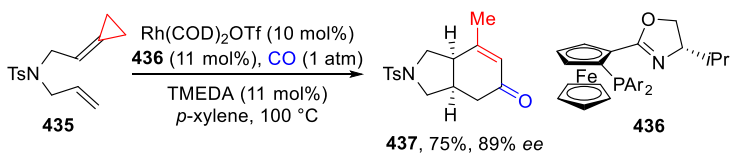

Building upon their preliminary results, Mascareñas and coworkers reported in 2018 an efficient palladium-catalyzed enantioselective $(3+2)$ intramolecular annulation of ACPs with alkenes (Scheme 82A). ${ }^{144}$ The highly hindered Vapol-derived phosphoramidite $\mathbf{4 3 8}$ was found to be key to achieve high level of enantioinduction. Combined with $\mathrm{CpPd}(\pi$-cinnamyl) as catalyst, a series of $c i$-fused 5,5-bicyclic adducts such as 439441 could be obtained in good yields and enantioselectivities ranging from 44 to $94 \%$ and complete diastereoselectivity in most cases. When tethered 1,3-dienes were used instead of alkenes, the less bulky phosphoramidite ligand $\mathbf{2 6 3}$ could be used to promote the corresponding asymmetric $(4+3)$ annulation (Scheme 82B). 5,7-Bicyclic systems such as $\mathbf{4 4 2}$ or 443 bearing up to three stereocenters could be obtained in good yields and $e e$ values ranging from 54 to $94 \%$. Regioselectivity issues were however encountered and the $(3+2)$ adduct was also obtained in some cases.
Scheme 82. Enantioselective $(3+2)$ and $(4+3)$ intramolecular annulations of ACPs with alkenes and dienes reported by Mascareñas and co-workers. ${ }^{144}$

A.
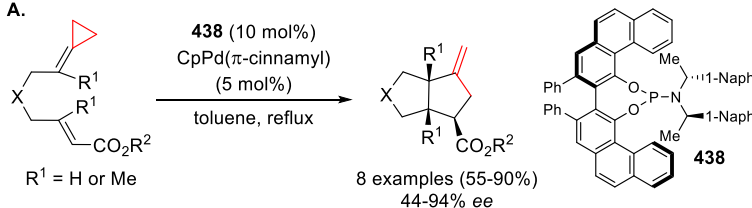
8 examples $(55-90 \%)$
$44-94 \%$ ee

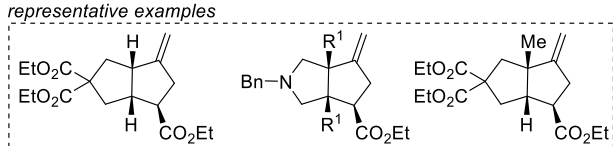

B. $\mathbf{4 3 9}, 89 \%, 94 \%$ ee $\quad \mathbf{4 4 0}, 70 \%, 84 \%$ ee $\quad \mathbf{4 4 1}, 85 \%, 92 \%$ ee
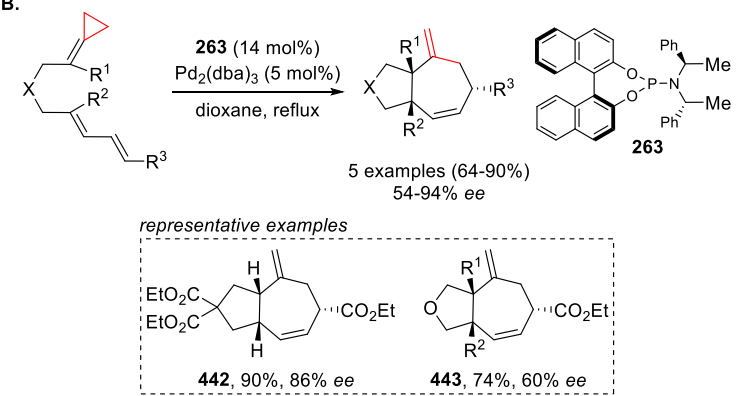

The groups of Lautens reported an enantioselective ringopening/cyclization cascade of ACPs with aldimines catalyzed by a chiral magnesium complex (Scheme 83). ${ }^{145}$ Magnesium activates the cyclopropane for nucleophilic attack by iodide through coordination to the amide group to give enolate $\mathbf{I}$. $\alpha$ Alkylation of I with aldimines followed by cyclization affords methylene pyrolidines. An enantioselective variant of this reaction was performed using BOX ligand $\mathbf{4 2 6}$ in a substoichiometric amount. High loading $(45 \mathrm{~mol} \%)$ was required to avoid the racemic background reaction. A series of transmethylenepyrrolidine derivatives such as $\mathbf{4 4 4 - 4 4 7}$ were formed in good yields and $e e$ values ranging from 47 to $86 \%$.

Scheme 83. Enantioselective magnesium-catalysed ringopening/cyclization cascade of ACPs with aldimines reported by Lautens and co-workers. ${ }^{145}$
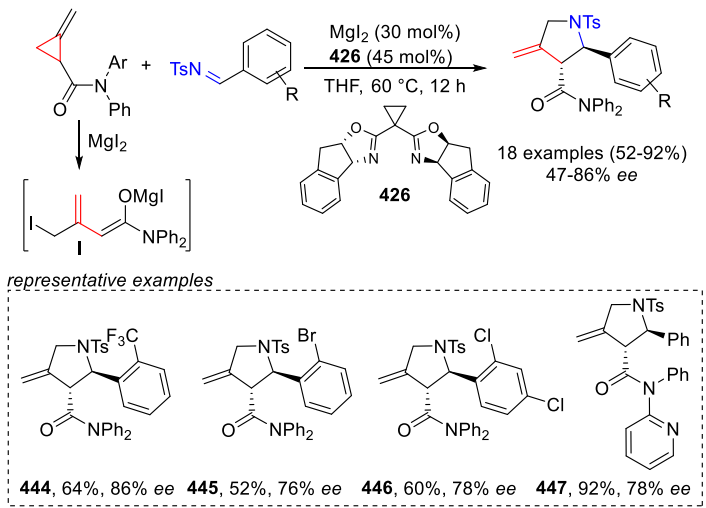

\subsection{Ring expansions}

Ring expansion reactions of ACPs take advantage of the reactive nature of the exo-methylene bond, by forming in situ a reactive intermediate that can trigger a 1,2 rearrangement. Fukumoto and co-workers reported already in 1992 a tandem Katsuki-Sharpless asymmetric epoxidation of 2,2-disubstituted 
ACPs followed by an enantiospecific ring expansion through a 1,2 migration (Scheme 84). ${ }^{146}$ Depending on the choice of the tartrate used in the asymmetric epoxidation, both enantiomers of the chiral cyclobutanones products could be accessed. Alkyland aryl-substituted cyclopropylidene alcohols delivered cyclobutanones such as 448-450 in good yields and high enantioselectivities. The same group applied this strategy to the enantioselective total synthesis of various biologically active compounds. ${ }^{147-152}$

Scheme 84. Tandem asymmetric epoxidation / ring expansion of 2,2-disubstituted cyclopropylidene alcohols reported by Fukumoto and co-workers. ${ }^{146}$

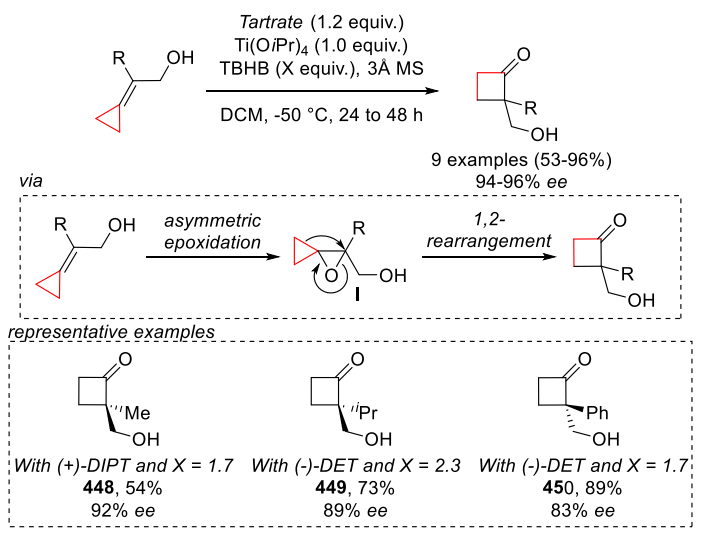

Shi and co-workers reported a cascade asymmetric epoxidation, ring expansion and Baeyer-Villiger oxidation of ACPs to access enantioenriched $\gamma$-aryl- $\gamma$-butyrolactones such as $\mathbf{4 5 2 - 4 5 4}$ (Scheme 85). ${ }^{153}$ Employing a combination of glucose-derived oxazolidinone 451 and an excess of oxone, the transformation tolerated a variety of tri- and tetrasubstituted benzylidenecyclopropanes.

Scheme 85. Cascade asymmetric epoxidation / ring expansion / Baeyer-Villiger oxidation of ACPs reported by Shi and co-workers. ${ }^{153}$
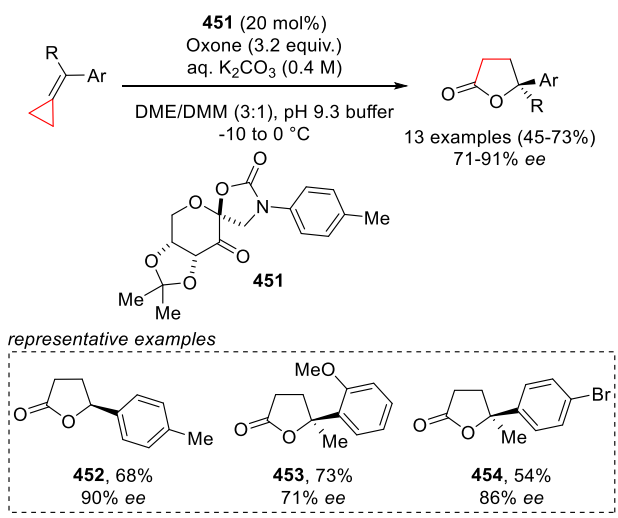

Gagné and co-workers reported an asymmetric gold-catalyzed ring expansion of alkyne tethered ACPs through cycloisomerization, providing an efficient access to bicyclo[4.2.0] octadienes (Scheme 86). ${ }^{154}$ Using $(R, R)-i \mathrm{Pr}-$ $\operatorname{DuPHOS}(\mathrm{AuCl})_{2}(\mathbf{4 5 5})$ as catalyst in nitromethane at $0{ }^{\circ} \mathrm{C}$, several substituted cycloadducts such as $\mathbf{4 5 6 - 4 5 9}$ could be obtained in good yields, with ee values ranging from 28 to $70 \%$. The reaction was proposed to proceed via alkyne activation by the gold catalyst followed by 6-endo-dig cyclization to give carbocation I. Wagner-Meerwein shift then gives ringexpanded intermediate II, which can be drawn as a goldcarbene resonance structure III. Finally, a 1,2-hydride shift leads to the observed diene product.

Scheme 86. Gold-catalyzed asymmetric cycloisomerization of alkyne tethered ACPs reported by Gagné and coworkers. ${ }^{154}$

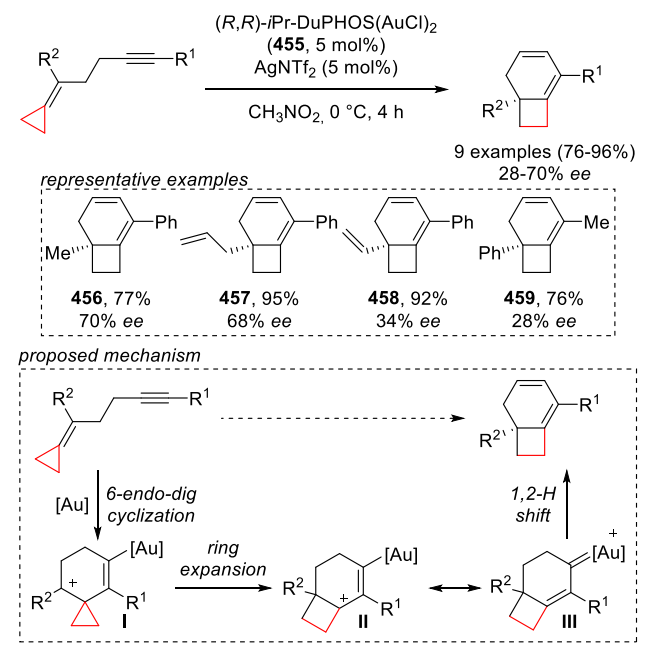

Building upon the work of Gagné and co-workers, the group of $\mathrm{Yu}$ reported the cycloisomerization of alkyne-tethered ACPs, containing one extra heteroatom in the tether to access 7membered rings (Scheme 87). ${ }^{155}$ Using $\mathbf{4 6 0}\left(\mathrm{AuSbF}_{6}\right)_{2}$ as optimal catalyst, this process led to a variety of enantioenriched azepine-fused cyclobutanes such as $\mathbf{4 6 1}$ and $\mathbf{4 6 2}$ in high yields and enantioselectivities. The reaction could also be used for accessing oxygen-based heterocycles such as $\mathbf{4 6 3}$ in moderate yields and enantioselectivities.

Scheme 87. Gold-catalyzed asymmetric cycloisomerization of yne-ACPs reported by $\mathrm{Yu}$ and co-workers. ${ }^{155}$
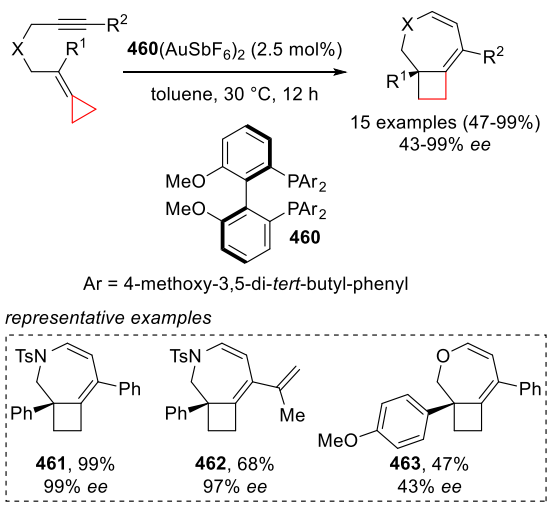

A one-pot enantioselective cyclopropanation of meso-ACP 464 followed by a thermally induced rearrangement to access piperidine-fused trans-cycloalkenes such as $\mathbf{4 6 6 - 4 6 8}$ was reported by Murakami and co-workers (Scheme 88). ${ }^{156}$ In this transformation the asymmetric cyclopropanation is catalyzed by $\mathrm{Rh}_{2}[(S)-\mathrm{NTTL}]_{4}(\mathrm{NTTL}=N$-naphthoyl-tert-leucinate $)(\mathbf{4 6 5})$ and the required rhodium carbene intermediate is generated from a $N$-sulfonyl-1,2,3-triazole. The obtained $\alpha$-imino spiropentane is then heated at $120{ }^{\circ} \mathrm{C}$ under microwave irradiation to trigger a ring-opening rearrangement leading to 
the enantioenriched bicyclic product (transition state I). Different substituted triazoles were compatible with the reaction conditions. Moreover, the authors demonstrated that it was possible to start directly from terminal alkynes, by simply adding mesyl azide and $10 \mathrm{~mol} \%$ of CuTC in the first step, without erosion of the enantioselectivity. Other bicyclic mesoACPs of different ring sizes were also investigated. With ninemembered bicyclic substrate $\mathbf{4 6 9}$ and triazole $\mathbf{4 7 0}$, the rearrangement proceeded at $40{ }^{\circ} \mathrm{C}$ without microwave irradiation and furnished the corresponding trans-cyclodecene 471 in $93 \%$ yield and $97 \%$ ee. However, using a sevenmembered bicyclic ACP led to a significant decrease in yield along with a mixture of isomers.

Scheme 88. One-pot enantioselective cyclopropanation of meso-ACPs / rearrangement for the synthesis of transcycloalkenes reported by Murakami and co-workers. ${ }^{156}$

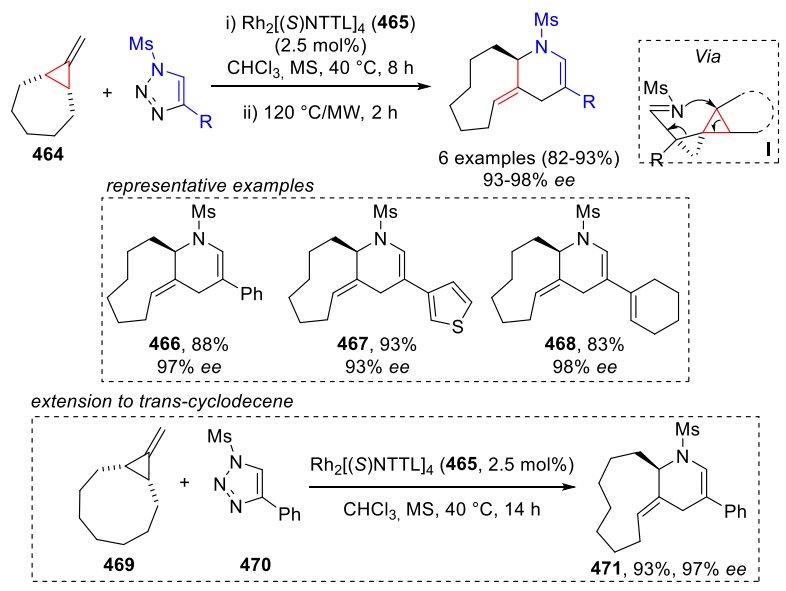

\subsection{Ring-opening / difunctionalization}

Upon regioselective migratory insertion of an appropriately substituted palladium complex onto the double bond of ACPs, a $\beta$-carbon elimination furnishes a homoallylic intermediate, which is able to deliver a difunctionalized open product after reductive elimination. In 2007, Suginome and co-workers reported a palladium-catalyzed asymmetric desymmetrization of meso-ACPs by silaboration (Scheme 89). ${ }^{157}$ They found that a combination of $\mathrm{Pd}(\mathrm{dba})_{2}$ and monophosphine ligand 473 in a 1:1.2 ratio efficiently promoted the challenging proximal C-C bond cleavage of ACPs. In addition, the use of $\mathrm{MePh}_{2} \mathrm{SiB}$ (pin) (472) was key to induce high levels of enantiocontrol. Under these conditions, different bicyclic meso-ACPs afforded desymmetrized products such as $\mathbf{4 7 4}$ or $\mathbf{4 7 5}$ in good yields and enantioselectivities, in varying reaction times (48 to 99 hours). When a non-bicyclic meso-ACP was used, a slight decrease in ee was observed for product $\mathbf{4 7 6}$.
Scheme 89. Palladium-catalyzed enantioselective silaborative $\mathrm{C}-\mathrm{C}$ cleavage of meso-ACPs reported by Suginome and co-workers. ${ }^{157}$

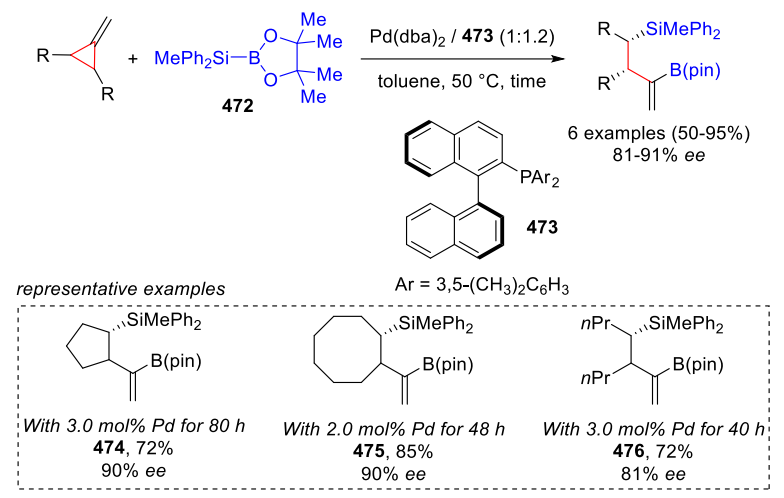

Two years later the same group reported an extension of this methodology to racemic 1-alkyl-2-ACPs, via a kinetic resolution under palladium catalysis (Scheme 90). ${ }^{158}$ This asymmetric silaborative $\mathrm{C}-\mathrm{C}$ cleavage proceeded efficiently again in the presence of $\mathrm{MePh}_{2} \mathrm{SiB}$ (pin) (472), $\mathrm{Pd}(\mathrm{dba})_{2}$ and this time phosphoramidite ligand 263. However complete regioselectivity could not be achieved, as the discrimination between the two proximal C-C bonds turned out to be challenging, with a preference for breaking the less sterically hindered one. The ratio between the two regioisomers $\mathbf{A}$ and $\mathbf{B}$ could be increased up to $86: 14$, by using 3.0 equivalents of the racemic ACP. Alkyl chains of varying length and containing different functional groups were tolerated on the substrate, delivering products such as 477-479 in high yields and enantioselectivities.

Scheme 90. Palladium-catalyzed kinetic resolution of racemic 1-alkyl-2-ACPs via silaborative C-C cleavage reported by Suginome and co-workers. ${ }^{158}$

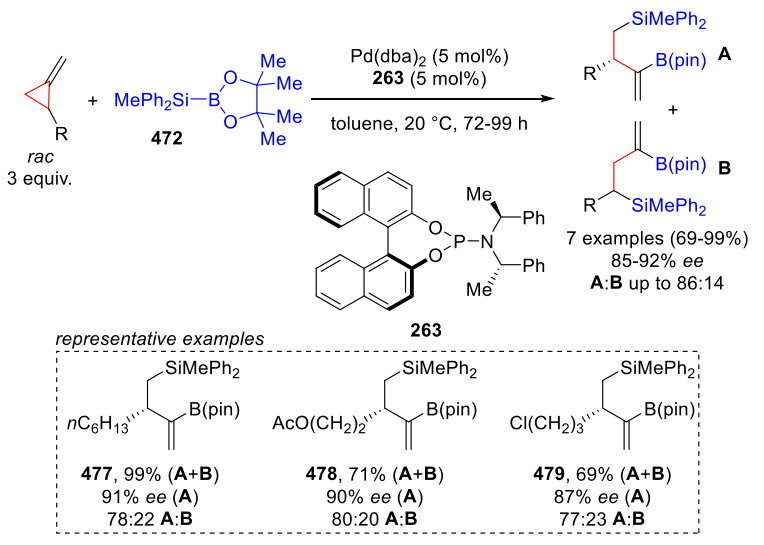

Suginome and co-workers could also apply their polymer-based chiral phosphine ligand $\mathrm{PQXPhos} \mathbf{4 8 0}$ to the palladium catalyzed desymmetrization of meso-ACPs by an asymmetric silaborative cleavage (Scheme 91). ${ }^{159}$ PQXPhos ligand 480 was shown to be superior to classical non-polymeric phosphine ligands in the case of monocyclic and oxygen containing ACPs to give products such as 481-483. In addition, a rate enhancement of the reaction was observed with the polymer based ligands, allowing the transformation to be performed at room temperature or at lower catalyst loading. 
Scheme 91. Palladium-catalyzed desymmetrization of mesoACPs by silaborative cleavage with PQXPho ligand 480 reported by Suginome and co-workers. ${ }^{159}$
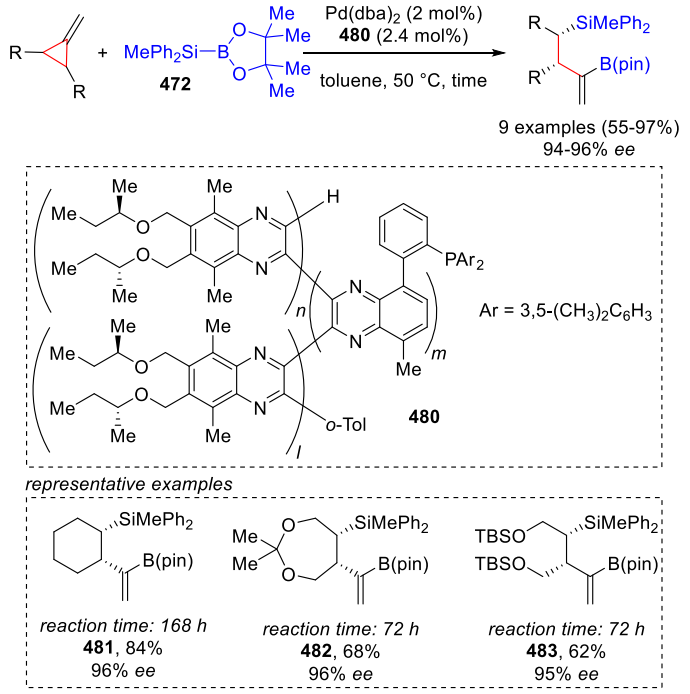

This palladium catalyzed asymmetric silaborative ring cleavage of meso-ACPs was further utilized by Suginome and coworkers as a benchmark reaction to highlight the efficiency of macromolecular helical catalysts, bearing (diarylphosphino)phenyl groups on their side chains. ${ }^{160,161}$

A desymmetrization of meso-ACPs was also reported by Ukaji and co-workers through a palladium-catalysed asymmetric ring-opening/bis(alkoxycarbonylation) reaction (Scheme 92). ${ }^{162}$ According to the authors, this carbonylation proceeds via the carbopalladation of a $\mathrm{Pd}-\mathrm{CO}_{2} \mathrm{Me}$ species followed by ring cleavage of the cyclopropane ring. Asymmetric induction with chiral bisoxazoline ligands was however disappointing, with BOX ligand 484 leading to $\alpha$-methyleneglutarate product in low enantiopurity.

Scheme 92. Palladium-catalyzed asymmetric ringopening/bis(alkoxycarbonylation) reaction through desymmetrization of meso-ACPs reported by Ukaji and coworkers. ${ }^{162}$

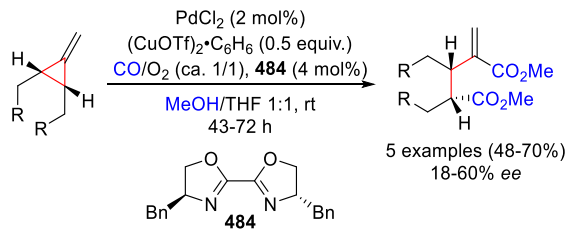

\section{Vinylidenecyclopropanes}

Vinylidenecyclopropanes (VDCPs) are a class of three membered ring carbocycles bearing an allene group, resulting in a high strain energy $\left(51 \mathrm{kcal} \mathrm{mol}^{-1}\right){ }^{35}$ With appropriate substituents, VDCPs are stable and were shown to be suitable substrates for enantioselective ring-opening transformations. ${ }^{36}$ Only a few examples of asymmetric catalysis have been reported so far by the group of Shi, including $\mathrm{C}\left(\mathrm{sp}^{3}\right)-\mathrm{H}$ functionalizations, hydrofunctionalizations, cycloisomerizations and annulations.

An early example of an enantioselective ring-opening process of VDCPs was described by Shi and co-workers involving a gold-catalyzed cycloisomerization of aromatic ring-tethered ene-vinylidenecyclopropanes via a gold carbene intermediate (Scheme 93). ${ }^{163}$ Activation of the VDCP via gold allene complex I leads to the formation of cyclopropyl cation II. Wagner-Meerwein shift then gives gold carbene III, from which the final product is formed through an intramolecular cyclopropanation (IV), leading to polycyclic products such as 486 and 487. Xyl-BINAP (485) was shown to give the highest enantiocontrol (80-87\%). Only four examples of VDCPs were examined in this asymmetric version.

Scheme 93. Gold-catalyzed cycloisomerization of aromatic ring-tethered VDCPs reported by Shi and co-workers. ${ }^{163}$

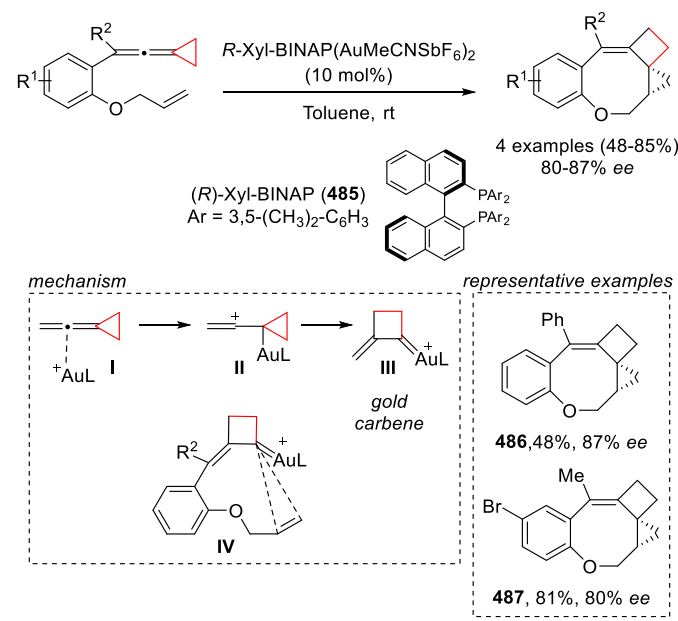

In 2016, the same group developed a $\mathrm{C}\left(\mathrm{sp}^{3}\right)-\mathrm{H}$ functionalization via a gold carbene generated from vinylidenecyclopropanes leading to benzoxepine motifs (Scheme 94). ${ }^{164} \mathrm{C}-\mathrm{H}$ bond insertion (IV) was achieved after fine tuning of the electronic properties of the benzyl ring. The 4-trifluoromethyl group at the para-position was optimal to get the right reactivity of the benzylic $\mathrm{C}-\mathrm{H}$ bond towards $\mathrm{C}-\mathrm{H}$ functionalization. The combination of chiral bisphosphine ligand $\mathbf{4 6 0}$ with $\left[\mathrm{Au}(\mathrm{MeCN}) \mathrm{SbF}_{6}\right]_{2}$ gave the best enantioselectivities. These conditions were applied to five VDCPs leading to the benzoxepine derivatives such as $\mathbf{4 8 8 - 4 9 0}$ in good yields and good enantiopurities. 
Scheme 94. Asymmetric C(sp $\left.{ }^{3}\right)-\mathrm{H}$ functionalization by a gold carbene generated from VDCPs reported by Shi and co-workers. ${ }^{164}$
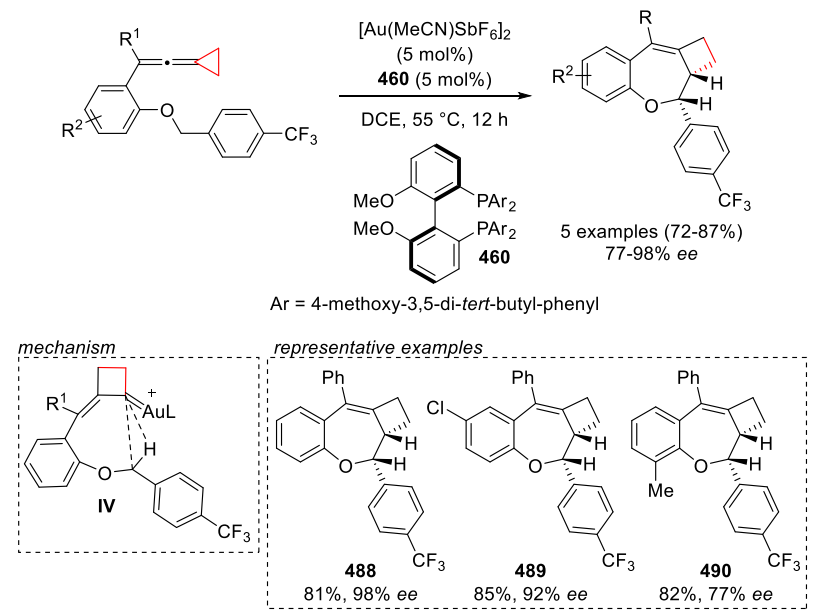

The same group reported a cycloisomerization/cross coupling of keto-VDCPs with alkynes proceeding through rhodium/silver synergistic catalysis and enabling the regioselective and enantioselective formation of tetrahydropyridin-3-ol tethered 1,4-enynes (Scheme 95). ${ }^{165}$ Both metals are needed to activate separately the VDCP and the alkyne. The proposed mechanism starts by an oxidative addition into the weaker distal C-C bond of VDCP 492 leading to a rhoda-cyclobutene intermediate $\mathbf{I}$ after isomerization. Formation of the oxa-rhodacyclic intermediate II is then obtained through a carbometallation of the ketone. Transmetallation of the Ag alkynyl intermediate III, obtained in situ from terminal alkyne $\mathbf{4 9 3}$, to rhodium gives intermediate IV, which after reductive elimination and protonolysis affords the cycloisomerization/cross coupling product 494 via $\mathbf{V}$. Various chiral bisphosphine ligands were screened to develop an enantioselective transformation. Among biaryl and nonbiaryl bisphosphines, 491 gave the best enantioselectivities while yields were improved with $\mathrm{AgNTf}_{2}$ as silver salt. This asymmetric cycloisomerization/cross coupling reaction was exemplified with numerous keto-VDCPs and alkynes furnishing functionalized six-membered ring systems such as 494-499 with excellent yields and very high degree of enantiopurity. The process is compatible with electronwithdrawing and -donating substituents on the benzene ring of keto-VDCPs as well as aryl- or alkyl-substituted terminal alkynes.
Scheme 95. Rhodium/silver synergistic catalysis enabling cycloisomerization/cross coupling of keto-VDCPs with alkynes reported by Shi and co-workers. ${ }^{165}$

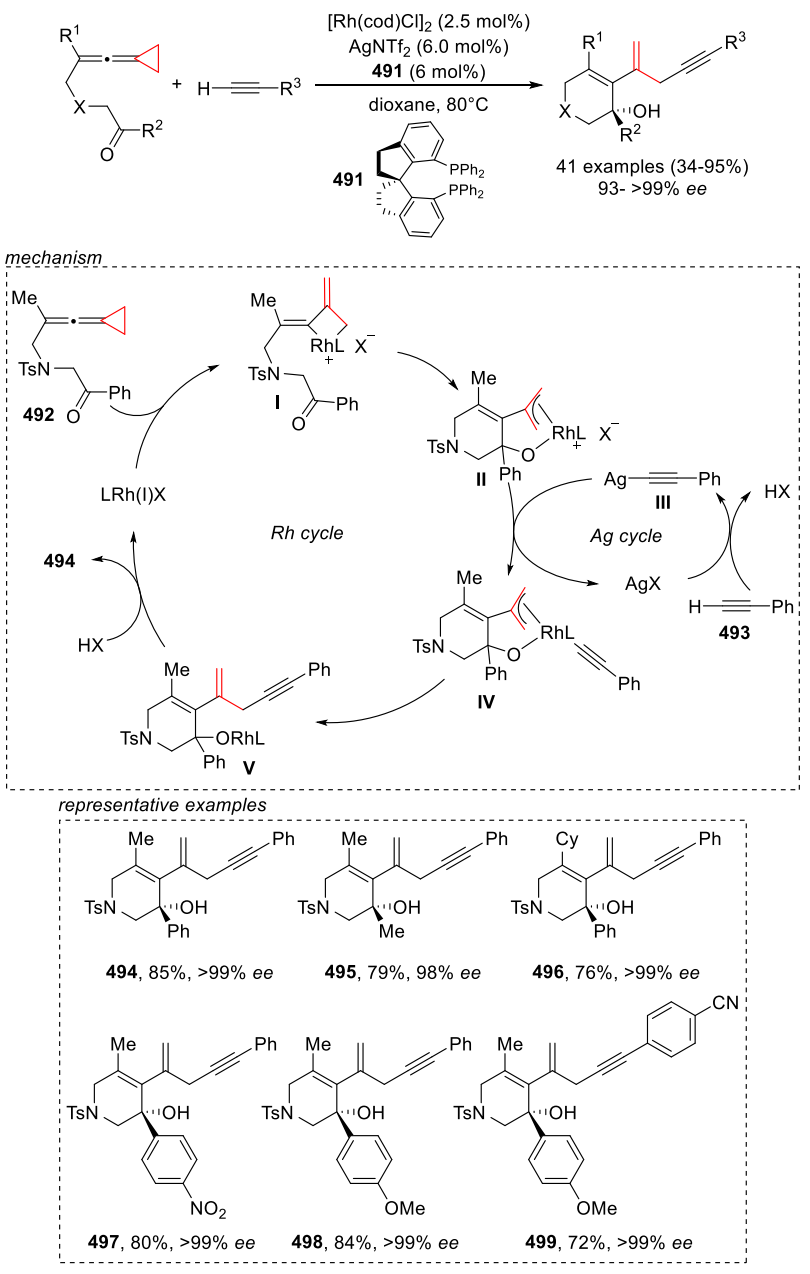

Shi and co-workers also reported an enantioselective hydroamination and hydroindolation of keto-VDCPs under rhodium catalysis following the same mechanism, with an amine or an indole replacing the alkyne nucleophile (Scheme 96). ${ }^{166}[\mathrm{Rh}(\operatorname{cod}) 491] \mathrm{BArF}$ was found to be the best catalyst for the transformation, leading to high yields and enantioselectivities for allyl amine products such as 500-502. The reaction tolerated a range of substituted keto-VDCPs and secondary amines. Indoles could also be used as nucleophiles, under reoptimized conditions, leading selectively to the $\mathrm{C} 3$ functionalized regioisomer. 
Scheme 96. Rhodium-catalyzed enantioselective hydroamination and hydroindolation of keto-VDCPs reported by Shi and co-workers. ${ }^{166}$

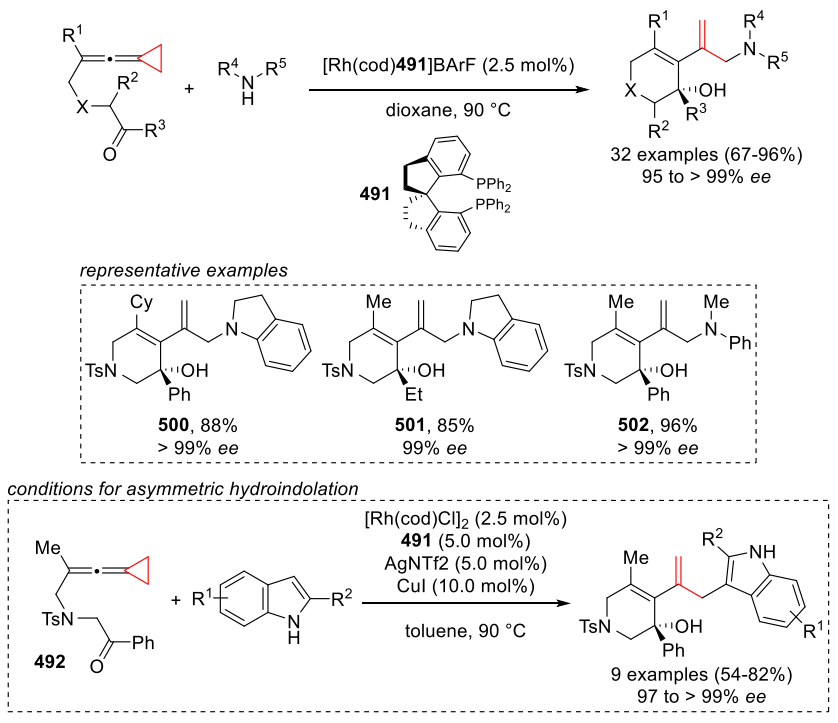

Finally, an asymmetric intramolecular rhodium-catalyzed annulation of ene-VDCPs was also described by the same group (Scheme 97). ${ }^{167}$ Two products could be selectively obtained in this methodology from the same substrate. With $\left[\mathrm{Rh}(\operatorname{cod})_{2}\right] \mathrm{BF}_{4}$ as catalyst, when $(R)$-Tol-BINAP $(\mathbf{5 0 3})$ was used at $80{ }^{\circ} \mathrm{C}$, the corresponding bicycle bearing an exocyclic double bond was obtained as the major product. However, with the same catalyst at $120{ }^{\circ} \mathrm{C}$ with $(R)$-Xyl-BINAP (485) as chiral ligand, the product with an endocyclic double bond was obtained selectively. Under these conditions both type of products (such as 504-506 and 507) were obtained in good yields and high enantioselectivities from diverse substituted ene-VDCPs.

Scheme 97. Rhodium-catalyzed asymmetric intramolecular annulation of ene-VDCPs reported by Shi and coworkers. ${ }^{167}$

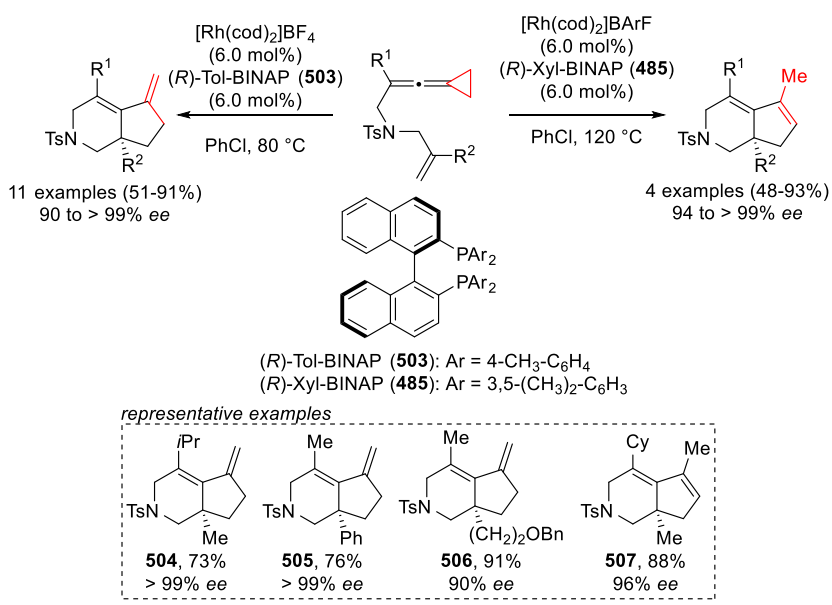

\section{Conclusion and Outlook}

Only a decade ago, the field of enantioselective ring-opening reactions of cyclopropanes was in its infancy. Asymmetric transformations of Donor-Acceptor cyclopropanes were an emerging field, and only isolated examples with other types of cyclopropanes had been reported. At the end of 2019, the situation has changed completely, with more than 100 examples of enantioselective transformations disclosed.

The field of Donor-Acceptor cyclopropanes has truly blossomed, thanks to the availability of new fine-tuned chiral ligands, such as bisoxazolines and $\mathrm{N}$-oxides, or new modes of activation based on amine or carbene catalysis. $(3+2)$ annulations still dominate, but a broad range of other annulations and ring-opening processes have been also developed. Less reactive donor- or acceptor-only cyclopropanes, which had been long considered as not suitable substrates for asymmetric transformations, have now also been successfully used. This has become possible thanks to new activation methods based on transition metal, photoredox or iminium/enamine catalysis.

The use of cyclopropanes bearing unsaturations has also been the focus of intensive research in asymmetric synthesis in the last decade. This field is dominated by the palladium-catalyzed $(3+2)$ annulation of vinylcyclopropanes (VCPs), but other types of annulations, ring expansions and ring-opening reactions have also started to appear using palladium or other transition metal catalysts. Among those, the rhodium-catalyzed $(5+2)$ annulation has been especially successful. Innovative approaches involving carbocation or radical intermediates have further enriched the chemistry of VCPs. The more strained systems with exo double-bonds present in alkylidene- and vinylidene- cyclopropanes (ACPs and VDCPs) have now also been exploited in ring-opening reactions, although the field is less mature. New examples of annulations, ring expansions and difunctionalization reactions are nevertheless promising for future developments.

In summary, enantioselective ring-opening reactions of cyclopropanes have developed tremendously over the last two decades. They have become now valuable tools to access enantioenriched building blocks for synthetic and medicinal chemistry. There is still ample room for further developments, especially using recently introduced modes of activation, such as photoredox, transition-metal or organo-catalysis.

\section{AUTHOR INFORMATION}

Corresponding Author

*E-mail: jerome.waser@epfl.ch

\section{Author Contributions}

†These authors contributed equally.

\section{ORCID}

J. Waser: 0000-0002-4570-914X

\section{Notes}

The authors declare no competing financial interest.

\section{Biographies}


Vincent Pirenne studied chemistry at the University of Namur in Belgium, where he first obtained his Master's degree with Honors in 2014. He then moved to Bordeaux (France) for his doctoral studies in the group of Prof. Yannick Landais (at the University of Bordeaux) and graduated in 2018. His research focused on the development of tin-free radical methodologies for the total synthesis of an alkaloid natural product. Currently, Vincent is in the group of Prof. Jérôme Waser at the Ecole Polytechnique Fédérale de Lausanne (EPFL) as a postdoctoral fellow where his research focuses on ring-opening reactions of D-A cyclopropanes.

Bastian Muriel studied chemistry at the University of Bordeaux in France. He conducted his master thesis in the group of Prof. Jérôme Waser at EPFL, working on the palladium-catalyzed difunctionalization of olefins. He obtained his master degree with Honors in 2016 at the University of Bordeaux, and then returned to Lausanne, where he is currently working towards his Ph.D. His research interests focus on the development of new radical-based methodologies exploiting ring strain.
Jérôme Waser was born in Sierre, Valais, Switzerland. He studied chemistry at ETH Zurich, where he obtained his $\mathrm{PhD}$ degree in 2006 with Prof. Erick M. Carreira. In 2006, he joined Prof. Barry M. Trost at Stanford University as a SNF postdoctoral fellow. Since October 2007 he has been professor of organic chemistry at the Ecole Polytechnique Fédérale de Lausanne (EPFL), where he was promoted full professor in 2019. He is a recipient of the ERC starting grant 2013 and consolidator grant 2017, the Werner prize of the Swiss Chemical Society 2014 and the Springer Heterocyclic Chemistry Award 2016.

\section{ACKNOWLEDGMENT}

We thank the Swiss National Science Foundation (grant number 200020_182798) and EPFL for financial support. 


\section{REFERENCES}

(1) De Meijere, A. Bonding Properties of Cyclopropane and their Chemical Consequences. Angew. Chem., Int. Ed. 1979, 18, 809-826.

(2) Marek, I.; Masarwa, A.; Delaye, P.-O.; Leibeling, M., Selective Carbon-Carbon Bond Cleavage for the Stereoselective Synthesis of Acyclic Systems. Angew. Chem., Int. Ed. 2015, 54, 414-429.

(3) Wang, L. J.; Tang, Y. Asymmetric Ring-Opening Reactions of Donor-Acceptor Cyclopropanes and Cyclobutanes. Isr. J. Chem. 2016, $56,463-475$.

(4) For an overview of the reactivity of cyclopropanes in annulation reactions, see: Liu, J.; Liu, R.; Wei, Y.; Shi, M., Recent Developments in Cyclopropane Cycloaddition Reactions. Trends in Chemistry 2019, 1, 779-793.

(5) Reissig, H. U.; Zimmer, R. Donor-Acceptor-Substituted Cyclopropane Derivatives and their Application in Organic Synthesis. Chem. Rev. 2003, 103, 1151-1196.

(6) Yu, M.; Pagenkopf, B. L. Recent Advances in Donor-Acceptor (DA) Cyclopropanes. Tetrahedron 2005, 61, 321-347.

(7) Carson, C. A.; Kerr, M. A. Heterocycles from Cyclopropanes: Applications in Natural Product Synthesis. Chem. Soc. Rev. 2009, 38 , 3051-3060.

(8) De Simone, F.; Waser, J. Cyclization and Cycloaddition Reactions of Cyclopropyl Carbonyls and Imines. Synthesis 2009, 3353-3374.

(9) Cavitt, M. A.; Phun, L. H.; France, S. Intramolecular DonorAcceptor Cyclopropane Ring-Opening Cyclizations. Chem. Soc. Rev. 2014, 43, 804-818.

(10) Schneider, T. F.; Kaschel, J.; Werz, D. B. A New Golden Age for Donor-Acceptor Cyclopropanes. Angew. Chem. Int. Ed. 2014, 53, 5504-5523.

(11) Grover, H. K.; Emmett, M. R.; Kerr, M. A. Carbocycles from Donor-Acceptor Cyclopropanes. Org. Biomol. Chem. 2015, 13, 655671

(12) O'Connor, N. R.; Wood, J. L.; Stoltz, B. M. Synthetic Applications and Methodological Developments of Donor-Acceptor Cyclopropanes and Related Compounds. Isr. J. Chem. 2016, 56, 431-444.

(13) Pandey, A. K.; Ghosh, A.; Banerjee, P., Reactivity of DonorAcceptor Cyclopropanes with Saturated and Unsaturated Heterocyclic Compounds. Isr. J. Chem. 2016, 56, 512-521.

(14) Talukdar, R.; Saha, A.; Ghorai, M. K. Domino-Ring OpeningCyclization (DROC) of Donor-Acceptor (DA) Cyclopropanes. Isr. J. Chem. 2016, 56, 445-453.

(15) Reiser, O. Catalytic Conversion of Furans and Pyrroles to Natural Products and Analogues Utilizing Donor-Acceptor Substituted Cyclopropanes as Key Intermediates. Isr. J. Chem. 2016, 56, 531-539. (16) Budynina, E. M.; Ivanov, K. L.; Sorokin, I. D.; Melnikov, M. Y. Ring Opening of Donor-Acceptor Cyclopropanes with NNucleophiles. Synthesis 2017, 49, 3035-3068.

(17) Seiser, T.; Cramer, N. Enantioselective Metal-Catalyzed Activation of Strained Rings. Org. Biomol. Chem. 2009, 7, 2835-2840. (18) Souillart, L.; Parker, E.; Cramer, N. Asymmetric Transformations via $\mathrm{C}-\mathrm{C}$ Bond Cleavage. In $C-C$ Bond Activation, Dong, G., Ed. 2014; Vol. 346, pp 163-193.

(19) Souillart, L.; Cramer, N. Catalytic C-C Bond Activations via Oxidative Addition to Transition Metals. Chem. Rev. 2015, 115, 94109464.

(20) Gao, Y.; Fu, X. F.; Yu, Z. X. Transition Metal-Catalyzed Cycloadditions of Cyclopropanes for the Synthesis of Carbocycles: CC Activation in Cyclopropanes. In C-C Bond Activation, Dong, G., Ed. 2014; Vol. 346, pp 195-231.

(21) de Nanteuil, F.; De Simone, F.; Frei, R.; Benfatti, F.; Serrano, E.; Waser, J. Cyclization and Annulation Reactions of NitrogenSubstituted Cyclopropanes and Cyclobutanes. Chem. Commun. 2014, 50, 10912-10928.

(22) Nikolaev, A.; Orellana, A., Transition-Metal-Catalyzed C-C and $\mathrm{C}-\mathrm{X}$ Bond-Forming Reactions Using Cyclopropanols. Synthesis 2016, 48, 1741-1768.

(23) Fumagalli, G.; Stanton, S.; Bower, J. F. Recent Methodologies That Exploit C-C Single-Bond Cleavage of Strained Ring Systems by
Transition Metal Complexes. Chem. Rev. 2017, 117, 9404-9432. (24) Liu, Y.; Wang, Q. L.; Chen, Z.; Zhou, C. S.; Xiong, B. Q.; Zhang, P. L.; Yang, C. A.; Zhou, Q. Oxidative Radical RingOpening/Cyclization of Cyclopropane Derivatives. Beilstein J. Org. Chem. 2019, 15, 256-278.

(25) Jiao, L.; Yu, Z. X. Vinylcyclopropane Derivatives in TransitionMetal-Catalyzed Cycloadditions for the Synthesis of Carbocyclic Compounds. J. Org. Chem. 2013, 78, 6842-6848.

(26) Ganesh, V.; Chandrasekaran, S. Recent Advances in the Synthesis and Reactivity of Vinylcyclopropanes. Synthesis 2016, 48, 4347-4380. (27) Meazza, M.; Guo, H.; Rios, R. Synthetic Applications of Vinyl Cyclopropane Opening. Org. Biomol. Chem. 2017, 15, 2479-2490.

(28) Brownsey, D. K.; Gorobets, E.; Derksen, D. J. Beyond Geminal Diesters: Increasing the Scope of Metal-Mediated Vinylcyclopropane Annulations while Decreasing Pre-Activation. Org. Biomol. Chem. 2018, 16, 3506-3523.

(29) Nakamura, I.; Yamamoto, Y. Transition Metal-Catalyzed Reactions of Methylenecyclopropanes. Adv. Synth. Catal. 2002, 344 111-129.

(30) Brandi, A.; Cicchi, S.; Cordero, F. M.; Goti, A. Heterocycles from Alkylidenecyclopropanes. Chem. Rev. 2003, 103, 1213-1269.

(31) Brandi, A.; Cicchi, S.; Cordero, F. M.; Goti, A. Progress in the Synthesis and Transformations of Alkylidenecyclopropanes and Alkylidenecyclobutanes. Chem. Rev. 2014, 114, 7317-7420.

(32) Yu, L.; Liu, M. X.; Chen, F. L.; Xu, Q. Heterocycles from Methylenecyclopropanes. Org. Biomol. Chem. 2015, 13, 8379-8392.

(33) Yu, L. Z.; Shi, M. The Construction of Molecular Complexity from Functionalized Alkylidenecyclopropanes (FACPs). Chem. Eur. J. 2019, 25, 7591-7606.

(34) Cao, H. G.; Chen, F. L.; Su, C. L.; Yu, L. Construction of Carbocycles from Methylenecyclopropanes. Adv. Synth. Catal. 2020, $362,438-461$

(35) Shi, M.; Shao, L. X.; Lu, J. M.; Wei, Y.; Mizuno, K.; Maeda, H. Chemistry of Vinylidenecyclopropanes. Chem. Rev. 2010, 110, 58835913.

(36) Yang, S.; Shi, M. Recent Advances in Transition-MetalCatalyzed/Mediated Transformations of Vinylidenecyclopropanes. Acc. Chem. Res. 2018, 51, 1667-1680.

(37) For a rare example of enantioselective metathesis on cyclopropenes, see: Giudici, R. E.; Hoveyda A. H. Directed Catalytic Asymmetric Olefin Metathesis. Selectivity Control by Enoate and Ynoate Groups in Ru-Catalyzed Asymmetric Ring-Opening/CrossMetathesis. J. Am. Chem. Soc. 2007, 129, 3824-3825.

(38) Parsons, A. T.; Johnson, J. S. Catalytic Enantioselective Synthesis of Tetrahydrofurans: A Dynamic Kinetic Asymmetric [3+2] Cycloaddition of Racemic Cyclopropanes and Aldehydes. J. Am. Chem. Soc. 2009, 131, 3122-3123.

(39) Campbell, M. J.; Johnson, J. S.; Parsons, A. T.; Pohlhaus, P. D.; Sanders, S. D. Complexity-Building Annulations of Strained Cycloalkanes and $\mathrm{C}=\mathrm{O} \pi$ Bonds. J. Org. Chem. 2010, 75, 6317-6325. (40) Parsons, A. T.; Smith, A. G.; Neel, A. J.; Johnson, J. S. Dynamic Kinetic Asymmetric Synthesis of Substituted Pyrrolidines from Racemic Cyclopropanes and Aldimines: Reaction Development and Mechanistic Insights. J. Am. Chem. Soc. 2010, 132, 9688-9692.

(41) Carson, C. A.; Kerr, M. A. Diastereoselective Synthesis of Pyrrolidines via the $\mathrm{Yb}(\mathrm{OTf})_{3}$ Catalysed Three-Component Reaction of Aldehyde, Amines, and 1,1-Cyclopropanediesters. J. Org. Chem. 2005, 70, 8242-8244.

(42) Kang, Y.-B.; Tang, Y.; Sun, X.-L. Scandium Triflate Catalyzed Cycloaddition of Imines with 1,1-Cyclopropanediesters: Efficient and Diastereoselective Synthesis of Multisubstituted Pyrrolines. Org. Biomol. Chem. 2006, 4, 299-301.

(43) Xu, H.; Qu, J.-P.; Liao, S.; Xiong, H.; Tang, Y. Highly Enantioselective [3+2] Annulation of Cyclic Enol Silyl Ethers With Donor-Acceptor Cyclopropanes: Accessing 3aHydroxy[n.3.0]Carbobicycles. Angew. Chem. Int. Ed. 2013, 52, 40044007. 
(44) de Nanteuil, F.; Serrano, E.; Perrotta, D.; Waser, J. Dynamic Kinetic Asymmetric $[3+2]$ Annulation Reactions of Aminocyclopropanes. J. Am. Chem. Soc. 2014, 136, 6239-6242.

(45) Xiong, H.; Xu, H.; Liao, S.; Xie, Z.; Tang, Y. Copper-Catalyzed Highly Enantioselective Cyclopentannulation of Indoles with DonorAcceptor Cyclopropanes. J. Am. Chem. Soc. 2013, 135, 7851-7854. (46) Wang, D. C.; Xie, M. S.; Guo, H. M.; Qu, G. R.; Zhang, M. C.; You, S. L. Enantioselective Dearomative [3+2] Cycloaddition Reactions of Benzothiazoles. Angew. Chem. Int. Ed. 2016, 55, 1411114115.

(47) Zhang, M. C.; Wang, D. C.; Xie, M. S.; Qu, G. R.; Guo, H. M.; You, S. L. Cu-catalyzed Asymmetric Dearomative $[3+2]$ Cycloaddition Reaction of Benzazoles with Aminocyclopropanes. Chem 2019, 5, 156-167.

(48) Hao, E. J.; Fu, D. D.; Wang, D. C.; Zhang, T.; Qu, G. R.; Li, G. X.; Lan, Y.; Guo, H. M. Chemoselective Asymmetric Dearomative [3 + 2] Cycloaddition Reactions of Purines with Aminocyclopropanes. Org. Chem. Front. 2019, 6, 863-867.

(49) Verma, K.; Banerjee, P. Lewis Acid-Catalyzed [3+2] Cycloaddition of Donor-Acceptor Cyclopropanes and Enamines: Enantioselective Synthesis of Nitrogen-Functionalized Cyclopentane Derivatives. Adv. Synth. Catal. 2016, 358, 2053-2058.

(50) Blom, J.; Vidal-Albalat, A.; Jørgensen, J.; Barløse, C. L.; Jessen, K. S.; Iversen, M. V.; Jørgensen, K. A. Directing the Activation of Donor-Acceptor Cyclopropanes Towards Stereoselective 1,3-Dipolar Cycloaddition Reactions by Brønsted Base Catalysis. Angew. Chem. Int. Ed. 2017, 56, 11831-11835.

(51) Candish, L.; Forsyth, C. M.; Lupton, D. W. N-tert-Butyl Triazolylidenes: Catalysts for the Enantioselective (3+2) Annulation of $\alpha, \beta$-Unsaturated Acyl Azoliums. Angew. Chem. Int. Ed. 2013, 52, 9149-9152

(52) Kang, Y.-B.; Sun, X.-L.; Tang, Y. Highly Enantioselective and Diastereoselective Cycloaddition of Cyclopropanes with Nitrones and Its Application in the Kinetic Resolution of 2-Substituted Cyclopropane-1,1-dicarboxylates. Angew. Chem. Int. Ed. 2007, 46, 3918-3921.

(53) Zhou, J.; Tang. Y. Sidearm Effect: Improvement of the Enantiomeric Excess in the Asymmetric Michael Addition of Indoles to Alkylidene Malonates. J. Am. Chem. Soc. 2002, 124, 9030-9031.

(54) Xu, P. W.; Liu, J. K.; Shen, L.; Cao, Z. Y.; Zhao, X. L.; Yan, J.; Zhou, J. Diastereo- and Enantioselective [3+3] Cycloaddition of Spirocyclopropyl Oxindoles Using Both Aldonitrones and Ketonitrones. Nat. Commun. 2017, 8.

(55) Zhou, Y.-Y.; Li, J.; Ling, L.; Liao, S.-H.; Sun, X.-L.; Li, Y.-X.; Wang, L.-J.; Tang, Y. Highly Enantioselective [3+3] Cycloaddition of Aromatic Azomethine Imines with Cyclopropanes Directed by $\pi-\pi$ Stacking Interactions. Angew. Chem. Int. Ed. 2013, 52, 1452-1456.

(56) Liu, Q.-J.; Yan, W.-G.; Wang, L.; Zhang, X. P.; Tang, Y. One-Pot Catalytic Asymmetric Synthesis of Tetrahydrocarbazoles. Org. Lett. 2015, 17, 4014-4017.

(57) Fu, X.; Lin, L.; Xia, Y.; Zhou, P.; Liu, X.; Feng, X. Catalytic Asymmetric [3+3] Annulation of Cyclopropanes with Mercaptoacetaldehyde. Org. Biomol. Chem. 2016, 14, 5914-5917.

(58) Xu, H.; Hu, J.-L.; Wang, L.; Liao, S.; Tang, Y. Asymmetric Annulation of Donor-Acceptor Cyclopropanes with Dienes. J. Am. Chem. Soc. 2015, 137, 8006-8009.

(59) Prieto, L.; Sánchez-Díez, E.; Uria, U. ; Reyes, E.; Carrillo, L.; Vicario, J. L. Catalytic Generation of Donor-Acceptor Cyclopropanes under N-Heterocyclic Carbene Activation and their Stereoselective Reaction with Alkylideneoxindoles. Adv. Synth. Catal. 2017, 359, 1678-1683.

(60) Halskov, K. S.; Kniep, F.; Lauridsen, V. H.; Iversen, E. H.; Donslund, B. S.; Joergensen, K. A. Organocatalytic EnamineActivation of Cyclopropanes for Highly Stereoselective Formation of Cyclobutanes. J. Am. Chem. Soc. 2015, 137, 1685-1691.

(61) Zhou, Y.-Y.; Wang, L.-J.; Li, J.; Sun, X.-L.; Tang, Y. Side-ArmPromoted Highly Enantioselective Ring-Opening Reactions and Kinetic Resolution of Donor-Acceptor Cyclopropanes with Amines. $J$. Am. Chem. Soc. 2012, 134, 9066-9069.
(62) Kang, Q.; Wang, L.; Zheng, Z.; Li, J.; Tang, Y. Sidearm as a Control in the Asymmetric Ring Opening Reaction of Donor-Acceptor Cyclopropane. Chin. J. Chem . 2014, 32, 669-672.

(63) Luo, W.; Sun, Z.; Fernando, E. H. N.; Nesterov, V. N.; Cundari, T. R.; Wang, H. Asymmetric Ring-Opening of Donor-Acceptor Cyclopropanes with Primary Arylamines Catalyzed by a Chiral Heterobimetallic Catalyst. ACS Catal. 2019, 9, 8285-8293.

(64) Kang, Q.-K.; Wang, L.; Liu, Q.-J.; Li, J.-F.; Tang, Y. Asymmetric $\mathrm{H}_{2} \mathrm{O}-$ Nucleophilic Ring Opening of D-A Cyclopropanes: Catalyst Serves as a Source of Water. J. Am. Chem. Soc. 2015, 137, 1459414597.

(65) Xia, Y.; Lin, L.; Chang, F.; Fu, X.; Liu, X.; Feng, X. Asymmetric Ring-Opening of Cyclopropyl Ketones with Thiol, Alcohol, and Carboxylic Acid Nucleophiles Catalyzed by a Chiral N,N'-DioxideScandium(III) Complex. Angew. Chem. Int. Ed. 2015, 54, 1374813752.

(66) Wales, S. M.; Walker, M. M.; Johnson, J. S. Asymmetric Synthesis of Indole Homo-Michael Adducts via Dynamic Kinetic Friedel-Crafts Alkylation with Cyclopropanes. Org. Lett. 2013, 15, 2558-2561.

(67) Chang, F.; Lin, L.; Xia, Y.; Zhang, H.; Dong, S.; Liu, X.; Feng, X. Chiral N,N'-Dioxide/ScIII Complex-Catalyzed Asymmetric RingOpening Reaction of Cyclopropyl Ketones with Indoles. Adv. Synth Catal. 2018, 360, 2608-2612.

(68) Xia, Y.; Chang, F.; Lin, L.; Xu, Y.; Liu, X.; Feng, X. Asymmetric Ring-Opening of Cyclopropyl Ketones with $\beta$-Naphthols Catalyzed by a Chiral: N, N'-Dioxide-Scandium(III) Complex. Org. Chem. Front. 2018, 5, 1293-1296.

(69) Perrotta, D.; Wang, M.-M.; Waser, J. Lewis Acid Catalyzed Enantioselective Desymmetrization of Donor-Acceptor mesoDiaminocyclopropanes. Angew. Chem. Int. Ed. 2018, 57, 5120-5123.

(70) Zhu, M.; Wang, D. C.; Xie, M. S.; Qu, G. R.; Guo, H. M. Enantioselective Friedel-Crafts Alkylation Reactions of $\beta$-Naphthols with Donor-Acceptor Aminocyclopropanes. Chem. Eur. J. 2018, 24 $15512-15516$

(71) Dickmeiss, G.; De Sio, V.; Udmark, J.; Poulsen, T. B.; Marcos, V.; Jørgensen, K. A. Organocatalytic Asymmetric DesymmetrizationFragmentation of Cyclic Ketones. Angew. Chem. Int. Ed. 2009, 48, 6650-6653.

(72) Cloke, J.B. The Formation of Pyrrolidines from gammaChloropropyl and Cyclopropyl Ketimines. J. Am. Chem. Soc. 1929, 51, 1174-1187.

(73) Wilson, C.L. Reactions of Furan Compounds. VII. Thermal Interconversion of 2,3-dihydrofuran and Cyclopropanecarboxaldehyde. J. Am. Chem. Soc. 1947, 69, 3002-3004. (74) Ortega, A.; Manzano, R.; Uria, U.; Carrillo, L.; Reyes, E.; Tejero, T.; Merino, P.; Vicario, J. L. Catalytic Enantioselective Cloke-Wilson Rearrangement. Angew. Chem. Int. Ed. 2018, 57, 8225-8229.

(75) Xia, Y.; Liu, X.; Zheng, H.; Lin, L.; Feng, X. Asymmetric Synthesis of 2,3-Dihydropyrroles by Ring-Opening/ Cyclization of Cyclopropyl Ketones Using Primary Amines. Angew. Chem. Int. Ed. 2015, 54, 227-230

(76) Xia, Y.; Lin, L.; Chang, F.; Liao, Y.; Liu, X.; Feng, X. Asymmetric Ring Opening/Cyclization/Retro-Mannich Reaction of Cyclopropyl Ketones with Aryl 1,2-Diamines for the Synthesis of Benzimidazole Derivatives. Angew. Chem. Int. Ed. 2016, 55, 12228-12232.

(77) Sanchez-Diez, E.; Vesga, D. L.; Reyes, E.; Uria, U.; Carrillo, L.; Vicario, J. L. Organocatalytically Generated Donor-Acceptor Cyclopropanes in Domino Reactions. One-Step Enantioselective Synthesis of Pyrrolo[1,2-a]quinolines. Org. Lett. 2016, 18, 1270-1273. (78) $\mathrm{Su}, \mathrm{Y}$.; Tu, Y.-Q.; Gu, P. Preparation of Enantioenriched $\gamma$ Substituted Lactones via Asymmetric Transfer Hydrogenation of $\beta$ Azidocyclopropane Carboxylates Using the Ru-TsDPEN Complex. Org. Lett. 2014, 16, 4204-4207.

(79) Sibi, M. P.; Ma, Z.; Jasperse, C. P. Enantioselective Addition of Nitrones to Activated Cyclopropanes. J. Am. Chem. Soc. 2005, 127, 5764-5765.

(80) Kanemasa, S.; Oderaotoshi, Y.; Tanaka, J.; Wada, E. Highly Endoand Enantioselective Asymmetric Nitrone Cycloadditions Catalysed by the Aqua Complex of 4,6-Dibenzofurandiyl-2,2'-bis(4-phenyl- 
oxazoline)-Nickel(II) Perchlorate. Transition Structure Based on Dramatic Effect of MS 4A on Selectivities. J. Am. Chem. Soc. 1998, $120,12355-12356$.

(81) Liu, Q. S.; Wang, D. Y.; Yang, Z. J.; Luan, Y. X.; Yang, J. F.; Li, J. F.; Pu, Y. G.; Ye, M. Ni-Al Bimetallic Catalyzed Enantioselective Cycloaddition of Cyclopropyl Carboxamide with Alkyne. J. Am. Chem. Soc. 2017, 139, 18150-18153.

(82) Amador, A. G.; Sherbrook, E. M.; Yoon, T. P. Enantioselective Photocatalytic [3 + 2] Cycloadditions of Aryl Cyclopropyl Ketones. J. Am. Chem. Soc. 2016, 138, 4722-4725.

(83) Lu, Z.; Shen, M.; Yoon, T.P. [3 + 2] Cycloadditions of Aryl Cyclopropyl Ketones by Visible Light Photocatalysis. J. Am. Chem. Soc. 2011, 133, 1162-1164.

(84) Huang, X.; Lin, J.; Shen, T.; Harms, K.; Marchini, M.; Ceroni, P.; Meggers, E. Asymmetric [3+2] Photocycloadditions of Cyclopropanes with Alkenes or Alkynes through Visible-Light Excitation of Catalyst-Bound Substrates. Angew. Chem. Int. Ed. 2018, $57,5454-5458$

(85) Hao, W.; Harenberg, J. H.; Wu, X.; MacMillan, S. N.; Lin, S. Diastereo- and Enantioselective Formal [3 + 2] Cycloaddition of Cyclopropyl Ketones and Alkenes via Ti-Catalyzed Radical Redox Relay. J. Am. Chem. Soc. 2018, 140, 3514-3517.

(86) Hao, W.; Wu, X.; Sun, J. Z.; Siu, J. C.; MacMillan, S. N.; Lin, S. Radical Redox-Relay Catalysis: Formal [3 + 2] Cycloaddition of NAcylaziridines and Alkenes. J. Am. Chem. Soc. 2017, 139, 1214112144.

(87) Troxler, T.; Scheffold, R. Asymmetric Catalysis by Vitamin B12: The Isomerization of Achiral Cyclopropanes to Optically Active Olefins. Helv. Chim. Acta 1994, 77, 1193-1202.

(88) Müller, P.; Riegert, D. Desymmetrization of Spiro-Activated Meso-Cyclopropanes via Nucleophilic Substitution. Tetrahedron 2005, 61, 4373-4379.

(89) Sparr, C.; Gilmour, R. Cyclopropyl Iminium Activation: Reactivity Umpolung in Enantioselective Organocatalytic Reaction Design. Angew. Chem. Int. Ed. 2011, 50, 8391-8395.

(90) Wallbaum, J.; Garve, L. K. B.; Jones, P. G.; Werz, D. B. RingOpening Regio-, Diastereo-, and Enantioselective 1,3Chlorochalcogenation of Cyclopropyl Carbaldehydes. Chem. Eur. J. 2016, 22, 18756-18759.

(91) Díaz, E.; Reyes, E.; Uria, U.; Carrillo, L.; Tejero, T.; Merino, P.; Vicario, J. L. Carboxylates as Nucleophiles in the Enantioselective Ring-Opening of Formylcyclopropanes under Iminium Ion Catalysis. Chem. Eur. J. 2018, 24, 8764-8768.

(92) Woźniak, Ł.; Magagnano, G.; Melchiorre, P. Enantioselective Photochemical Organocascade Catalysis. Angew. Chem. Int. Ed. 2018, 57, 1068-1072.

(93) Yang, S.; Wang, L.; Zhang, H.; Liu, C.; Zhang, L.; Wang, X.; Zhang, G.; Li, Y.; Zhang, Q. Copper-Catalyzed Asymmetric Aminocyanation of Arylcyclopropanes for Synthesis of $\gamma$-Amino Nitriles. ACS Catal. 2019, 9, 716-721.

(94) Banik, S. M.; Mennie, K. M.; Jacobsen, E. N. Catalytic 1,3Difunctionalization via Oxidative $\mathrm{C}-\mathrm{C}$ Bond Activation. J. Am. Chem. Soc. 2017, 139, 9152-9155.

(95) Wang, M.-M.; Waser, J. 1,3-Difunctionalization of Aminocyclopropanes via Dielectrophilic Intermediates. Angew. Chem. Int. Ed. 2019, 58, 13880-13884.

(96) Yang, J.; Sekiguchi, Y.; Yoshikai, N. Cobalt-Catalyzed Enantioselective and Chemodivergent Addition of Cyclopropanols to Oxabicyclic Alkenes. ACS Catal. 2019, 9, 5638-5644.

(97) Shimizu, I.; Ohashi, Y.; Tsuji, J. Palladium-Catalyzed [3+2] Cycloaddition Reaction of Vinylcyclopropanes with $\alpha, \beta$-Unsaturated Esters or Ketones. Tetrahedron Lett. 1985, 26, 3825-3828.

(98) Trost, B. M.; Morris, P. J. Palladium-Catalyzed Diastereo- and Enantioselective Synthesis of Substituted Cyclopentanes through a Dynamic Kinetic Asymmetric Formal [3+2]-Cycloaddition of Vinyl Cyclopropanes and Alkylidene Azlactones. Angew. Chem. Int. Ed. 2011, 50, 6167-6170.

(99) Trost, B. M.; Morris, P. J.; Sprague, S. J. Palladium-Catalyzed Diastereo- and Enantioselective Formal [3+2]-Cycloadditions of
Substituted Vinylcyclopropanes. J. Am. Chem. Soc. 2012, 134, 17823 17831.

(100) Mei, L. Y.; Wei, Y.; Xu, Q.; Shi, M. Palladium-Catalyzed Asymmetric Formal [3+2] Cycloaddition of Vinyl Cyclopropanes and $\beta, \gamma$-Unsaturated $\alpha$-Keto Esters: An Effective Route to Highly Functionalized Cyclopentanes. Organometallics 2012, 31, 7591-7599. (101) Xie, M. S.; Wang, Y.; Li, J. P.; Du, C.; Zhang, Y. Y.; Hao, E. J.; Zhang, Y. M.; Qu, G. R.; Guo, H. M. A Straightforward Entry to Chiral Carbocyclic Nucleoside Analogues via the Enantioselective [3+2] Cycloaddition of $\alpha$-Nucleobase Substituted Acrylates. Chem. Commun. 2015, 51, 12451-12454.

(102) Li, W. K.; Liu, Z. S.; He, L.; Kang, T. R.; Liu, Q. Z. Enantioselective Cycloadditions of Vinyl Cyclopropanes and Nitroolefins for Functionally and Optically Enriched Nitrocyclopentanes. Asian J. Org. Chem. 2015, 4, 28-32.

(103) Wei, F.; Ren, C. L.; Wang, D.; Liu, L. Highly Enantioselective [3+2] Cycloaddition of Vinylcyclopropane with Nitroalkenes Catalyzed by Palladium(0) with a Chiral Bis(Tert-Amine) Ligand. Chem. Eur. J. 2015, 21, 2335-2338.

(104) Liu, Z. S.; Li, W. K.; Kang, T. R.; He, L.; Liu, Q. Z. PalladiumCatalyzed Asymmetric Cycloadditions of Vinylcyclopropanes and in Situ Formed Unsaturated Imines: Construction of Structurally and Optically Enriched Spiroindolenines. Org. Lett. 2015, 17, 150-153.

(105) Zhou, Q.; Chen, B.; Huang, X. B.; Zeng, Y. L.; Chu, W. D.; He, L.; Liu, Q. Z. Palladium-Catalyzed Diastereo- and Enantioselective Formal [3 + 2] Cycloaddition of Vinyl Cyclopropanes with Cyclic 1Azadienes. Org. Chem. Front. 2019, 6, 1891-1894.

(106) Ma, C.; Huang, Y.; Zhao, Y. Stereoselective 1,6-Conjugate Addition/Annulation of Para-Quinone Methides with Vinyl Epoxides/Cyclopropanes. ACS Catal. 2016, 6, 6408-6412.

(107) Ding, W. P.; Zhang, G. P.; Jiang, Y. J.; Du, J.; Liu, X. Y.; Chen, D.; Ding, C. H.; Deng, Q. H.; Hou, X. L. Electron-Deficient Alkynes as Dipolarophile in Pd-Catalyzed Enantioselective $(3+2)$ Cycloaddition Reaction with Vinyl Cyclopropanes. Org. Lett. 2019, 21, 6805-6810.

(108) Sun, M.; Zhu, Z. Q.; Gu, L.; Wan, X.; Mei, G. J.; Shi, F. Catalytic Asymmetric Dearomative [3 + 2] Cycloaddition of Electron-Deficient Indoles with All-Carbon 1,3-Dipoles. J. Org. Chem. 2018, 83, 23412348.

(109) Mei, L. Y.; Wei, Y.; Xu, Q.; Shi, M. Diastereo- and Enantioselective Construction of Oxindole-Fused Spirotetrahydrofuran Scaffolds through Palladium-Catalyzed Asymmetric [3+2] Cycloaddition of Vinyl Cyclopropanes and Isatins. Organometallics 2013, 32, 3544-3556.

(110) Wang, Q.; Wang, C.; Shi, W.; Xiao, Y.; Guo, H. Pd-Catalyzed Diastereoselective [3 + 2] Cycloaddition of Vinylcyclopropanes with Sulfamate-Derived Cyclic Imines. Org. Biomol. Chem. 2018, 16, 4881-4887.

(111) Ling, J.; Laugeois, M.; Ratovelomanana-Vidal, V.; Vitale, M. R. Palladium(0)-Catalyzed Diastereoselective $(3+2)$ Cycloadditions of Vinylcyclopropanes with Sulfonyl-Activated Imines. Synlett 2018, 29, 2288-2292.

(112) Huang, X. B.; Li, X. J.; Li, T. T.; Chen, B.; Chu, W. D.; He, L.; Liu, Q. Z. Palladium-Catalyzed Highly Enantioselective Cycloaddition of Vinyl Cyclopropanes with Imines. Org. Lett. 2019, 21, 1713-1716. (113) Cao, B.; Mei, L. Y.; Li, X. G.; Shi, M. Palladium-Catalyzed Asymmetric [3+2] Cycloaddition to Construct 1,3-Indandione and Oxindole-Fused Spiropyrazolidine Scaffolds. RSC Adv. 2015, 5, 92545-92548.

(114) Laugeois, M.; Ponra, S.; Ratovelomanana-Vidal, V.; Michelet, V.; Vitale, M. R. Asymmetric Preparation of Polysubstituted Cyclopentanes by Synergistic Pd(0)/Amine Catalyzed Formal [3+2] Cycloadditions of Vinyl Cyclopropanes with Enals. Chem. Commun. 2016, 52, 5332-5335.

(115) Zhu, H.; Du, P.; Li, J.; Liao, Z.; Liu, G.; Li, H.; Wang, W. Synergistic Chiral Iminium and Palladium Catalysis: Highly Regioand Enantioselective $[3+2]$ Annulation Reaction of 2Vinylcyclopropanes with Enals. Beilstein J. Org. Chem. 2016, 12, 1340-1347. 
(116) Meazza, M.; Rios, R. Synergistic Catalysis: Enantioselective Ring Expansion of Vinyl Cyclopropanes Combining Four Catalytic Cycles for the Synthesis of Highly Substituted Spirocyclopentanes Bearing up to Four Stereocenters. Chem. Eur. J. 2016, 22, 9923-9928. (117) Zhang, K.; Meazza, M.; Izaga, A.; Contamine, C.; Gimeno, M. C.; Herrera, R. P.; Rios, R. Synergistic Catalysis: Asymmetric Synthesis of Cyclopentanes Bearing Four Stereogenic Centers. Synthesis 2017, 49, 167-174.

(118) Kamlar, M.; Franc, M.; Císařová, I.; Gyepes, R.; Veselý, J. Formal [3+2] Cycloaddition of Vinylcyclopropane Azlactones to Enals Using Synergistic Catalysis. Chem. Commun. 2019, 55, 3829-3832.

(119) Halskov, K. S.; Næsborg, L.; Tur, F.; Jørgensen, K. A. Asymmetric [3 3 2] Cycloaddition of Vinylcyclopropanes and $\alpha, \beta$ Unsaturated Aldehydes by Synergistic Palladium and Organocatalysis. Org. Lett. 2016, 18, 2220-2223.

(120) Lin, M.; Kang, G. Y.; Guo, Y. A.; Yu, Z. X. Asymmetric Rh(I)Catalyzed Intramolecular $[3+2]$ Cycloaddition of 1-YneVinylcyclopropanes for Bicyclo[3.3.0] Compounds with a Chiral Quaternary Carbon Stereocenter and Density Functional Theory Study of the Origins of Enantioselectivity. J. Am. Chem. Soc. 2012, 134, 398 405

(121) Hashimoto, T.; Kawamata, Y.; Maruoka, K. An Organic Thiyl Radical Catalyst for Enantioselective Cyclization. Nat. Chem. 2014, 6 , 702-705.

(122) Ryss, J. M.; Turek, A. K.; Miller, S. J. Disulfide-Bridged Peptides That Mediate Enantioselective Cycloadditions through Thiyl Radical Catalysis. Org. Lett. 2018, 20, 1621-1625.

(123) Wender, P. A.; Takahashi, H.; Witulski, B. Transition Metal Catalyzed [5 + 2] Cycloadditions of Vinylcyclopropanes and Alkynes: A Homolog of the Diels-Alder Reaction for the Synthesis of SevenMembered Rings. J. Am. Chem. Soc. 1995, 117, 4720-4721.

(124) Wender, P. A.; Husfeld, C. O.; Langkopf, E.; Love, J. A. First Studies of the Transition of Metal-Catalyzed [5+2] Cycloadditions of Alkenes and Vinylcyclopropanes: Scope and Stereochemistry. J. Am. Chem. Soc. 1998, 120, 1940-1941.

(125) Wender, P. A.; Husfeld, C. O.; Langkopf, E.; Love, J. A.; Pleuss, N. The First Metal-Catalyzed Intramolecular [5+2] Cycloadditions of Vinylcyclopropanes and Alkenes: Scope, Stereochemistry, and Asymmetric Catalysis. Tetrahedron 1998, 54, 7203-7220.

(126) Wender, P. A.; Haustedt, L. O.; Lim, J.; Love, J. A.; Williams, T. J.; Yoon, J. Y. Asymmetric Catalysis of the [5 + 2] Cycloaddition Reaction of Vinylcyclopropanes and $\pi$-Systems. J. Am. Chem. Soc. 2006, 128, 6302-6303.

(127) Shintani, R.; Nakatsu, H.; Takatsu, K.; Hayashi, T. RhodiumCatalyzed Asymmetric [5+2] Cycloaddition of AlkyneVinylcyclopropanes. Chem. Eur. J. 2009, 15, 8692-8694.

(128) Straker, R. N.; Peng, Q.; Mekareeya, A.; Paton, R. S.; Anderson, E. A. Computational Ligand Design in Enantio- and Diastereoselective Ynamide [5+2] Cycloisomerization. Nat. Commun. 2016, 7, 1-9.

(129) Cheng, Q.; Xie, J. H.; Weng, Y. C.; You, S. L. Pd-Catalyzed Dearomatization of Anthranils with Vinylcyclopropanes by [4+3] Cyclization Reaction. Angew. Chem. Int. Ed. 2019, 58, 5739-5743.

(130) Trost, B. M.; Yasukata, T. A Catalytic Wagner-Meerwein Shift. J. Am. Chem. Soc. 2001, 123, 7162-7163.

(131) Kleinbeck, F.; Toste, F. D. Gold(I)-Catalyzed Enantioselective Ring Expansion of Allenylcyclopropanols. J. Am. Chem. Soc. 2009, 131, 9178-9179.

(132) Romanov-Michailidis, F.; Guénée, L.; Alexakis, A. Enantioselective Organocatalytic Fluorination-Induced WagnerMeerwein Rearrangement. Angew. Chem. Int. Ed. 2013, 52, 92669270 .

(133) Romanov-Michailidis, F.; Guénée, L.; Alexakis, A Enantioselective Organocatalytic Iodination-Initiated WagnerMeerwein Rearrangement. Org. Lett. 2013, 15, 5890-5893.

(134) Enantioselective Halogenative Semi-Pinacol Rearrangement: A Stereodivergent Reaction on a Racemic Mixture. Chem. Commun. 2014, 50, 13461-13464.

(135) Romanov-Michailidis, F.; Romanova-Michaelides, M.; Pupier M.; Alexakis, A. Enantioselective Halogenative Semi-Pinacol
Rearrangement: Extension of Substrate Scope and Mechanistic Investigations. Chem. Eur. J. 2015, 21, 5561-5583.

(136) Wu, Z.; Lebœuf, D.; Retailleau, P.; Gandon, V.; Marinetti, A.; Voituriez, A. Enantioselective Gold(i)-Catalyzed Rearrangement of Cyclopropyl-Substituted 1,6-Enynes into 2-OxocyclobutylCyclopentanes. Chem. Commun. 2017, 53, 7026-7029.

(137) Trost, B. M.; Bai, W. J.; Hohn, C.; Bai, Y.; Cregg, J. J. PalladiumCatalyzed Asymmetric Allylic Alkylation of 3-Substituted $1 \mathrm{H}$-Indoles and Tryptophan Derivatives with Vinylcyclopropanes. J. Am. Chem. Soc. 2018, 140, 6710-6717.

(138) In, S. K.; Ngai, M. Y.; Krische, M. J. Enantioselective IridiumCatalyzed Carbonyl Allylation from the Alcohol or Aldehyde Oxidation Level Using Allyl Acetate as an Allyl Metal Surrogate. $J$. Am. Chem. Soc. 2008, 130, 6340-6341.

(139) Kim, I. S.; Ngai, M. Y.; Krische, M. J. Enantioselective IridiumCatalyzed Carbonyl Allylation from the Alcohol or Aldehyde Oxidation Level via Transfer Hydrogenative Coupling of Allyl Acetate: Departure from Chirally Modified Allyl Metal Reagents in Carbonyl Addition. J. Am. Chem. Soc. 2008, 130, 14891-14899.

(140) Moran, J.; Smith, A. G.; Carris, R. M.; Johnson, J. S.; Krische, M. J. Polarity Inversion of Donor-Acceptor Cyclopropanes: Disubstituted $\delta$-Lactones via Enantioselective Iridium Catalysis. J. Am. Chem. Soc. 2011, 133, 18618-18621

(141) Zhang, Z. Q.; Meng, X. Y.; Sheng, J.; Lan, Q.; Wang, X. S. Enantioselective Copper-Catalyzed 1,5-Cyanotrifluoromethylation of Vinylcyclopropanes. Org. Lett. 2019, 21, 8256-8260.

(142) Gulías, M.; Durán, J.; López, F.; Castedo, L.; Mascareñas, J. L. Palladium-Catalyzed [4+3] Intramolecular Cycloaddition of Alkylidenecyclopropanes and Dienes. J. Am. Chem. Soc. 2007, 129, 11026-11027

(143) Mazumder, S.; Shang, D.; Negru, D. E.; Baik, M.-H.; Evans, P. A. Stereoselective Rhodium-Catalysed $[3+2+1]$ Carbocyclization of Alkenylidenecyclopropanes with Carbon Monoxide: Theoretical Evidence for a Trimethylenemethane Metallacycle Intermediate. J. Am. Chem. Soc. 2012, 134, 20569-20572.

(144) Verdugo, F.; Villarimo, L.; Durán, J.; Gulías, M.; Mascareñas, J. L.; López, F. Enantioselective Palladium-Catalyzed [3C + 2C] and [4C $+3 \mathrm{C}]$ Intramolecular Cycloaddition of Alkylidenecyclopropanes. ACS Catal. 2018, 8, 6100-6105.

(145) Taillier, C.; Lautens, M. Enantioselective Catalytic Ring Expansion of Methylenecyclopropane Carboxamides Promoted by a Chiral Magnesium Lewis Acid. Org. Lett. 2007, 9, 591-593.

(146) Nemoto, H.; Ishibashi, H.; Nagamochi, M.; Fukumoto, K. A Concise and Enantioselective Approach to Cyclobutanones by Tandem Asymmetric Epoxidation and Enantiospecific Ring Expansion of Cyclopropylidene Alcohols. An Enantiocontrolled Synthesis of (+)and (-)- $\alpha$-Cuparenones. J. Org. Chem. 1992, 57, 1707-1712.

(147) Fukumoto, K.; Nemoto, H.; Tanabe, T.; Nagamochi, M. An Enantiocontrolled Formal Total Synthesis of (+)-Ipomeamarone, (-)Ngaione, and Their Epimers. Heterocycles 1993, 35, 707.

(148) Nemoto, H.; Shiraki, M.; Nagamochi, M.; Fukumoto, K. A Concise Enantiocontrolled Total Synthesis of (-)- $\alpha$-Bisabolol and (+)4-Epi- $\alpha$-Bisabolol. Tetrahedron Lett. 1993, 34, 4939-4942.

(149) Nemoto, H.; Nagamochi, M.; Ishibashi, H.; Fukumoto, K. A Remarkable Substituent Effect on the Enantioselectivity of Tandem Asymmetric Epoxidation and Enantiospecific Ring Expansion of Cyclopropylidene Alcohols: A New Enantiocontrolled Synthesis of ()-Debromoaplysin and (-)-Aplysin. J. Org. Chem. 1994, 59, 74-79.

(150) Nemoto, H.; Tanabe, T.; Fukumoto, K. An Asymmetric Synthesis of Benzylic Quaternary Carbon Centers. A Formal Total Synthesis of (-)-Mesembrine. Tetrahedron Lett. 1994, 35, 6499-6502. (151) Nemoto, H.; Tanabe, T.; Fukumoto, K. A Highly Enantiocontrolled Strategy for the Synthesis of Benzylic Quaternary Carbon Centers. A Formal Total Synthesis of (-)-Mesembrine. J. Org. Chem. 1995, 60, 6785-6790.

(152) Nemoto, H.; Yoshida, M.; Fukumoto, K.; Ihara, M. A Novel Strategy for the Enantioselective Synthesis of the Steroidal Framework Using Cascade Ring Expansion Reactions of Small Ring SystemsAsymmetric Total Synthesis of (+)-Equilenin. Tetrahedron Lett. 1999 
40, 907-910.

(153) Wang, B.; Shen, Y. M.; Shi, Y. Enantioselective Synthesis of $\gamma$ Aryl- $\gamma$-Butyrolactones by Sequential Asymmetric Epoxidation, Ring Expansion, and Baeyer-Villiger Oxidation. J. Org. Chem. 2006, 71, 9519-9521.

(154) Zheng, H.; Felix, R. J.; Gagné, M. R. Gold-Catalyzed Enantioselective Ring-Expanding Cycloisomerization of Cyclopropylidene Bearing 1,5-Enynes. Org. Lett. 2014, 16, 22722275.

(155) Li, C. L.; Yu, Z. X. Asymmetric Synthesis of Azepine-Fused Cyclobutanes from Yne-Methylenecyclopropanes Involving Cyclopropanation/C-C Cleavage/Wagner-Meerwein Rearrangement and Reaction Mechanism. J. Org. Chem. 2019, 84, 9913-9928.

(156) Miura, T.; Nakamuro, T.; Liang, C. J.; Murakami, M. Synthesis of Trans -Cycloalkenes via Enantioselective Cyclopropanation and Skeletal Rearrangement. J. Am. Chem. Soc. 2014, 136, 15905-15908.

(157) Ohmura, T.; Taniguchi, H.; Kondo, Y.; Suginome, M. PalladiumCatalyzed Asymmetric Silaborative C-C Cleavage of MesoMethylenecyclopropanes. J. Am. Chem. Soc. 2007, 129, 3518-3519.

(158) Ohmura, T.; Taniguchi, H.; Suginome, M. Kinetic Resolution of Racemic 1 -Alkyl-2-Methylenecyclopropanes via Palladium-Catalyzed Silaborative C-C Cleavage. Org. Lett. 2009, 11, 2880-2883.

(159) Akai, Y.; Yamamoto, T.; Nagata, Y.; Ohmura, T.; Suginome, M. Enhanced Catalyst Activity and Enantioselectivity with ChiralitySwitchable Polymer Ligand PQXphos in Pd-Catalyzed Asymmetric Silaborative Cleavage of Meso -Methylenecyclopropanes. J. Am. Chem. Soc. 2012, 134, 11092-11095.

(160) Yamamoto, T.; Murakami, R.; Komatsu, S.; Suginome, M. Chirality-Amplifying, Dynamic Induction of Single-Handed Helix by Chiral Guests to Macromolecular Chiral Catalysts Bearing Boronyl
Pendants as Receptor Sites. J. Am. Chem. Soc. 2018, 140, 3867-3870. (161) Nagata, Y.; Takeda, R.; Suginome, M. Asymmetric Catalysis in Chiral Solvents: Chirality Transfer with Amplification of Homochirality through a Helical Macromolecular Scaffold. ACS Cent. Sci. 2019, 5, 1235-1240.

(162) Yonezawa, Y.; Furuyama, T.; Aratani, T.; Fijinami, S.; Inomata, K.; Ukaji, Y. Desymmetrization of meso-methylenecyclopropanes by a palladium-catalyzed asymmetric ring-opening bis(alkoxycarbonylation) reaction. Tetrahedron Asymmetry, 2014, 936-943.

(163) Li, D.-Y.; Wei, Y.; Marek, I.; Tang, X.-Y.; Shi, M. Gold(i)catalyzed cycloisomerization of vinylidenecyclopropane-enes via carbene or non-carbene processes. Chem. Sci. 2015, 6, 5519-5525.

(164) Li, D.-Y.; Fang, W.; Wei, Y.; Shi, M. C( $\left(\mathrm{sp}^{3}\right)-\mathrm{H}$ Functionalizations Promoted by the Gold Carbene Generated from Vinylidenecyclopropanes. Chem. Eur. J. 2016, 22, 18080-18084.

(165) Yang, S.; Rui, K.-H.; Tang, X.-Y.; Xu, Q.; Shi, M. Rhodium/Silver Synergistic Catalysis in Highly Enantioselective Cycloisomerization/Cross Coupling of Keto-Vinylidenecyclopropanes with Terminal Alkynes. J. Am. Chem. Soc. 2017, 139, 5957-5964.

(166) Yang, S.; Li, Q. Z.; Xu, C.; Xu, Q.; Shi, M. Rhodium-Catalyzed Asymmetric Hydroamination and Hydroindolation of KetoVinylidenecyclopropanes. Chem. Sci. 2018, 9, 5074-5081.

(167) Rui, K. H.; Yang, S.; Wei, Y.; Shi, M. Rh(i)-Catalyzed Stereoselective Intramolecular Cycloaddition Reactions of EneVinylidenecyclopropanes for the Construction of Fused 6,5-Bicyclic Skeletons with a Quaternary All-Carbon Stereocenter. Org. Chem. Front. 2019, 6, 2506-2513. 